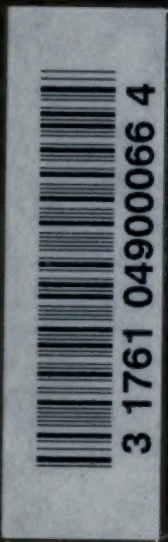

QK
308
C8

UNIV. OF

TORONTO

LIBRARY 

Digitized by the Internet Archive in 2008 with funding from Microsoft Corporation 

Aberdeen University

Studies : No. 54

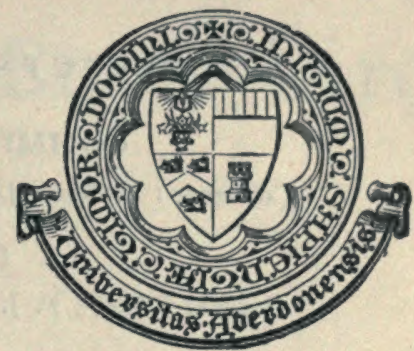

The Flora of Banffshire

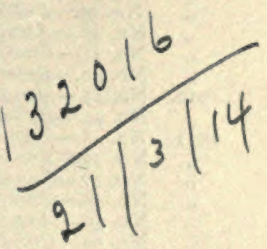




\section{University of Aberdeen.}

\section{COMMITTEE ON PUBLICATIONS.}

Convener: Professor James W. H. TralL, F.R.S., Curator of the Library.

\section{UNIVERSITY STUDIES.}

General Editor: P. J. Anderson, LL.B., Librarian to the University.

1900. No. 1,-Roll of Alumni in Arts of King's College, 1596-1860. P. J. Anderson,

"No. 2.-Records of Old A berdeen, ri57-1891. A. M. Munro, F.S.A. Scot. Vol. I.

"No. 3.-Place Names of West Aberdeenshire. James Macdonald, F.S.A. Scot.

19or. No. 4.-Family of Burnett of Leys. George Burnett, LL.D., Lyon King of Arms.

No. 5.-Records of Invercauld, 1547-1828. Rev. 1. G. Michie, M.A.

1902. No. 6.-Rectorial Addresses in the Universities of Aberdeen, 1835-1900. P. J. Anderson.

$"$ No. 7.-Albemarle Papers, 1746-48. Professor C. S. Terry, M.A.

rgo 3 . No. 8.-House of Gordon. J. M. Bulloch, M.A. Vol. I.

No. 9.-Records of Elgin. William Cramond, LL.D. Vol. I.

rgö4. No. ro.-A vogadro and Dalton. A. N. Meldrum, D.Sc.

" No. 1r.-Records of the Sheriff Court of Aberdeenshire. David Littlejohn, LL.D. Vol. I.

"No. 12.-Proceedings of the Anatomical and Anthropological Society, $1902-04$.

1905. No. 13.-Report on Alcyonaria. Professor J. Axthur Thomson, M.A., and others.

"No. r4.-Researches in Organic Chemistry. Prof. F. R. Japp, F.R.S., and others.

" No. 15.-Meminisse Juvat : with Appendix of Alakeia. Alexander Shewan, M.A

"No. 16,-Blackhalls of that Ilk and Barra. Alexander Morison, M.D.

1906. No. 17,-Records of the Scots Colleges. Vol, I. P. I. Anderson.

" No. 18.-Roll of the Graduates, 1860-1900. Colonel William Johnston, C.B., M.D., LL.D.

" No. 19-Studies in the History of the University. P. J. Anderson and others.

" No. 20. - Studies in the History and Art of the Eastern Provinces of the Roman Empire. Professor Sir W. M. Ramsay, D.C.L., and pupils.

" No. 21.-Studies in Pathology. William Bulloch, M.D., and others.

. No, 22.-Proceedings of the Anatomical and Anthropological Society, $1904-06$.

" No. 23.- Subject Catalogues of the Science Library and the Law Library. P. J. Anderson.

"No. 24.-Records of the Sheriff Court of A berdeenshire. David Littlejohn, LL.D. Vol. II

8907 . No. 25. - Studies on Alcyonarians and Antipatharians. Prof. Thomson, M.A., and others.

" No. 26.-Surgical Instruments in Greek and Roman Times. J. S. Milne, M.A., M.D.

"No. 27--Records of the Sheriff Court of Aberdeenshire. David Littlejohn, LL.D. Vol. III.

" No. 28.-Flosculi Graeci Boreales. Ser. II. Professor John Harrower, M.A.

"No. 29.-Record of the Quatercentenary, 1906. P. J. Anderson.

"No. 30.-House of Gordon. J. M. Bulloch, M.A. Vol. II.

1908. No, $3 \mathrm{r}, \rightarrow$ Miscellany of the New Spalding Club. Vol. II.

" No. 32.-Religious Teachers of Greece. James Adam, Litt.D. (Gifford Lectures, 1904-06.)

". No. 33.-Science and Philosophy of the Organism. Hans Driesch, Ph.D. (Giff. Lect., r907.)

"N No. 34.-Proceedings of the Anatomical and A nthropological Society, 1906-08.

" No. 35.-Records of Elgin. Vol. II. Rev. S. Ree, B.D.

"No. 36.-Pigmentation Survey of School Children. J. F. Tocher, B.Sc.

1909. No. 37.-Science and Philosophy of Organism. Hans Driesch, Ph.D. Vol. II. (Giff. Lect., 1908.)

". No. 38.- Studies on Alcyonarians and Hydroids. Prof. Thomson, M.A., and others. Ser. III.

". No. 39.-Publications of Scottish Clubs. Professor C. S. Terry, M.A.

"No. 40.-A berdeen Friars: Red, Black, White, Grey. P. J. Anderson.

"No. 4r.-Studies on Alcyonarians. Professor Thomson, M.A., and others. Ser. IV.

"No. 42.-Records of Old Abevdeen. A. M. Munro. Vol. II.

19ro. No. 43.-Musa Latina Aberdonensis : Poetae Minores. W. K. Leask, M.A.

"No. 44-Bulletins of the Aberdeen and North of Scotland College of A griculture. Nos. I-14.

rgi1. No. 45.-Records of Inverness. W. Mackay and H. C. Boyd. Vol. I.

" No. 46.-Zoological Studies. Professor Thomson and others. Ser. V.

"No. 47- - Subject Catalogue of the Phillips Library.

. No. 48,-Zoological Studies. Professor Thomson and others. Ser. VI.

$\because$ No. 49.-A nacreontic Poetry of Germany. John Lees. D.Litt.

"No. 50.-Creeds and Confessions of Faith. Professor W. A. Curtis, D.Litt.

"No. 51.-Aberdeen A lumni at Other Universities. Part I. Professor J. Harrower, LL.D.

xg12. No. 52,-Royal Fishery Companies. J. R, Elder, M.A.

" No. 53.-Zoological Studies. Professor Thomson and others. Ser. VII.

"No. 54,-Flora of Banffshire. W. G. Craib, M.A.

"No. 55.-Catalogwe of Anthropological Museum. Professor R. W. Reid, M.D. 


\section{The Flora of Banffshire}

By

William Grant Craib, M.A.

Aberdeen

Printed for the University

MCMXII 


$$
\begin{aligned}
& 9 k \\
& 308 \\
& c 8
\end{aligned}
$$




\section{PRESENTED, 2nd November IgII. \\ PAPER ON "THE FLORA OF BANFFSHIRE" BY WILLIAM G. CRAIB, M.A.}

Moved by the publication of the Flora of Buchan by Professor Trail, of Aberdeen University, by the very meagre information to be got for Banffshire from Professor Dickie's Botanist's Guide, and by the publication of two or three local lists in the Transactions of the Banffshire Field Club, its indefatigable secretary, Mr John Yeats, M.A., conceived the idea that by the systematic co-operation of botanists throughout the county it might be possible to collect such information regarding the distribution of plants in Banffshire as might be worthy of a place in the Club's Transactions. Being responsible for one of the local lists referred to, I was induced by the Secretary and by Mr Grant, the treasurer, to undertake the task with the aid of such botanists and collectors as might be available. Such is the origin of the following botanical survey of the County of Banff.

The area at first contemplated was not the rather artificial geographical area of the county, but the more natural region between the rivers Deveron and Spey, including these river basins. The lack of botanists, however, or more probably their modesty, led to the enforced curtailment of this wider scheme, and only Banffshire proper, with the addition of a few outside parishes on the banks of the rivers named, has been surveyed. The task, though thus restricted, has been no light one. Banffshire, with a seaboard of 32 miles, and a stretch inland in a south-westerly direction of 67 miles, reaching the high elevation of 4296 feet in Ben Mac Dhui, with many other mountains and mountain ranges scarcely inferior in height, bounded by two large rivers and intersected by numerous streams and rills, with all the variations in soil, climate, and rainfall that such a configuration implies affords a field for the botanist of great variety and extent.

The general plan of the work is the same as Professor Trail's Flora of Buchan, to which it may be looked upon as a companion, seeing that three parishes-Gamrie, KingEdward, and Turriff-occur in both, as does also that part of the parish of Alvah lying to the East of Deveron. All the records in the Flora of Buchan are based on Professor Trail's personal observations, but here, where there has been collaboration, the symbols employed are necessarily different, and may be thus explained :-

o denotes old records not verified during the progress of this work.

x . , records by Professor Trail. 
! denotes records by myself.

Dry " that I have seen a dried specimen of the particular plant from that parish. * " records by the folluwing botanists in the parishes against their names :Cullen-Mr James Ritchie, M.A., B.Sc. (records extracted from Professor Trail's record books).

Fordyce and Boyndie-Mr G. D. Thomson, M.A., Kindrought.

Alvah-Miss J. A. Fraser, M.A., Linhead (collection made for the Dickie prize); Mr G. K. Fraser, M.A., Linhead.

Forglen-Mr Stuart, Gardener, Forglen House; Mr G. K. Fraser, M.A., Linhead.

Marnoch-Mr G. K. Fraser, M.A. (collection made for the Dickie prize).

Rothiemay-Miss Jeannie Geddes, M.A.

Cabrach-Mr J. S. Burns, M.A., and Mr J. Yeats, M.A.

Inveravon-Mr Watt, Jun., M.A., B.Sc., Knockando (collection made for the Dickie prize); and Mr Laurence Watt, Glasgow.

Aberlour-Mr John White, M.A., The Orphanage, Aberlour.

Kirkmichael-_-Mr James J. Burgess, M.A., Dyke.

Knockando-Mr C. Watt, Senr., M.A., Knockando.

Ordiquhill-Mr A. Donald, M.A., Ordiquhill Schoolhouse.

Keith-Mr Henry (collection made for Dickie prize, records extracted from Prof. Trail's record books), and Mr G. Petrie Hay, M.A.

Mortlach-Mr Laurence Watt, Glasgow.

To these botanists my best thanks are due for their painstaking work and able assistance. It is impossible here to specify or to attempt to gauge how much the work is indebted to Professor Trail for records in every parish, which he has very generously placed at my disposal. A glance at the tabular statements will at once show that without these records the Flora would have been much less complete.

I am deeply indebted to several other botanists for generous help in the determination of critical species. Mr W. Barclay, Perth, has not only very kindly named all the specimens of the genus Rosa, but, being well acquainted with Banffshire, he has also written up the distribution of that genus from his own personal observation and from specimens forwarded. The Rev. E. F. Linton has determined several consignments of Hieracia, chief among which may be mentioned a large collection from Gamrie, which yielded several novelties for 
Banffshire. All specimens of the genus Rubus which were sent to me, or were collected by me, have been named by Rev. W. M. Rogers.

With regard to the resent lists in the Transactions of the Banffshire Field Club, viz., that of the Boyne district by Mr Thomson, Kindrought, and that of the Banff district by myself, these may be taken to be superseded by the present list. Mr Thomson has kindly forwarded to me notes giving several additions to his previous list, a few corrections, and full notes of the parishes in which the plants were found by him.

The Rev. Dr. W. S. Bruce, of Banff, has also contributed to the Transactions several papers dealing with the Flora of Banffshire; but, unfortunately, these papers are practically useless for the present work, owing either to the localities mentioned being too vague, or to the use of local names of the plants. He records Carex incurva and Senebiera Coronopus for Banff, and Pyrola secunda for Alvah, plants which I have been unable to find in the districts mentioned. There is still another published list dealing with part of the district under review : a list for the Parish of Cairnie, given in Pirie's "Parish of Cairnie" (1906). As this list contains several mistakes, I prefer to omit the records contained in it, and to rely on the records of Prof. Trail for that parish.

The most difficult, though most pleasant, duty remains, to express my heartfelt thanks, however inadequately, to Mr Yeats. To him, the originator and inspirer of this Flora, has fallen the arduous task of maintaining the correspondence with the local workers which such a work involves. This from the beginning of the enterprise he has most ungrudgingly performed, sparing neither valuable time noc advice.

With regard to the old records, it is matter of regret that space does not allow of a complete list, but below is appended a list of the old records cited. So far as possible only the earliest records are given, but as they have been extracted from various sources, and as all the original authorities have not been examined by me, it is possible that there may be mistakes and omissions.

The following is a list of the contractions used for the works cited :-

B.G. ... Dickie's "Botanist's Guide."

N.F. ... Dr Murray's "Northern Flora."

S.A. ... "New Statistical Account of Scotland."

F.B. .. Professor Trail's "Flora of Buchan," including the Supplement.

Ed. ... Tom Edward (communicated by Professor Trail).

M. ... Marshall and Shoolbred, Kirkmichael, Journ. Bot. 1906, pp. 154-161. 


\section{RANUNCULACEF.}

Thaliotrum minus, Linn.-Rathven, B.G.; Oullen, B.G.

Anemone nemorosa, Linn.-King Edward, S.A.; Turriff, F.B.

A. apennina, Linn.-Rathven, 8.A.

Ranunculus aquatilis, Linn.-King Edward, F.B.; Turriff, F.B.

ค. fuitans, Lam,-King Edward, F.B.

A. Lenormandi, F. Sohulte-Alvah, F.B.

P. hederacous, Linn.-Turriff, F.B.

A. sceleratus, Linn.-Alvah, B.G.

ค. Flammula, Linn.-Turriff, F.B.

b. radicans, Nolte-Gamrie, F.B.; King Edward, F.B.; Turriff, F.B.

ค. scoticus, E. S. Marshall-Kirkmichael, M.

R. acris, Linn., b. Friesianus, R. \& F.-Turriff, F.B.

c. Bormanus, (Jord.)-Turriff, F.B.

0. rectus, (Jord.)-Turriff, F.B.

R. bulbosus, Linn.-Rathven, B.G.; Alvah, B.G.; Turriff, Skene; Mortlach, B.A

R. Ficaria, Linn.-King Edward, F.B.; Turriff, F.B.

Caltha radicans, Forster-Kirkmichael, M.

Trollius europæus, Linn.-Alvah, B.G.

\section{BERBERIDACEAE.}

Berberis vulgaris, Linn.-King Edward, F.B.

\section{PAPAVERACEA.}

Papaver Rhceas, Linn.-Alvah, F.B.

P. dubium, Linn.-Grange, 8.A.

Chelidonium majus, Linn.-Mortlach, B.G.

\section{FUMARIACEA.}

Corydalis claviculata, DO.-Mortlach, B.G.

Fumaria caproolata, Linn.-Turriff, F.B.; Grange, B.A.

F. Bastardi, Bor--Gamrie, F.B.; King Edward, F.B.; Turriff, F.B.

F. officinalis, Linn,-Turriff, F.B.; Grange, 8.A.

\section{CRUCIFERE.}

Barbarea vulgaris, Ait.-King Edward, F.B.

A rabis potraa, Linn., b. hispida, DO.-Kirkmichael, $\mathbb{M}$.

Cardamine amara, Linn.-Turriff, F.B.; Mortlaoh, B.G.

c. pratensis, Linn.-Turriff, F.B.

b. dentata, (Bohultes)-Kirkmiohael, M.

C. hirsuta, Linn.-Turriff, F.B.; Grange, 8.A.

C. fexuosa, Linn.-King Edward, F.B.; Turriff, F.B

Draba incana, Linn.-Boyndie, Ed.; Kirkmichael, M.

D. rupestris, Br.-Kirkmiohael, B.G.

Erophila vorna, E. Mey.-Turrie, F.B.; Grange, 8.A. 
Cochlearia alpina, Wats.-Kirkmichael, M.

Sisymbrium Thalianum, Gay-Banff, Ed.; Alvah, B.G.; King Edward, F.B., Kirkmichael, M.

S. officinale, Soop.-Grange, B.A.

s. Alliaria, Scop.-Mortlach, B.G.

Brassica alba, Boiss.-Gamrie, F.B.

Coronopus procumbens, Gilib.-Gamrie, F.B.; Banff, Bruce (v. Introd.)

Lepidium sativum, Linn,-Rathven, Ed.

L. heterophyllum, Benth., b. canescens, Gren. \& Godr.-Mortlach, B.G.

Thlaspi arvense, Linn.-Rathven, Ed.; Gamrie, F.B.; Alvah, B.G.; Mortlach, B.G.

Iberis amara, Linn.-Oullen, Ed.

\section{RESEDACEA.}

neseda Luteola, Linn.-Rathren, B.G.; Mortlach, B.G.

R. odorata, Linn.-Oullen, Ed.

\section{Cistacefe.}

Holianthemum Chamæcistus, Mill.-Rathven, B.G.; Alvah, S.A.; Turriff, F.B.; Mortlach, B.G.

\section{VIOLACEAE.}

Viola palustris, Linn.-King Edward, F.B.; Turriff, F.B.

v. tricolor, Linn.-King Edward, F.B.

v. arvensis, Murr.-Turriff, F.B.

v. Iutea, Huds, \& f. amoena, (Symons)-Kirkmichael, $\mathbf{M}$.

v. Cornuta, Linn.-Turrifi, F.B.

\section{Polygalacefe.}

Polygala vuigaris, Linn.-Kirkmichael, M.

P. serpyllacea, Weihe-King Edward, F.B.

\section{GARYOPHYLLACEAE.}

Silene latifolia, Rendle \& Britten-Mortlach, B.G.

S. anglica, Linn.-Rathven, Ed.

s. dichotoma, Ehrh.-Kirkmiohael, $\mathbb{M}$.

Lyohnis alba, Mill.-Mortlach, B.G.

L. Githago, Bcop.-Gamrie, F.B.; Turrifi, F.B.; Grange, S.A.

Corastium semidecandrum, Linn.-Alvah, B.G.; Grange, S.A.

C. viscosum, Linn., b. apetalum, Dum.-Alvah, F.B.; King Edward, F.B.; Turriff, F.B.

C. alpinum, Linn., b. pubescens, Syme-Kirkmichael, M.

c. arvense, Linn.-Turriff, B.A.; Mortlach, B.G.

C. cerastoides, Britton-Kirkmichael, B.G.

Stellaria nemorum, Linn.-Alvah, F.B.; Turriff, S.A.

S. Holostea, Linn.-King Edward, F.B.

S. uliginosa, Murr.-King Edward, F.B.; Turriff, F.B.

Arenaria trinervia, Linn.-Alvah, B.G.

A. leptoclados, Guss.-Kirkmichael, $\mathrm{M}$.

Sagina ciliata, Fr.-Gamrie, F.B.

s. saginoides, Dalla Torro-Kirkmiohael, M.

s. subulata, Presl-Kirkmichael, M.; Aberlour, B.G.

s. nodosa, Fenzl.-Alvah, B.G.; Mortlaoh, B.G.

Spergularia rubra, Pers,-Grange, \$.A. 


\section{PORTULACACEA.}

Montia fontana, Linn.-Turriff, F.B.

\section{HYPERICACEA.}

Hypericum perforatum, Linn.-Alvah, S.A.; Grange, 8.A.

H. quadratum, Btokes-Alvah, B.G.; Turriff, F.B.

H. humifusum, Linn.-Gamrie, F.B.; Mortlach, B.G.

н. hirsutum, Linn.-Kirkmiohael, M.

\section{MALVACE EE.}

Lavatera arborea, Linn.-Rathven, Ed.

Malva moschata, Linn.-Turriff, F.B.; Oairnie, P.S.

M. sylvestris, Linn.-Turriff, F.B.; Mortlach, B.G.

Tilia intermedia-Bellie, 8.A.

\section{TILIACEAE.}

\section{LINAGEAE.}

Radiola linoides, Roth.-Alvah, B.G.

Linum catharticum, Linn.-King Edward, F.B.

\section{GERANIACEAE.}

Ceranium sylvaticum, Linn.-Gamrie, F.B.; King Edward, S.A.

C. pratense, Linn,-Cullen, 8.A.; Gamrie, F.B.; King Edward, 8.A.; Kirkmichael, B.G.

C. dissectum, Linn.-Grange, 8.A.

a. Iucidum, Linn,-Kirkmichael, M.

C. Robertianum, Linn.-Grange, S.A.

b. modestum, (Jord.)-Kirkmichael, M.

Oxalis Acetosella, Linn.-Turriff, F.B.

\section{RHAMNACE E.}

Rhamnus catharticus, Linn.-Alvah, B.G.

\section{LECUMINOSAE.}

Conista anglica, Linn.-King Edward, S.A.; Grange, 8.A.

Ononis repens, Linn.-Alvah, B.G.; Aderlour, Ed.; Mortlach, B.G.

Medicago sativa, Linn.-Rathren, Ed.

m. Iupulina, Linn.-Grange, 8.A.

Molilotus alba, Desr.-Rathyen, Ed.

M. offcinalis, Lam,-Rathven, Ed.; Gamrie, F.B.

Trifolium arvense, Linn.-Boyndie, Ed.; Alvah, B.G.

T. striatum, Linn.-Boyndie, Ed.

T. agrarium, Linn,-Gamrie, F.B.

Anthyllis Vuineraria, Linn,-King Edward, F.B.

Astragalus giyeyphyllos, Linn.-Boyndie, B.G.

Ornithopus perpusillus, Linn.-Alvah, B.G.

Vicia hirsuta, Gray-Alvah, B.G.

v. sativa, Linn.-King Edward, F.B.; Turriff, F.B.

v. angustifolia, Linn.-Alvah, B.G.; King Edward, F.B.; Turriff, F.B.; Grange, B.A.; Mortlaoh, B.G.

V. Iathyroides, Linn.-Aivah, B.G.

Lathyrus montanus, Bernh.-King Edward, F.B.; Turriff, F.B.

b. tenuifolius, Roth.-Turriff, B.A. 


\section{ROSACEAE.}

Prunus spinosa, Linn.-King Edward, F.B.; Turriff, F.B.

P. avium, Linn.-Gamrie, F.B.; Turriff, F.B.

P. Gerasus, Linn.-Alvah, B.G.

P. Padus, Linn.-Turriff, F.B.

Spiraea salicifolia, Linn.-Gamrie, F.B.; King Edward, F.B.

Rubus plicatus, Wh. \& N.-Alvah, F.B.

R. melanoxylon, Muell. \& Wirtg.-Gamrie, F.B.; Turriff, F.B.

R. infestus, Weihe-Gamrie, F.B.; Alvah, F.B.; King Edward, F.B.

R. radula, Weihe-Gamrie, F.B.

R. Koehieri, Wh. \& N.-Gamrie, F.B.

R. corylifolius, Sm.-Alvah, 8.A.; King Edward, F.B.

R. saxatilis, Linn.-Alvah, B.G.; Turriff, F.B.

Goum rivale, Linn.-King Edward, S.A.; Turriff, S.A.

Fragaria vesca, Linn.-Gamrie, F.B.; King Edward, F.B.; Turriff, F.B.; Grange, \$.A.

F. moschata, Ducherne-Alvah, Ed.; King Edward, F.B.

Potentilla sterilis, Garoko-Alvah, B.G.

P. procumbens, Sibth.-Gamrie, F.B.; King Edward, F.B.; Turriff, F.B.

P. palustris, Soop.-King Edward, F.B.; Turriff, F.B.

Alchomilla vulgaris, Linn., a. pratensis, Pohl-King Edward, F.B.; Turriff, F.B.

b. alpestris, Pohl-Turriff, F.B.

c. filicaulis, (Buser)-Kirkmichael, M.

A. alpina, Linn.-Alvah, B.G.; King Edward, S.A.; Grange, N.F.; Keith, B.G.; Mortlach, B.G.

A. arvensis, Scop.-Turriff, F.B.

Rosa pimpinellifolia, Linn.-Kirkmichael, M.

f. spinosissima, Linn.-Alvah, B.G.; Grange, S.A.; Mortlach, B.G.

R. tomentosa, Sm.-Kirkmichael, M.; Grange, 8.A.

R. canina, Linn., agg.-Alvah, B.G.; Mortlach, B.G.

R. glauca, Vill., e. subcristata, (Baker)-Kirkmichael, M.

Agrimonia Eupatoria, Linn.-Rathven, S.A.; Banff, S.A.; Gamrie, S.A.; Alvah, B.G.; Turrıff, F.B.; Inverkeithnie, B.G.

Pyrus Aria, Ehrh.-Gamrie, F.B.; King Edward, F.B.

b. rupicola, Syme-Kirkmichael, M.

\section{SAXIFRAGACEA.}

saxifraga aizoides, Linn.-Alvah, B.G.; King Edward, S.A.; Mortlach, B.G.

S. granulata, Linn.-Rathven, B.G.

8. hypnoides, Linn.-Turriff, F.B.

Chrysosplenium alternifolium, Linn.-Gamrie, S.A.; Turriff, F.B.

Parnassia palustris, Linn.-Mortlaoh, B.G.

Ribos Grossularia, Linn.-Gamrie, F.B.; King Edward, F.B.; Turriff, F.B.

R. alpinum, Linn.-Mortlaoh, B.G.

R. nigrum, Linn.-Alvah, F.B.; King Edward, F.B.

\section{CRAsSulacefe.}

sedum purpuroum, Tausch-Kirlemichael, $M$.

S. villosum, Linn.-Alvah, B.G.; Turriff, S.A.; Mortlach, B.G.

\section{DROSERAGEA.}

Drosera rotundifolia, Linn.-Gamrie, F.B.; King Edward, F.B.

D. anglica, Huds.-King Edward, F.B.; Mortlaoh, Ed. $\times$ rotundifolia-King Edward, F.B. 


\section{HALORAGACEA.}

Hippuris vulgaris, Linn.-Gamrie, 8.A.; Turriff, F.B.; Oairnie, N.F.

Myriophyllum alterniflorum, DO.-King Edward, F.B.; Turriff, F.B.; Kirkmichael, M.

Callitricho verna, Linn., agg.-Turriff, F.B.

C. stagnalis, Scop.-King Edward, F.B.; Turriff, F.B.

C. intermedia, Hoffm.-Gamrie, F.B.; King Edward, F.B.; Turriff, F.B.

\section{ONAGRACEA.}

Epilobium hirsutum, Linn.-Turriff, F.B.

E. angustifolium, Linn.-Kirkmichael, B.G.

E. obscurum, Bohreb,-King Edward, B.A.; Turriff, F.B.

E. parviforum, Bohreb.-Mortlach, B.G.

E. alsinefolium, Vill.-Kirkmiohael, B.G.

E. anagallidifolium, Linn.-Aberlour, B.G.

E. hybrids-Kirkmichael, $\mathbf{M}$.

Circæa alpina, Linn,-Bathven, S.A.; Turriff, 8.A.; Mortlaoh, B.G.

UMBELLIFERA.

Hydrocotyle vulgaris, Linn.-Alvah, S.A.; King Edward, F.B.; Turriff, F.B.

Sanicula ouropæa, Linn.-Gamrie, S.A.; Alvah, B.G.; Mortlach, B.G.

Conium maculatum, Linn.-Grange, B.A.; Mortlach, B.G.

Pimpinolla Saxifraga, Linn.-Alvah, B.G.

Chærophyllum tomulum, Linn.-Alvah, B.G.

Scandix Pecten-Veneris, Linn.-Alvah, B.G.; Mortlach, B.G.

An.hriscus vulgaris, Bernh.-Rathven, Ed.

Meum Athamanticum, Jacq.-Kirkmichael, M.

Peucedanum sativum, Benth. \& Hook, 1.-Gamrie, F.B.

Caucalis Anthriscus, Huds.-Mortlach, B.G.

\section{CAPRIFOLIACEAE.}

Adoxa Moschatollina, Linn.-Rathven, B.G.; Oullen, \$.A.; Boyndie, Ed,; King Edward, B.A.: Mortlach, B.G.

Sambuous Ebulus, Iinn.-Mortlach, B.G.

Linnæa borealis, Linn.--Bellie, S.A.; Rathven, B.G.: King Edward, B.A.; Turriff, S.A; Grango, Ed.; Mortlaoh, B.G.

RUBIACEA.

Calium boreale, Linn.-Aivah, B.G.; Mortlach, B.G.

C. Cruciata, Scop.-Keith, B.G.-Professor Trail has informed me that this is a wrong identifloation, the plant being a species of $\Delta$ sperula.

C. Mollugo, Linn.-Gamrie, F.B.; Alvah, B.G.; Grange, Ed.

C. asperum, Sohreb.-Gamrie, F.B.

C. palustro, Linn.-King Edward, F.B.; Turriff, F.B.

G. uliginosum, Linn.-Alvah, B.G.; Turriff, F.B.; Kirkmichael, M.; Grange, 8.A.

Asperula odorata, Linn.-Bellie, \$.A.; Boyndie, Ed.; Gamrie, F.B.; Alvah, B.G.; Turriff, 8.A.; Kirkmiohael, M.; Mortlaoh, B.G.

Sherardia arvensis, Linn.-Turriff, F.B.; Grange, B.A.

VALERIANAGEAE.

Valeriana sambuelfolla, Mikan,-King Edward, F.B.

V. pyrenaica, Linn.-Gamrie, F.B.

Valerianella olitoria Poll.-Alvah, B.G.; Keith, B.G.

v. dentata, Poll.-Aivah, F.B. 


\section{COMPOSITF.}

Eupatorium cannabinum, Linn.-Gamrie, S.A.

Solidago Virgaurea, Linn.-King Edward, \$.A.

Erigeron aore, Linn.-Kirkmichael, M.

Filago germanica, Linn.-Gamrie, F.B.; Alvah, B.G.

F. minima, Fr.-King Edward, F.B.; Mortla.oh, B.G.

Antennaria dioica, Gaertn.-Mortlach, B.G.

Cnaphalium uliginosum, Lipn.-Turriff, F.B.

G. sylvaticum, Linn.--Turriff, F.B.; Grange, S.A.; Mortlaeh, B.G.

Bidens cernua, Linn.-Alvah, B.G.

Achillea tomentosa, Linn.-Boharm, B.G.

Chrysanthemum segetum, Linn.-Turriff, F.B.; Grange, S.A.

C. Parthenium, Bernh.-Gamrie, F.B.; Turriff, F.B.

Matricaria inodora, Linn.-Turriff, F.B.

Tanacotum vulgare, Linn.--King Edward, F.B.; Turriff, F.B.; Mortlach, B.G.

Artemisia vulgaris, Linn.-King Edward, F.B.; Grange, S.A.

Petasites ovatus, Hill-Gamarie, F.B.; King Edward, F.B.

Doronicum plantagineum, Linn.-Banff, Ed.

Senecio sylvatious, Linn.-Grange, S.A.; Turriff, F.B.

Arctium spp.-Alvah, B.G.; Grange, s.A.

Cnicus heterophyllus, Willd.-Mortlach, B.G.

Saussurea alpina, Linn.-Kirkmichael, $M$.

Centaurea Cyanus, Linn.-Alvah, B.G.; Grange, S.A.; Mortlaoh, B.G.

Cichorium Intybus, Linn.-Inverkeithnie, B.G.

Crepis mollis, Asohers.-Kirkmichael, $M$.

Hieracium Pilosella, Linn., c. nigrescens, Fr., d. concinnatum, F. J. Hanb.; H. anglicum, Fr. \& b. acutifolium, Backh.; H. iricum, Fr.; H. holosericeum, Backh.; H. eximium, Backh., b. tenellum, Backh.; H. gracilentum, Backh.; H. petiolatum, Elfstrand; H. lingulatum, Baokh.; H. chrysanthum, Baokh.; H. nigrescens, Willd., c. gracilifolium, F. J. Hanb.; H. Schmidtii, Tausch, \& c. crinigerum, Fr.; H. argenteum, Fr.; H. Sommerfeltii, Lindeb.; H. pseudonosmoides, Dahlst.; H. silvaticum, Gouan, $\mathbf{f}$. prolixum, Dahlst.; H. variicolor, Dahlst.; H. serratifrons, Almq., b. Stenstroemii, Dahlst.; H. euprepes, F. J. Hanb., c. clivicolum, F. J. Hanb.; H. angustatum, Lindeb.,

b. elatum, Lindeb.; H. corymbosum, Fr.; H. auratum, Fr.-Kirkmichael, M.

H. aurantiacum, Linn.-Turriff, B.G.

H. murorum, Linn.-Gamrie, F.B.

H. cosium, Fr.-Banff, J. Roy (comm. Prof. Trail).

H. vulgatum, Fr.-Gamrie, F.B.; King Edward, F.B.; Turriff, F.B.

H. boreale, Fr.-Alvah, B.G.

Taraxacum paiustre, DO.-Alvah, B.G.

Sonchus oloraceus, Linn.-Turriff, F.B.; Grange, S.A.

S. asper, Hill-Turriff, F.B.

s. arvensis, Linn.-Turriff, F.B.

CAMPANULACEF.

Campanula latifolia, Linn.-Rathven, \$.A.; Oullen, B.G. : Turriff, S.A.

VAGGINIACEE.

Vacoinium Vitis-Idæa, Linn.-Rathven, 8.A.; Cullen, B.G.; Banff, Ed.; Alvah, B.G.; Turriff, F.B.

Oxycoceus quadripotala, Gilib.-Rathven, S.A. 


\section{ERICAGEAE.}

Erica Totralix, Linn。-King Edward, F.B.

Azalea procumbens, Linn.-Mortlach, B.G.

Pyrola modia, Sw.-Alvah, B.G.; Turriff, S.A.; Mortlach, B.G.

P. rotundifolia, LInn.-Turriff, B.G.

P. minor, Linn.-Turriff, 8.A.; Mortlach, B.G.

P. secunda, Linn.-Turriff, 8.A.; Rothiemay, B.G.; Mortlach, B.G.; Alvah, Bruce (v. Introd.)

Monoses unifiora, A. Gray-Rothiemay, B.G.; Mortlach, B.G.

\section{PRimulagefe.}

Primula vulgaris, Hudg.-King Edward, F.B.; Turrifr, F.B.; Grange, S.A.

P. veris, Linn.-Gamrie, B.G.

P. olatior, Jacq.-Alvah, B.G.

Lysimachia nemorum, Linn.-Gamrie, F.B.; King Edward, F.B.; Turrif, 8.A.; Grange. 8.A. Trientalis europæa, Linn.-Cullen, B.G.; Gamrie, F.B.; Turriff, S.A.

Anagallis arvensis, Linn.-Alvah, B.G.

A. tenella, Murr.-Alvah, B.G.; Mortlach, B.G.

\section{APOCYNACEA.}

Vinca minor, Linn.-King Edward, 8.A.

GENTIANACEAE.

Centiana Amarella, Linn.-Boyndie, Ed.

C. baltica, Murb-Gamrie, F.B.

Menyanthes trifoliata, Linn.-Turriff, F.B.; Grange, \$.A.

Nymphoides peltatum, Rendle \& Britten-Turriff, F.B.

BORACINACEA.

Cynoglossum ofncinale, Linn.-Boyndie, B.G.

Symphytum peregrinum, Ledeb.-Gamrie, F.B.: Turriff, F.B.

Anchusa sempervirens, Linn.-Mortlach, B.G.

Borago omoinalis, Linn.-Rathven, B.G.: Oullen, S.A.

Lycopsis arvensis, Linn.-Turriff, F.B.; Grange, \$.A.

Mertensia maritima, Gray-Banff, S.A.

Myosotis cespitosa, Schultz-King Fdward, F.B.; Turriff, F.B.

M. scorpioides, Linn.-Camrie, F.B.; King Edward, F.B.; Turriff, F.B.

b. strigulosa, (Reichb.)-Kirkmiohael, M.

M. eyivatica, Hoff $\mathbf{m}$.-Alvah, F.B.

M. collina, Hoffm.-Banff, B.G.; Gamrie, S.A.; Alvah, B.G.

M. versicolor, Bm.-King Edward, F.B.; Turriff, F.B.

Lithospermum arvense, Linn.-Alvah, B.G.; Mortlach, B.G.

Eohium vulgare, Linn,-Alvah, B.G.: Mortlach, B.G.

\section{SOLANACEF.}

Solanum Duleamara, Linn.-Gamrie, N.F.

s. nigrum, Linn,-Boyndie, Ed.

\section{SCROPHULARIACEE.}

Linaria vuigaris, Mill.-Rathren, Ed.; Gamrie, F.B.; Mortlach, B.G.

Sorophularia nodosa, Linn.-Mortlach, B.G. 
Mimuius guttatus, Linn.-Kirkmichael, $\mathbf{M}$.

Veronica hederæfolia, Linn.-King Edward, F.B.: Grange, S.A.

v. didyma, Ten.-Turriff, F.B.

V. agrestis, Linn.-King Edward, F.B.; Turriff, F.B.

v. Tournefortii, O. Gmel.-Turriff, F.B.

v. arvensis, Linn.-King Edward, F.B.

v. serpyllifolia, Linn., b. tenella, All.-Kirkmichael, M.

v. alpina, Linn.-Kirkmiohael, M.

v. montana, Linn.-Bellie, N.F.; Gamrie, 8.A.

v. seutellata, Linn.-Turriff, F.B.; Mortlach, B.G.

b. hirsuta, Weber,-Alvah, B.G.

v. Anagallis-aquatica, Linn.-King Edward, B.G.

Euphrasia brevipila, Burnat \& Gremli-Gamrie, F.B.; King Edward, F.B.; Turriff, F.B.

E. borealis, Towns.-Gamrie, F.B.; King Edward, F.B.

E. gracilis, Fr.-Gamrie, F.B.; King Edward, F.B.; Turriff, F.B.

E. scottica, Wettst.-Gamrie, F.B.

E. curta, Wettst.-Gamrie, F.B.

Podicularis palustris, Linn.-King Edward, F.B.; Turriff, F.B.

P. syivatica, Linn.-King Edward, F.B.; Turriff, F.B.

Ahinanthus major, Ehrh., c. apterus, Fr.-Kirkmichael, M.

R. borealis, Druce-Kirkmiohael, $M$.

Molampyrum pratense, Linn.-Alvah, B.G.; Turriff, S.A.

d. montanum, Johnst,-Kirkmichael, M.

๑. hians, Druce-Kirkmichael, M.

\section{LENTIBULARIACEA.}

Utrieularia minor, Linn.-Alvah, S.A.; Turriff, F.B.

U. intermedia, Hayne-Turriff, F.B.

Pinguicula vulgaris, Linn.-King Edward, F.B.; Turriff, F.B.

\section{LABIATE.}

Mentha alopecuroides, Hull-Kirkmichael, M.

M. piperita, Linn.-King Edward, F.B.; Turriff, F.B.

M. aquatica, Linn., a. hirsuta, (Huds.)-King Edward, F.B.; Turriff, F.B.; Kirkmichael, M.; Grange, 8.A.

M. arvensis, Linn.-Turriff, F.B.

M. paludosa, Sole-King Edward, F.B.

Thymus Serpyllum, Linn.-Alvah, F.B.; Turrifr, F.B.; Forglen, S.A.

Clinopodium vulgare, Linn.-Kirkmichael, $M$.

Nepeta hederacea, Trev.-Grange, S.A.

Scutellaria galericulata, Linn,-Mortlach, B.G.

Marrubium vuigaro, Linn,-Rothiemay, Ed.

Stachys palustris, Linn.-Gamrie, F.B.; Turriff, F.B.

5. arvensis, Linn.-Alvah, B.G.; King Edward, F.B.; Mortlach, B.G.

Galeopsis speciosa, Mill.-Kirkmichael, M.; Mortlach, B.G.

Lamium amplexicaule, Linn.-Alvah, B.G.; King Edward, F.B.; Turriff, F.B.; Grange, S.A.

L. maculatum, Linn.-Rathven, S.A.; King Edward, F.B.; Turriff, F.B.

L. album, Linn.-King Edward, F.B.

Teucrium Scorodonia, Linn.-Gamrie, F.B.; Turriff, F.B.

Ajuga reptans, Linn.-King Edward, F.B.; Turriff, F B. 


\section{Plantaginacea.}

Plantago maritima, Linn.-Mortlach, B.G.

Littorella uniflora, Ashcers,-Alvah, B.G.

\section{ILLECEBRACEA.}

Scleranthus annuus, Linn.-Avah, B.G.; Turriff, F.B.; Grange, 8.A.; Mortlaoh, B.G.

\section{GHENOPODIACEE.}

Chonopodium album, Linn.-Turriff, F.B.

C. Bonus-Henricus, Linn.-Gamrie, F.B.; Alvah, B.G.; Turriff, F.B.; Inverkeithnie, B.G. : Mortlach, B.G.

Atriplex patula, Linn.-Turriff, F.B.

b. oreota (Huds.)-Gamrie, F.B.; King Edward, F.B.

Salsola Kali, Linn.-Oullen, 8.A.

\section{POLYGONACEA.}

Polygonum Hydropiper, Linn.-Gamrie, F.B.

P. Iapathifolium, Linn.-Gamrie, F.B.; Alvah, F.B.; King Edward, F.B.; Turriff, F.B.

P. amphibium, Linn.-Gamrie, F.B.; Alvah, F.B.; King Edward, F.B.; Grange, 8.A.; Mortlach, B.G.

P. Bistorta, Linn,-Boyndie, Ed.; Kirkmiohael, M.

P. viviparum, Linn.-Banff, S.A.; Alvah, B.G.; Turriff, \$.A.; Mortlach, B.G.

Oxyria digyna, Hill-Mortlach, B.G.

Rumex sanguineus, Linn., b. viridis, (Sibth.)-Gamrie, S.A.

R. Hydrolapathum, Huds.-Gamrie, 8.A.

ค. alpinus, Linn.-Turriff, F.B.

R. crispus, Linn. $x$ obtusifolius-Alvah, F.B.; King Edward, F.B.; Turriff, F.B.

R. domesticus, Hartm. $x$ obtusifolius-Gamrie, F.B.; Alvah, F.B.; King Edward, F.B.; Turriff, F.B.

\section{EUPHORBIACEE.}

Euphorbia Holioscopia, Linn.-Turrifr, F.B.

E. oxigua, Linn.-Boyndie, B.G.

Morcurialis perennis Linn.-Grange, B.A.

\section{URTIGAGEA.}

Humulus Lupulus, Linn.-Turriff, F.B.

Urtica diolica, Linn., b. angustifolia, Wimm. \& Grab.-Alvah, F.B.

u. urens, Linn.-Turriff, F.B.; Grange, B.A.

Cannabis sativa, Linn.-Banff, Ed.

\section{MYRICACEA.}

Myriea Gale, Linn.-Alvah, B.G.: Marnooh, B.G.

\section{CUPULIFERE.}

Botula alba, Linn.-Gamrie, F.B.; King Edward, F.B.

B. tomentesa, Reith \& $\Delta$ bel-Gamrie, F.B.

B. nana, Linn.-Kirkmiohael, B.G.

Alnus rotundifolia, Mill-Gamrie, F.B.

Corylus Avellana, Liun,-Gamrie, F.B.; King Edward, F.B.; Turriff, F.B.; Grange, 8.A. 
Quereus Robur, Linn.-Rathven, S.A.; Deskford, S.A.; Grange, S.A.

a. pedunculata, (Ehrh.)-Turriff, F.B.

Castanea sativa, Mill.-Alvah, F.B.; Turriff, F.B.

\section{SALICAGE}

salix pentandra, Linn.-Alvah, B.G.

S. fragilis, Linn.-King Edward, F.B.; Turriff, F.B.

S. alba, Linn,-King Edward, F.B.; Turriff, F.B.

s. purpurea, Linn.-Alvah, F.B.; King Edward, F.B.

S. viminalis, Linn.-Alvah, F.B.; King Edward, F.B.; Turriff, F.B.

s. caprea, Linn.-Gamrie, F.B.; Turriff, F.B. xphylicifolia, $8 m$.-Kirkmichael, $\mathbf{M}$.

8. aurita, Linn.-Gamrie, F.B.; Alvah, B.G.; King Edward, F.B.; Turriff, F. B $\times$ phylicifolia, Sm,-Kirkmiohael, $M$.

S. cinerea, Linn.-Turriff, F.B.

S. repens, Linn.-Turriff, F.B.

Populus tremula, Linn.-King Edward, F.B.(?)

P. nigra, Linn.-Turriff, F.B.

\section{EMPETRACEA.}

Empetrum nigrum, Linn.-King Edward, F.B.; Turriff, F.B.

\section{CONIFERF.}

Juniperus communis, Linn.-Oullen, B.G.; Alvah, B.G.

Larix europaea, DO.-Bellie, S.A.; Boyndie, S.A.

\section{HYDROCHARIDACEA.}

Elodea canadensis, Michx.-King Edward, F.B.

\section{ORCHIDACEAE.}

Listera cordata, Br.-Turriff, 8.A.; Mortlach, B.G.

L. ovata, Br.-Boharm, Ed.

Goodyera repens, Br.-Turriff, S.A.; Mortlach, B.G.

Helleborine atrorubens, Druce-Kirkmichael, M.

Orchis mascula, Linn.-Alvah, B.G.

0. Iatifolia, Linn.-Turriff, Skene; Kirkmichael, M.

o. maculata, Linn.-King Edward, F.B.

$\times$ latifolia-Kirkmiohael, M.

Habenaria conopsea, Benth.-Turriff, S.A.

H. albida, Br,-Alvah, B.G.

H. viridis, Br.-Alvah, B.G.; Mortlaoh, B.G.

\section{IRIDACEAE.}

Irls Psoudacorus, Linn,-Oullen, S.A.; King Edward, F.B.; Turriff, F.B.

\section{AMARYLLIDACEAE.}

Nareissus Pseudo-Naroissus, Linn.-Boyndie, Ed. 


\section{LILIACEA.}

Allium oloraceum, Linn,-Kirkmichnel, $\mathrm{M}$.

Scilla verna, Huds.-Rathven, \$.A.

S. non-scripta, Hoffmgg. \& Link.-Rathven, S.A.; Boyndie, Ed.; King Edward. S A.

Ornithogalum umbellatum, Linn.-Banfi, Ed.

Narthecium ossifragum, Ifuds.-King Edward, F.B.; Turriff, F.B.

Toneldia palustris, Huds.-Kirkmichael, M.

\section{JUNCACEAE.}

Juncus squarrosus, Linn.-King Edward, F.B.

J. maritimus, Linn.--Rathven, Ed.; Boyndie, Ed.

J. bulbosus, Linh.-Alvah, F.B.; King Edward, F.B.; Turriff, F.B.

d. articulatue, Linn.-King Edward, F.B.

J. sylvaticus, Reich.-Turriff, F.B.

J. biglumis, Linn.-Kirkmichael, B.G.

ง. triglumis, Linn.-Kirkmichael, M.

Luzula pilosa, Willd.-Gamrie, F.B.; King Edward, F.B.; Turriff, F.B.

L. spicata, DO.-Mortlach, B.G.

L. campestris, DO.-Gamrie, F.B.; Alvah, F.B.; King Edward, F.B.; Turriff F B: Grange, 8.A.

L. multifiora, DC., a. umbellata-King Edward, F.B.; Turriff, F.B.

b. congesta-Turriff, F.B.

TYPHACEE.

Typha latifolia, Linn.-Turriff, F.B.

Sparganium orectum, Linn,-Gamrie, F.B.

S. simplex, Huds.-Alvah, B.G.

s. affino, Sohnigl-Alvah, B.G.

5. minimum, Fr.-Turriff, F.B.

ARACER.

A rum maoulatum, Linn,-Rathven, Ed.

\section{LEMNACEF,}

Lemna minor, Linn.-Alvab, B.G.

L. trisulea, Linn.-Mortlach, B.G.

\section{ALISMACER.}

Alisma Plantago-aquatica, Linn.-Alvah, B.G.; King Edward, S.A.; Mortlaoh, B.G.

\section{NAIADACEA.}

Triglochin palustre, Iinn.-Turriff, F.B.

Potamogeton natans, Linn.-Gamrie, F.B.; Alvah, F.B.; Turriff, F.B.

P. polygonifolius, Pourr.-Turriff, F.B.

P. alpinus, Balb.-Alvah, B.G.; Turriff, S.A.; Kirkmichael, M.

P. Ianceolatus, Sm.-Alvah, B.G.; Mortlach, B.G.

P. Iucens, Linn.-Alvah, B.G.; Turrift, S.A.

P. perfoliatus, 8m.-King Edward, B.G.; Mortlach, B.G.

P. crispus, Linn.-Turriff, B.A.

P. pusillus, Iinn,-Alvah, B.G.

Ruppia rostellata, Kooh-Banfi, Ed., ex B.G. 


\section{CYPERACEA.}

Eleocharis palustris, Roem. \& Schult.-King Edward, F.B.

Scirpus cæespitosus, Isinn.-Gamrie, F.B.; King Edward, F.B.; Turriff, F.B.

s. sotaceus, Linn.-Alvah, B.G.; Mortlach, B.G.

s. Tabernaemontani, Gmel.-Bellie, Ed.; Rathven, Ed.

5. sylvatieus, Linn.-Alvah, B.G.

s. rufus, Sohrad.-Banff, B.G.

Eriophorum vaginatum, Linn,-King Edward, F.B.

E. angustifolium, Both.-King Edward, F.B.; Turriff, F.B.

Aynchospora alba, Vahl-King Edward, B.G.; Mortlach, B.G.

Schoenus nigricans, Linn.-Mortlach, B.G.

Carox dioica, Linn.-Alvah, B.G.

C. incurva, Lighte.-Banff, Bruce (v. Introd.)

C. pulicaris, Linn.-Alvah, B.G.; King Edward, F.B.; Turriff, F.B.

C. paucifiora, Lightf.-Kirkmichael, $\mathbf{M}$.

C. Boenninghausiana, Weihe-Bellie, B.G.

C. echinata, Murr.-King Edward, F.B.; Turriff, F.B.

c. curta, Good.-King Edward, F.B.

b. alpicola, Wahl.-Alvah, B.G.

C. Ioporina, Linn.-Turriff, F.B.

c. atrata, Linn.-Kirkmichael, M.

C. rigida, Good.-Mortlach, B.G.

C. aquatilis, Wahl.-Turriff, F.B.

C. Goodenowii, Gay-King Edward, F.B.; Turriff, F.B.

c. flacea, Sohreb.-King Edward, F.B.; Turriff, F.B.

C. rariflora, Smith-Kirkmichael, B.G.

C. caryophyllea, Latourr.-Turriff, 8.A.; Kirkmichael, M.

C. pallescens, Linn.-Kirkmichael, M.

C. panicea, Linn.-King Edward, F.B.; Turriff, F.B.

c. capillaris, Linn.-Kirkmichael, M.

C. pendula, Huds.-Gamrie, B.G.; Turriff, 8.A.

C. sylvatica, Huds.-Kirkmichael, M.

C. binervis, Sm.-Gamrie, F.B.; King Edward, F.B.

c. fulva, Host-Gamrie, F.B.; King Edward, F.B.; Kirkmichael, M.

C. oxtensa, Good.-Gamrie, B.G.

C. llava, Linn,-King Edward, F.B.; Turriff, F.B.

b. Iepidocarpa, (Tausch.)-Kirkmichael, $\mathbf{M}$.

$\times$ fulva-Kirkmichael, $M$.

C. Dederi, Retz., d. codocarpa, And.-Kirkmiohael, M.

C. xanthocarpa-Gamrie.

C. hirta, Linn.-Gamrie, S.A.

C. acutiformis, Ehrh.-Mortlach, B.G.

C. Inflata, Huds.-Gamrie, F.B.; King Edward, F.B.; Turriff, F.B.

\section{CRAMINEA.}

Phalaris arundinacea, Linn.-Kirkmichael, M.

Milium effusum, Linn.-Fordyce, Ed.

Phloum pratense, Linn., b. nodosum, (Linn.)-Gamrie, F.B.; Alvah, F.B.; King Edward,

P. arenarium, Linn,-Banff, N.F.

F.B.; Turriff, F.B.

Agrostis canina, Linn.-Gamrie, F.B.; King Edward, F.B.; Turriff, F.B. 
A. alba, Linn.-Turriff, F.B.

b. stolonifera, (Linn.)-Gamrie, F.B.; King Edward, F.B.; Turriff, F.B.

d. coarctata, Hoff m.-Kirkmichael, M.

A. pumila, Linn.-King Edward, F.B.: Turriff, F.B.: Oabraoh, B.G.

Deschampsia alpina, Roem. \& Sohult.-Kirkmichael, M.

Holous mollis, Linn,-Alvah, F.B.: Turriff, F.B.

Avena pubescens, Huds.-Mortlach, B.G.

A. pratensis, Linn.-Oullen, N.F.; Mortlach, B.G.

b. alpina, Smith-Kirkmiohael, B.G.

c. Iongifolia, Parn.-Kirkmichael, M.

A. strigosa, Schreb.-Gamrie, F.B.; King Edward, F.B.

A. Tatua, Linn.-Grange, S.A.

Sieglingia decumbens, Bernh,-King Edward, F.B.; Turriff, F.B.

Phragmites communis, Trin:-Alvah, B.G.

Kooleria gracilis, Pers.-Alvah, B.G.; Grange, S.A.; Mortlach, B.G.

c. britannica, Domin-Kirkmichael, M.

Molinia coerulea, Moenoh.-Alvah, B.G.; Turriff, F.B.

Melica nutans, Linn.-Kirkmiohael, M.; Keith, B.G.

Briza modia, Linn.-Alvah, B.G.

Poa alpina, Linn.-Kirkmiohael, M.

P. nemoralis, Linn.-Turriff, F.B.

d. divaricata, syme-Kirkmichael, $\mathbf{M}$.

P. pratensis, Linn.-King Edward, F.B.

P. trivialis, Linn.-Turriff, F.B.

Clycoria aquatica, Wablb,-Alvah, F.B.; King Edward, F.B.; Turrif, F.B.

C. declinata, Bréb.-Kirkmiohael, M.

Festuca bromoides, Linn.-Turrifr, F.B.

F. ovina, Linn,-King Edward, F.B.

F. rubra, Linn.-Alvah, F.B.; King Edward, F.B.

F. sylvatica, Vill.-Keith, N.F.

F. pratensis, Huds.-Alvah, F.B.

F. elatior, Linn.-Gamrie, F.B.; Alvah, B.G.; Turriff, F.B.; Mortlach, B.G.

Bromus giganteus, Linn.-Boyndie, B.G.; Gamrie, F.B.; Alvah, F.B.; Keith, N.F.

B. ramosus, Huds.-Qamrio, F.B.; Alvah, S.A.

B. Sterilis, Linn.-Banff, N.F.; Alvah, B.G.; Mortlach, B.G.

B. Secalinus, Linn.-Mortlach, Ed.

B. commutatus, Schrad.-Gamarie, F.B.; Alvah, F.B.; King Edward, F.B.; Turriff, F.B.

B. hordoacous, Linn.-King Edward, F.B.; Turriff, F.B.

Brachypodium sylvaticum, Roem. \& Schult.-Alvah, B.G.; King Edward, F.B.; Koith, N.F.

Agropyron caninum, Beauv.-Turriff, F.B.; Kirkmichael, M.

A. repens, Beauv., b. Leersianum, Gray-Gamrie, F.B.; King Edward, F.B.; Turriff, F.B.

Nardus striota, Linn.-King Edward, F.B.; TurrifP, F.B.

FILICES.

Asplenium Adiantum-nigrum, Linn.-Banff, Ed.; Alvah, B.G.; Mortlaoh, B.G.

A. Triohomanes, Iinn.-Gamrie, F.B.; Mortlach, B.G.

A. Ruta-muraria, Linn.-Banff.

Athyrium Filix-fomina, Roth-King Edward, F.B.

Phyllitis Scolopendrium, Newm.-Gamrie, S.A.; Turriff, B.G.

Cystopteris fragilis, Bernh.-Gamrie, B.G.; Alvah, B.G.; Turrif, S.A.; Mortlach, B.G.

b. dentata, Hook.-Kirkmichael, M.; Mortlach, B,G.

Polystichum lobatum, Presl.-Gamrie, F.B.; Turriff, B.G. 
Lastrea spinuiosa, Presl.-Kirkmiohael, M.

Polypodium vulgare, Linn--Turriff, F.B.

Phegopteris Dryopteris, Fée-Gamrie, F.B.; Turriff, S.A.

P. polypodioides, Fée-Gamrie, F.B.; Alvah, B.G.; King Edward, B.G.

Ophioglossum vulgatum, Linn.-Gamrie (Flora of Buchan, by Mr R. Walker).

Botrychium Lunaria, Sw.-Fordyce, Ed.; Mortlach, B.G.

\section{EQUISETACEA.}

Equisetum sylvaticum, Linn.-Turriff, F.B.

E. palustre, Linn.-Aivah, F.B.; Turriff, F.B.

E. limosum, Linn,-Turriff, F.B.

\section{LYCOPODIACEF.}

Lycopodium annotinum, Linn.-Fordyce, Ed.; Kirkmichael, M.; Aberlour, B.G.

L. clavatum, Linn.-Gamrie, F.B.

L. alpinum, Linn.-Alvah, B.G.

b. decipiens, Syme-Kirkmiohael, M.

\section{CHARAGEA.}

Nitella opaca, Agardh-Kirkmichael, M. 


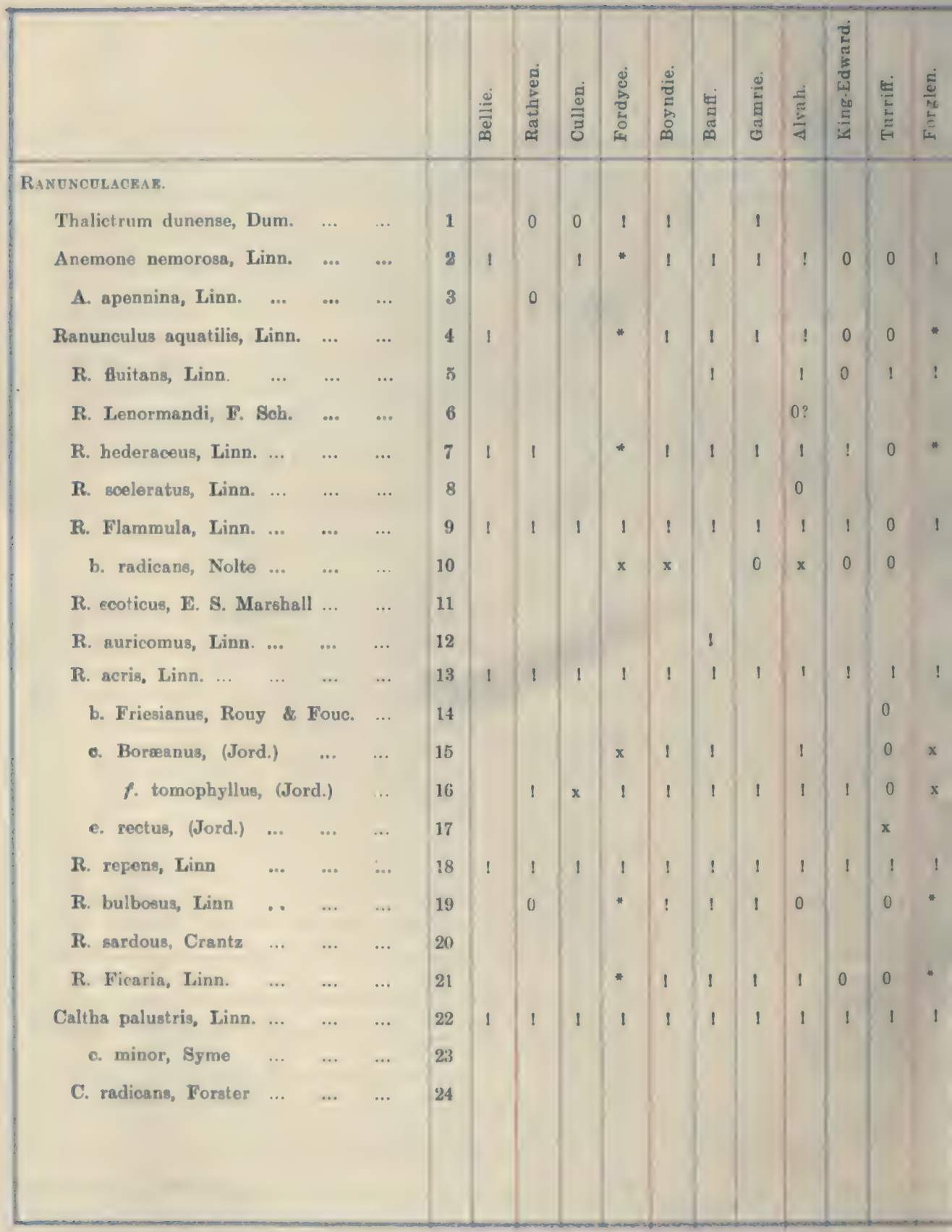




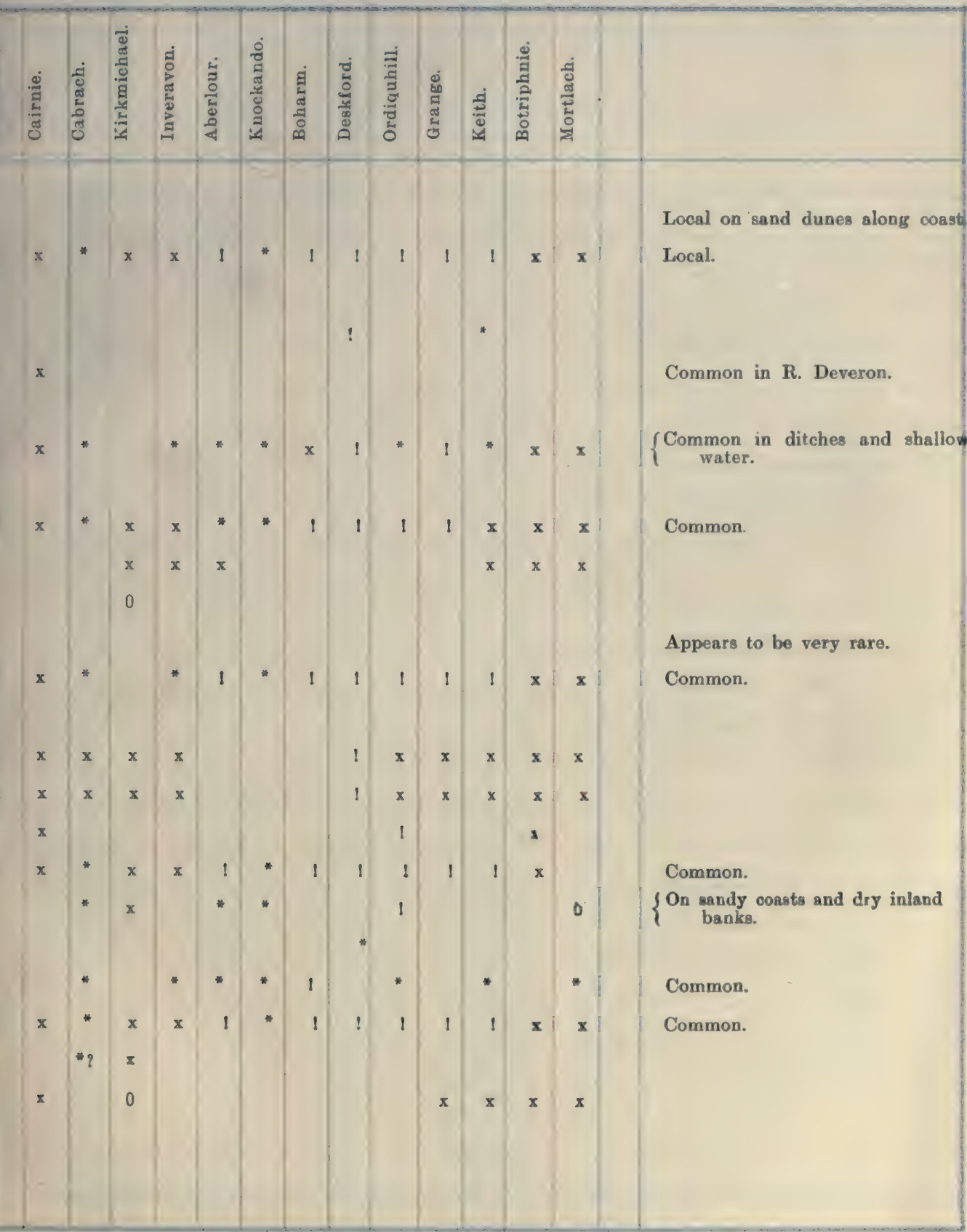




\section{RanUNCULACrar-Contá.}

Trollius europreus, Linn.

Eranthis hyemalis, Linn.

Aquilegia vulgars, Linn.

Aconitum Napellus. Linn.

\section{Berrgridacear.}

Berberis vulgaris, Lunn.

NYMPIARACEAE.

Nymphæa lutea, Linn.

Castalia alba, Wood

\section{Papaveraceae}

\begin{tabular}{llll|l} 
Papaver somniferum, Linn. & $\ldots$ & $\ldots$ & 8
\end{tabular}

P. Rhœeas, Linn.

P. dubium, Linn

Meconopsis cambrica, Vig.

Chelidonium majus Linn. FUMARIACEAR.

Corydalie claviculata, DC. ... . 19

Fumaria capreolata, Linn. $\quad \ldots \quad$... $\quad 14$

\begin{tabular}{lllll|l} 
F. Bastardi, Bor. & $\ldots$ & $\ldots$ & $\ldots$ & 15
\end{tabular}

$\begin{array}{llllll}\text { F. Officinalis, Linn. } & . . & \ldots & . . & 16\end{array}$ Crucifarar.

$\begin{array}{lllll}\text { Cheiranthus Cheiri, Linn. } & \ldots & \ldots & 17\end{array}$

Nasturtium officinale, R. Br. 


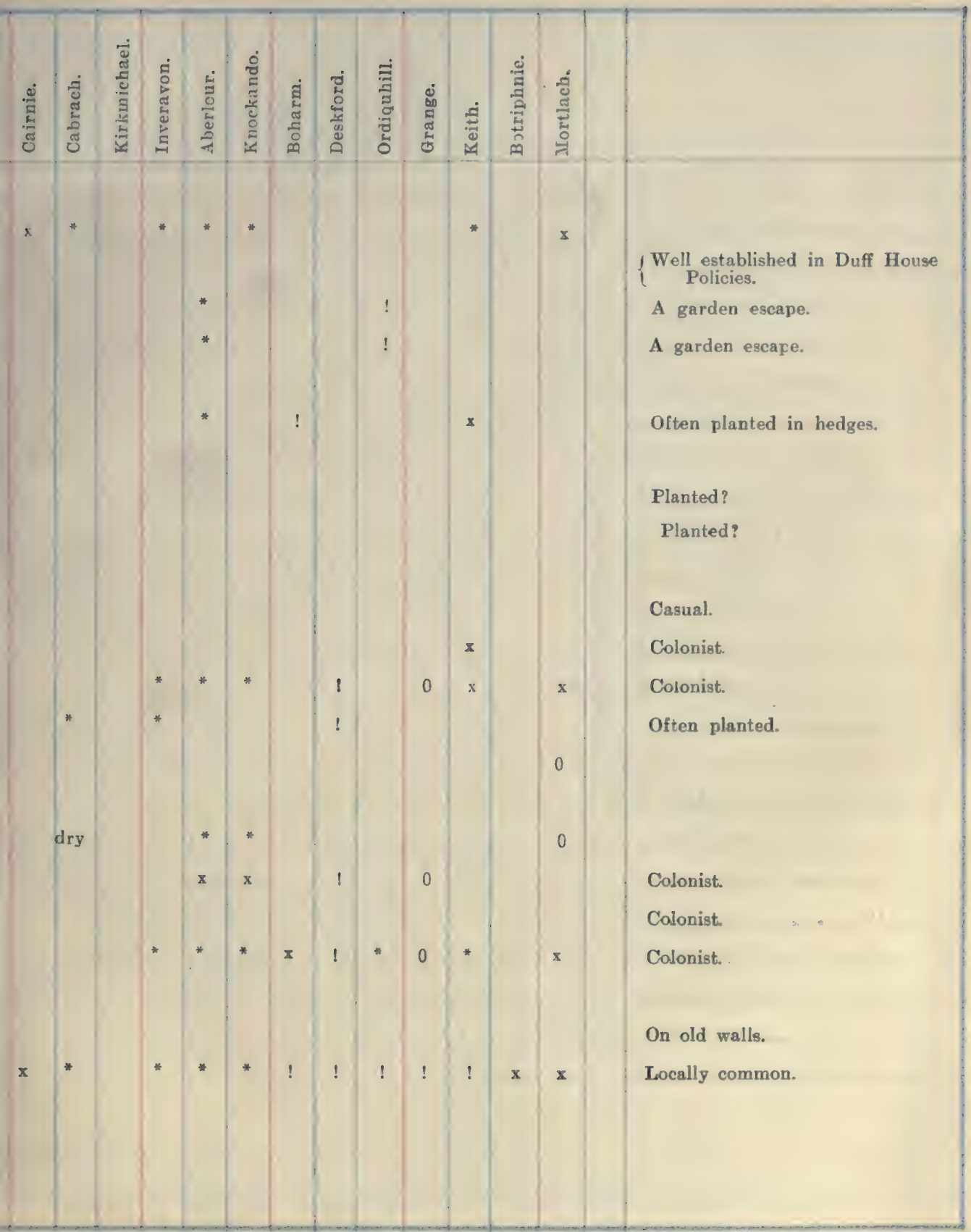




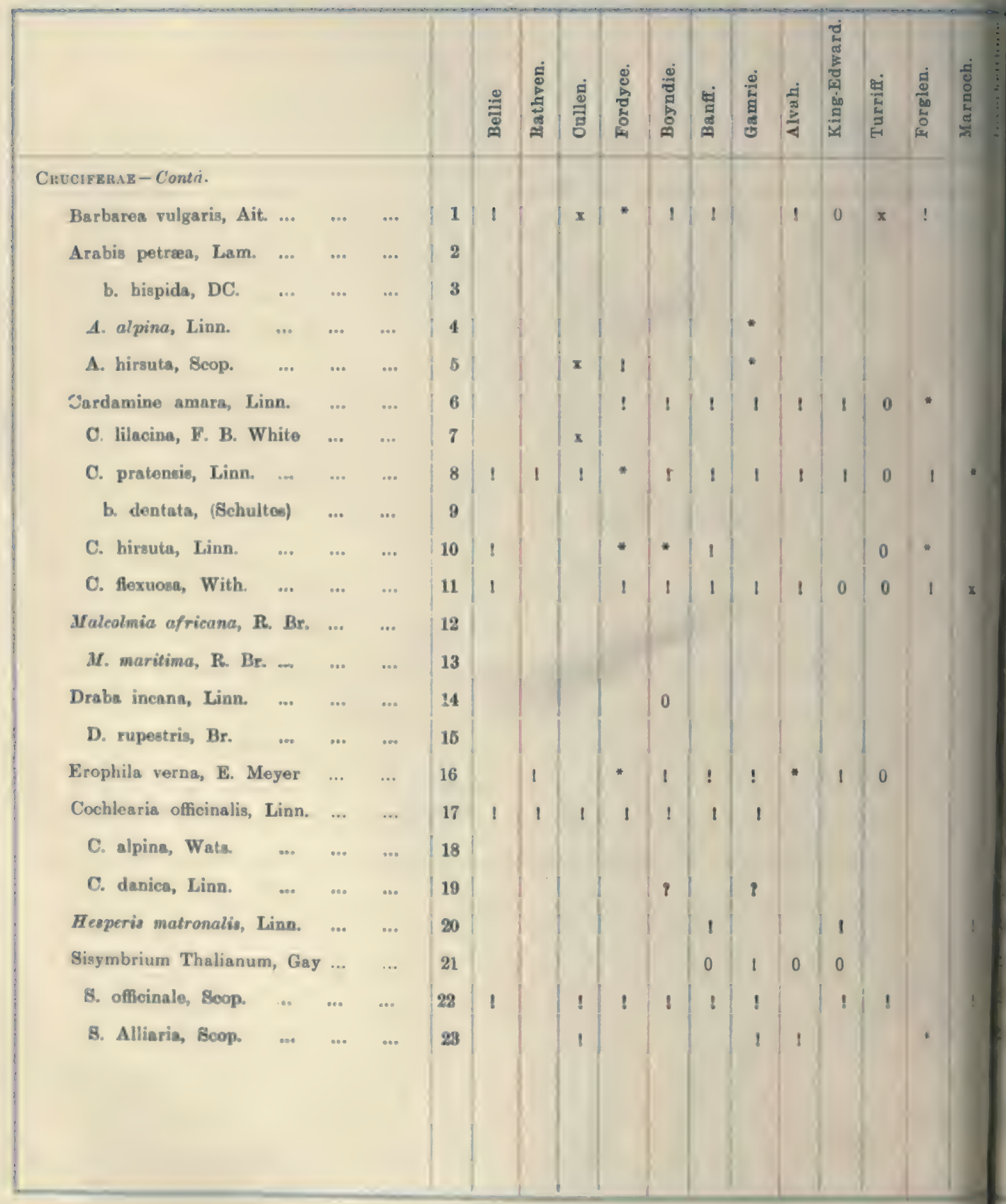




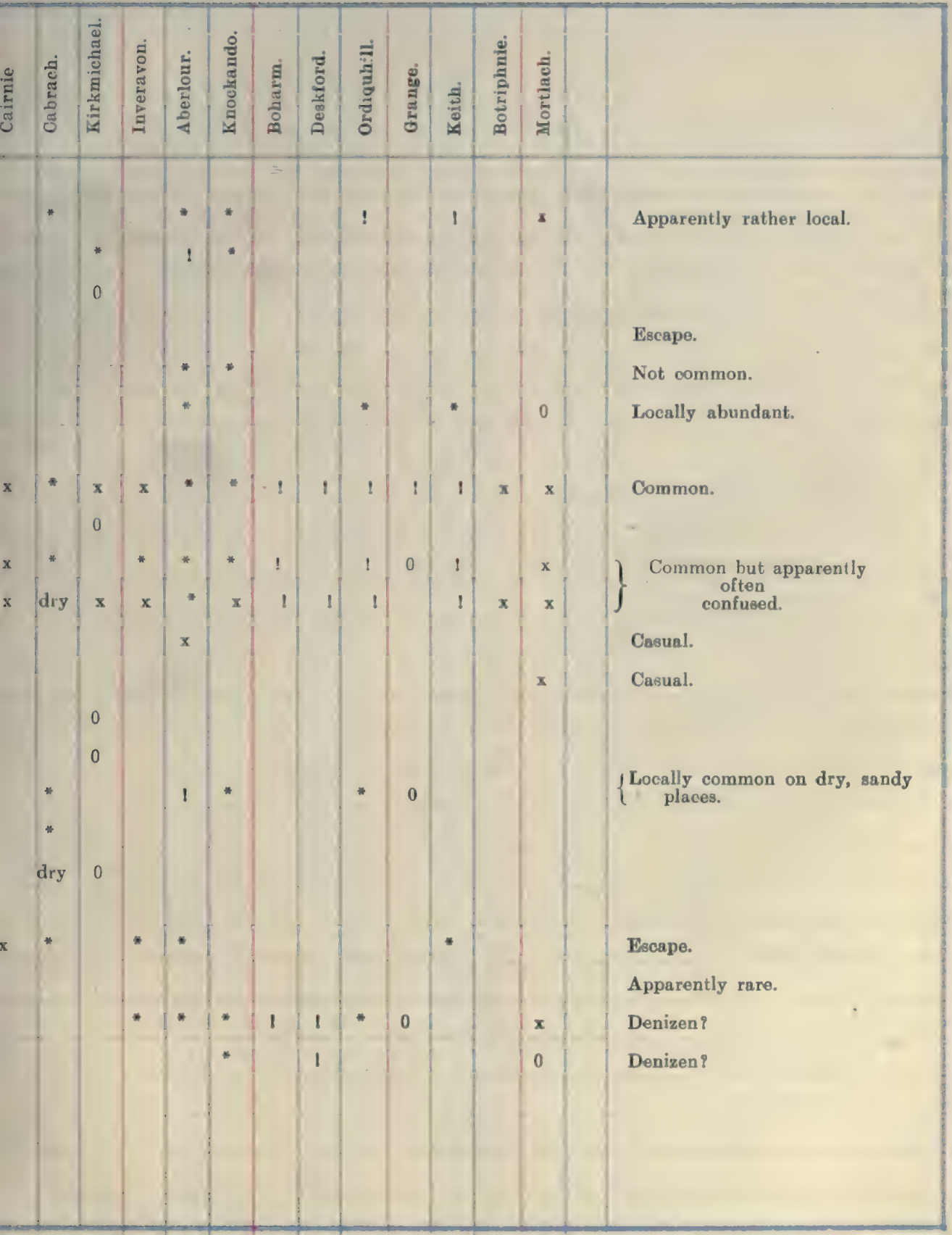




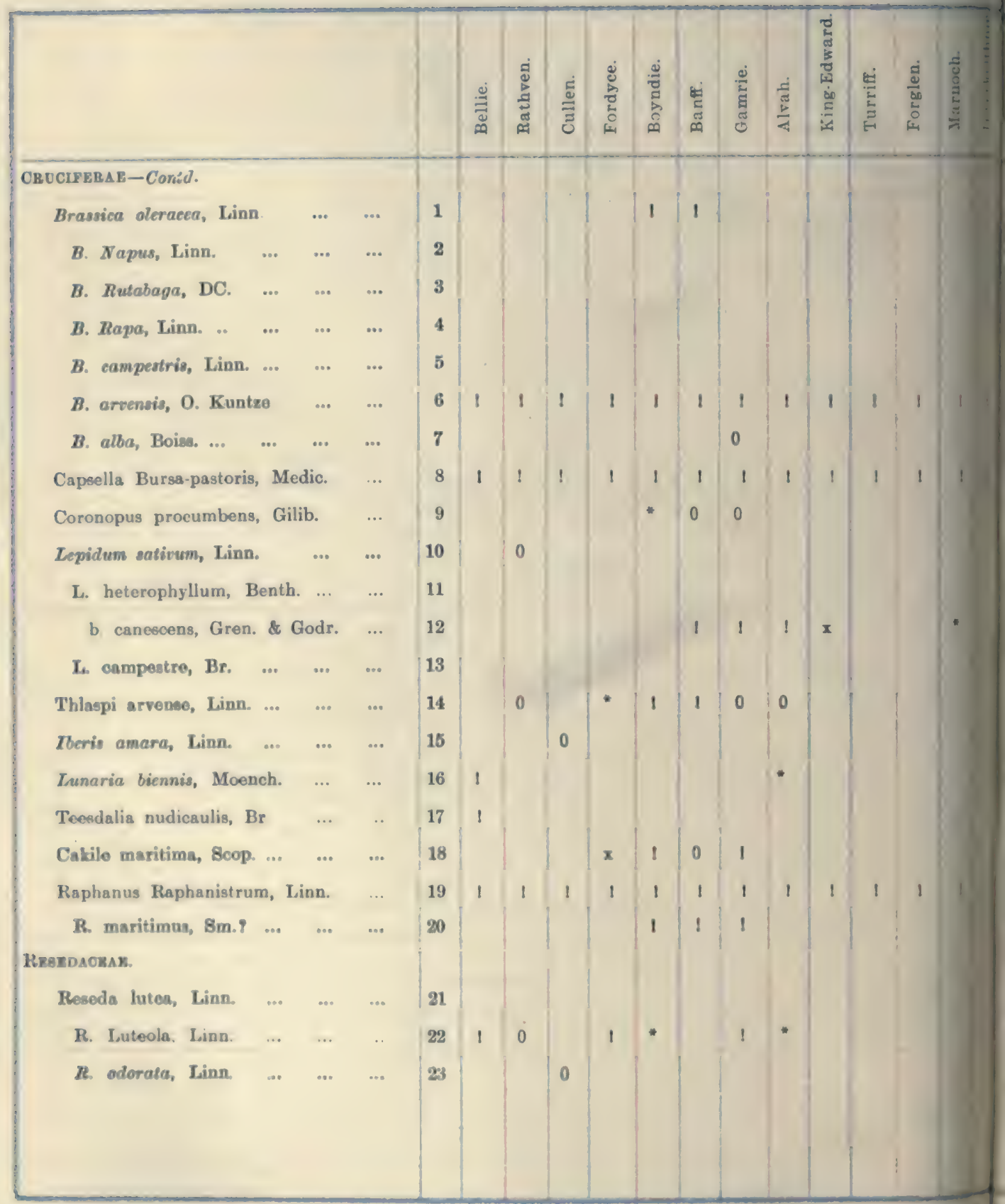




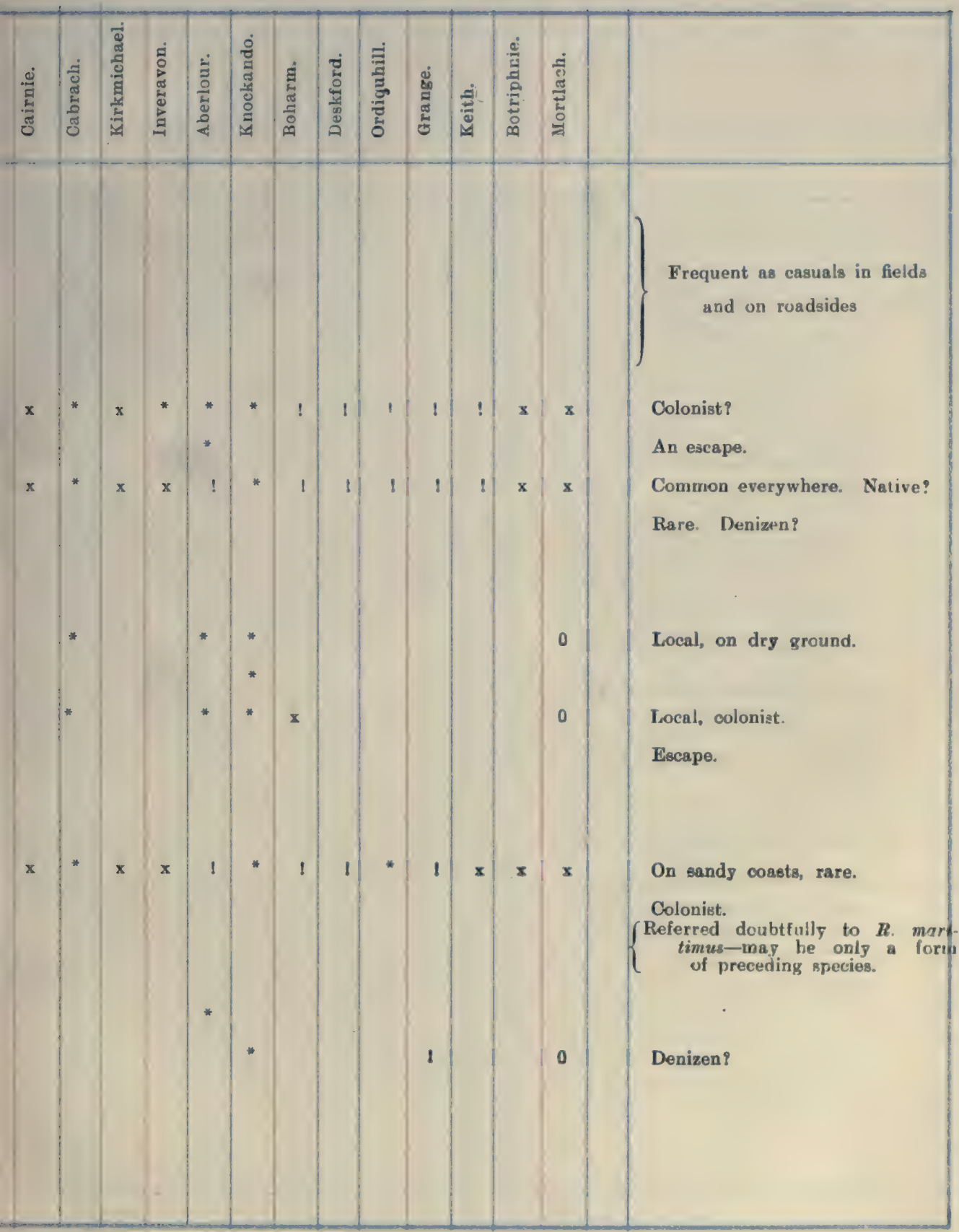




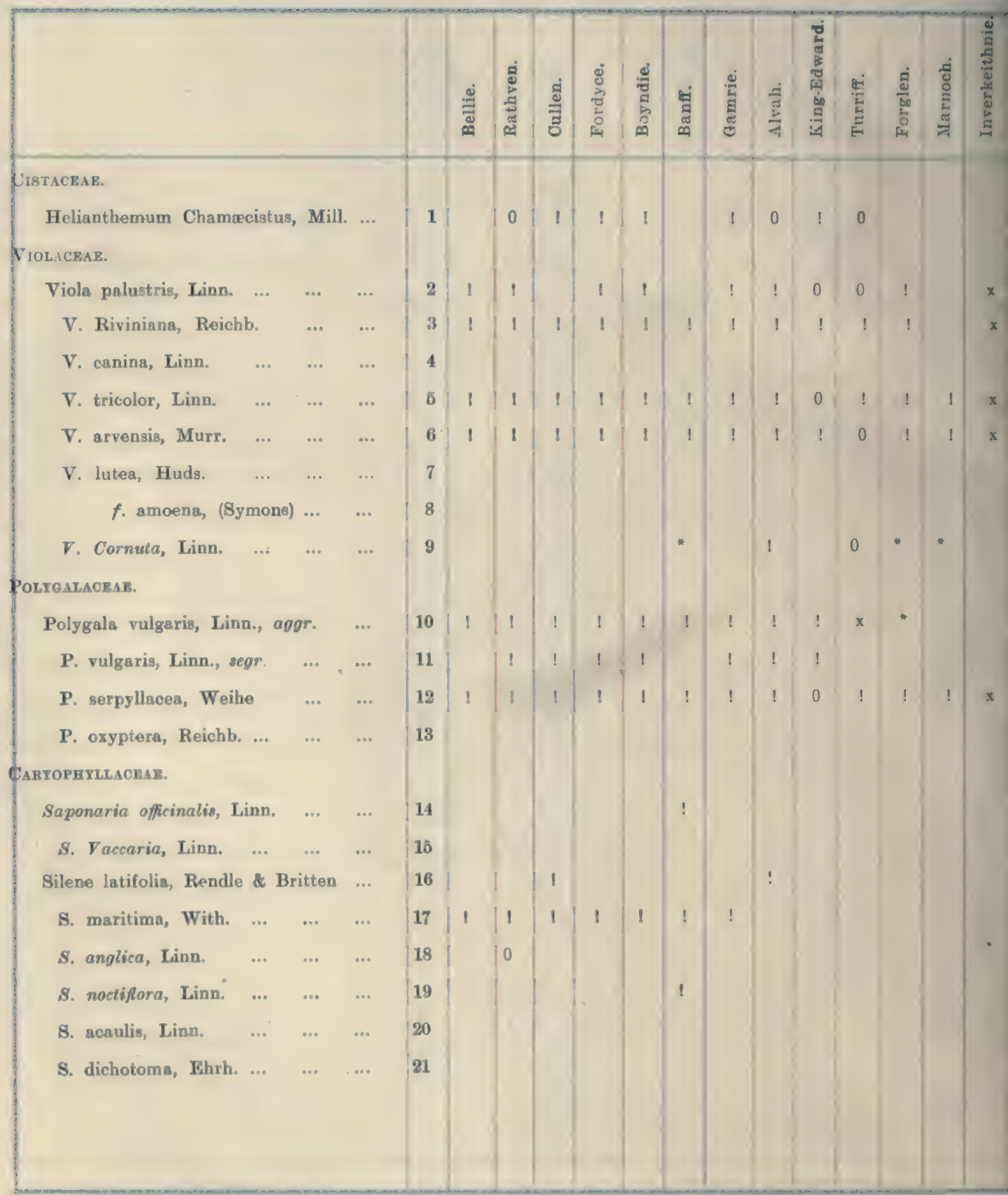




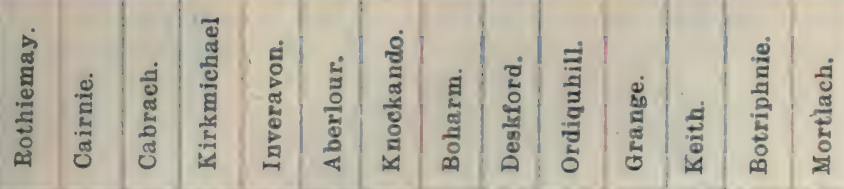

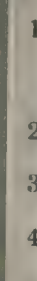

1

\begin{tabular}{l|l|l|l|l|l|l|l|}
1 & & $*$ & $d r y$ & $x$ & $*$ & \\
2 & 1 & $x$ & $*$ & $x$ & $x$ & $*$ & $*$ \\
3 & $!$ & $x$ & $d r y$ & $x$ & $x$ & 1 & $*$ \\
4 & & & & $x$ & & $*$ & $x$
\end{tabular}

\begin{tabular}{l|l|l|l|l|l|l|l|}
1 & & $*$ & $d r y$ & $x$ & $*$ & \\
2 & $!$ & $x$ & $*$ & $x$ & $x$ & $*$ & $*$ \\
3 & $!$ & $x$ & $d r y$ & $x$ & $x$ & $!$ & $*$ \\
4 & & & & $x$ & & $*$ & $x$
\end{tabular}

7

8

10
11
12
13
14
15

16

17

18

19

20

21

(1)

! $\mathbf{x}$

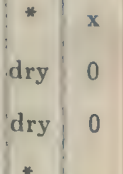

.
101

x

0

\begin{tabular}{l|l|l|l|l|l|l|}
1 & 1 & 1 & 1 & 1 & $x$ & $x$
\end{tabular}

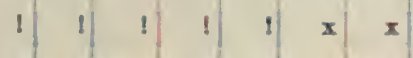

\begin{tabular}{|l|l|l|l|l|l|} 
& & & & & \\
1 & 1 & 1 & 1 & $\mathbf{x}$ & $\mathbf{x}$
\end{tabular}

\begin{tabular}{|l|l|l|l|l|l|l|l|l|l|l|l}
$*$ & $\mathbf{x}$ & $\mathbf{x}$ & 1 & $*$ & 1 & 1 & 1 & 1 & 1 & $\mathbf{x}$ & $\mathbf{x}$ \\
\hline
\end{tabular}

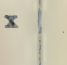

$\mathbf{x}$
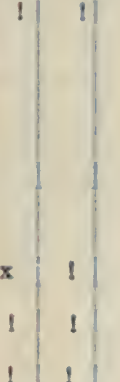


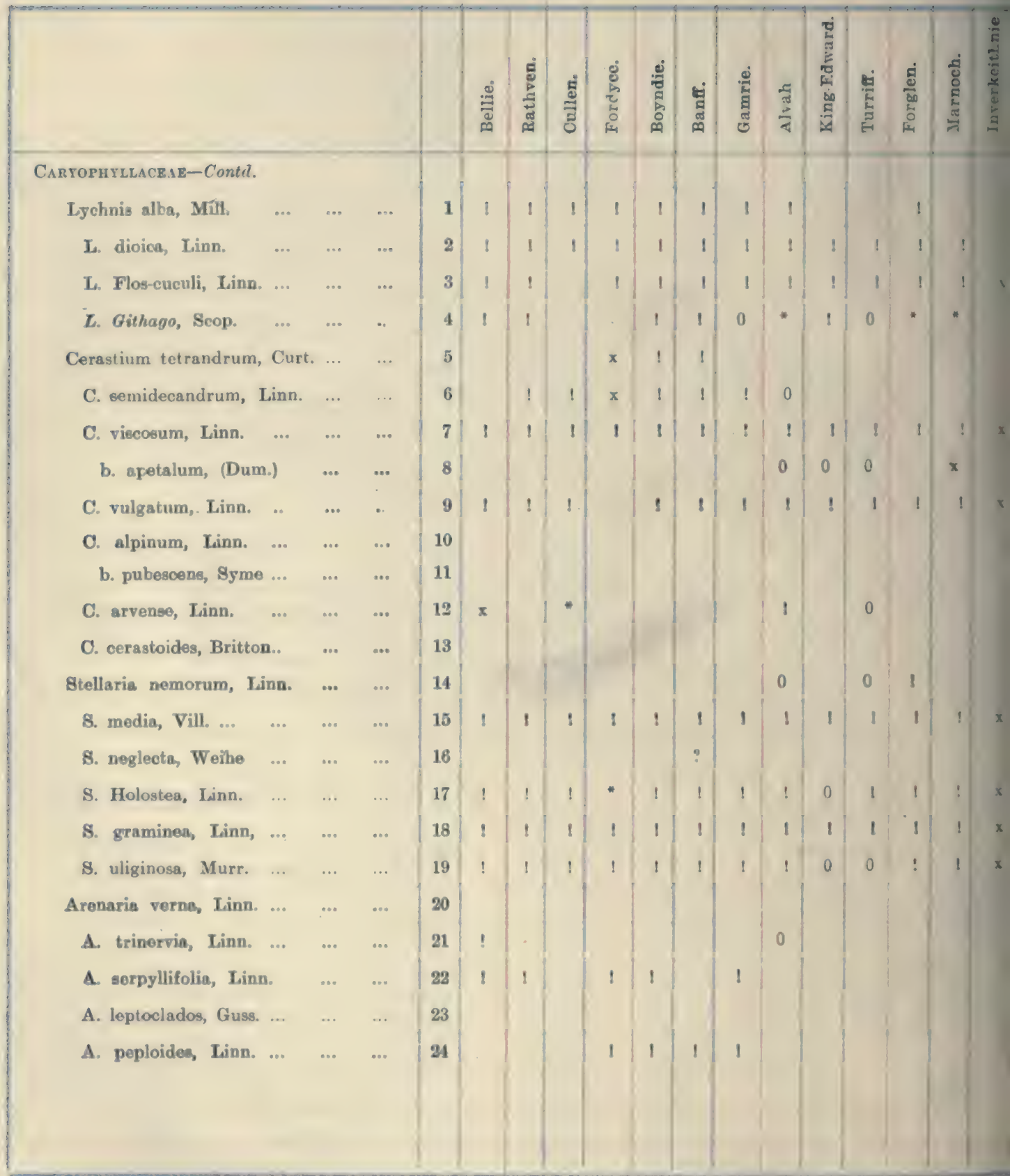




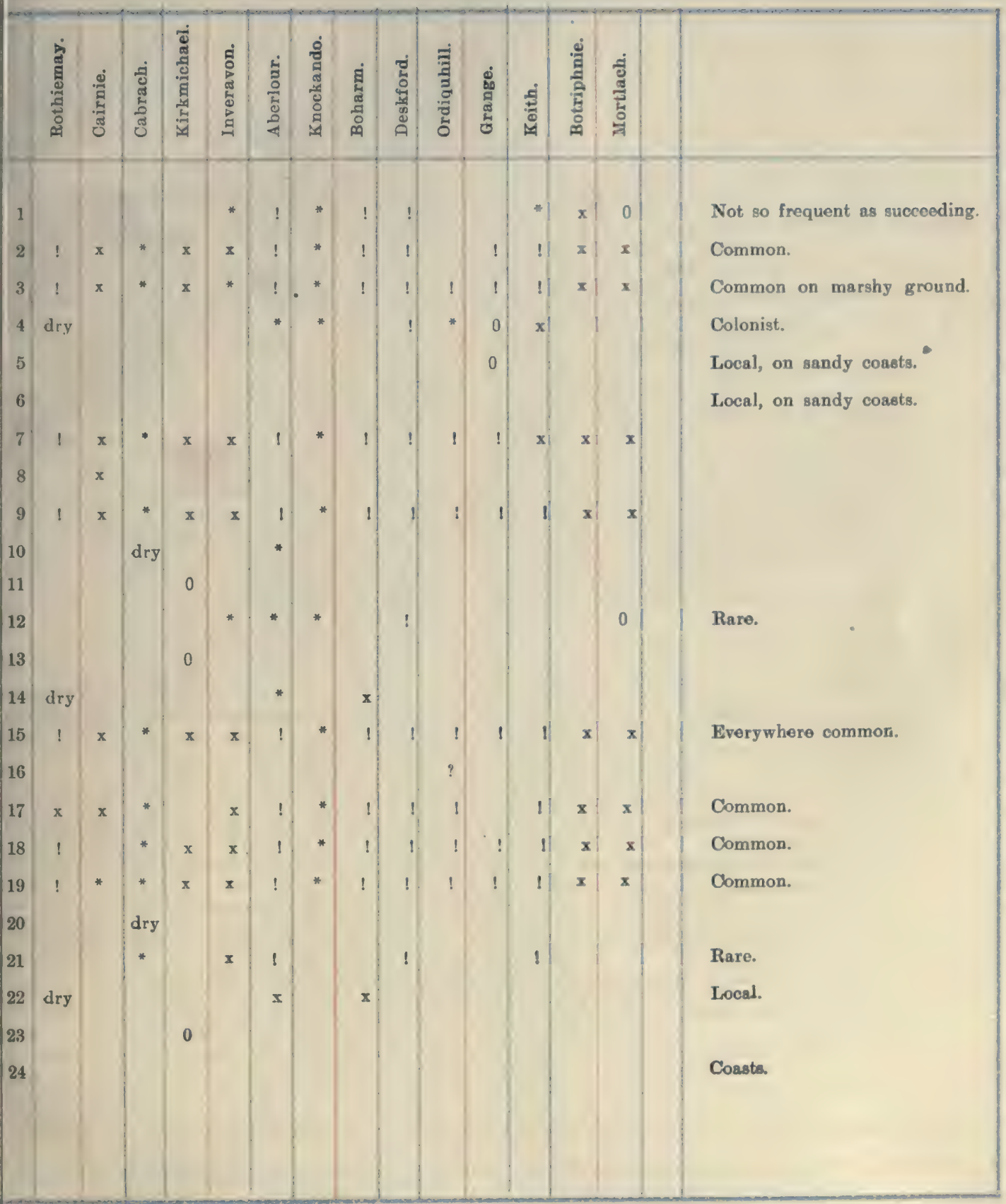




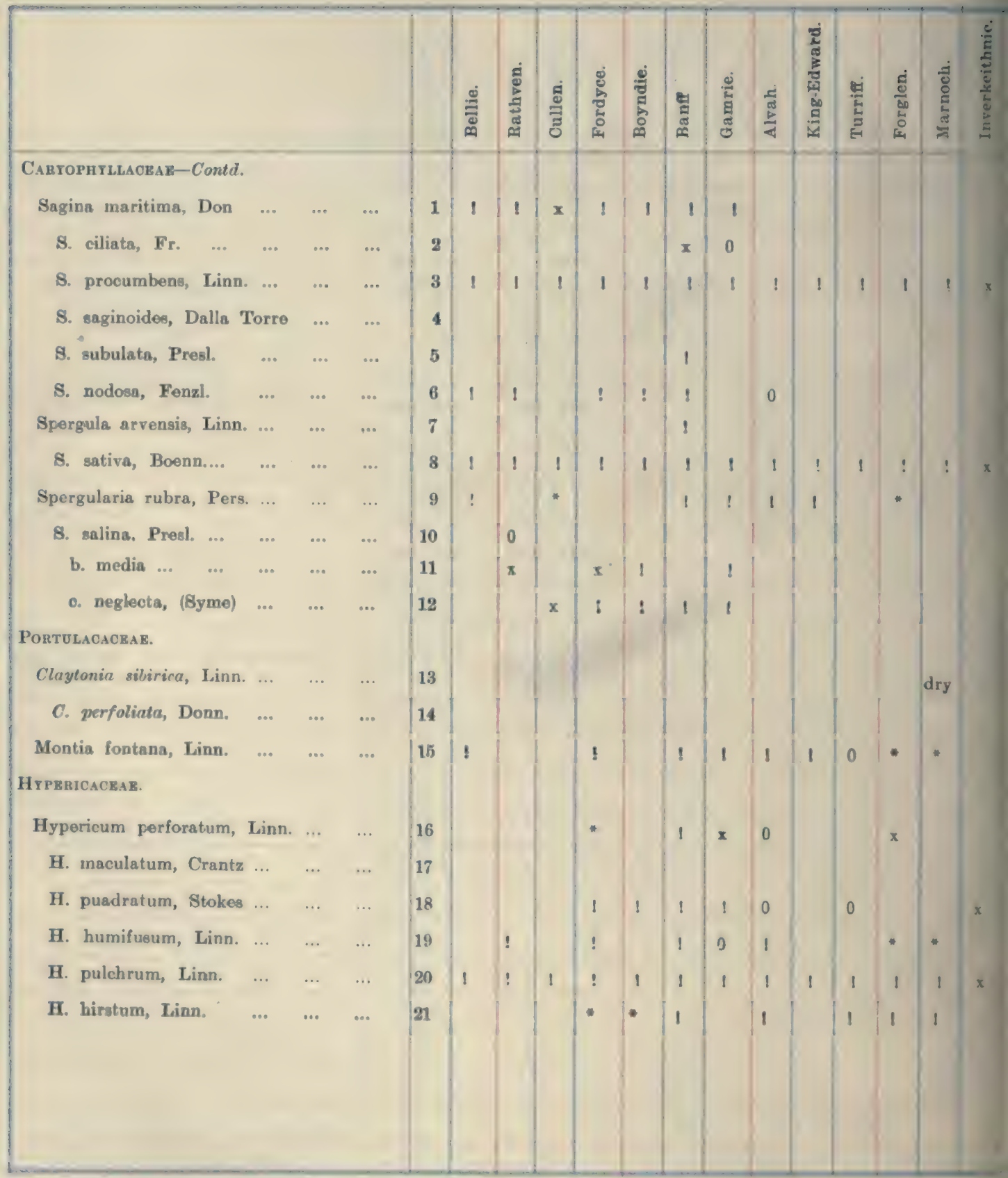




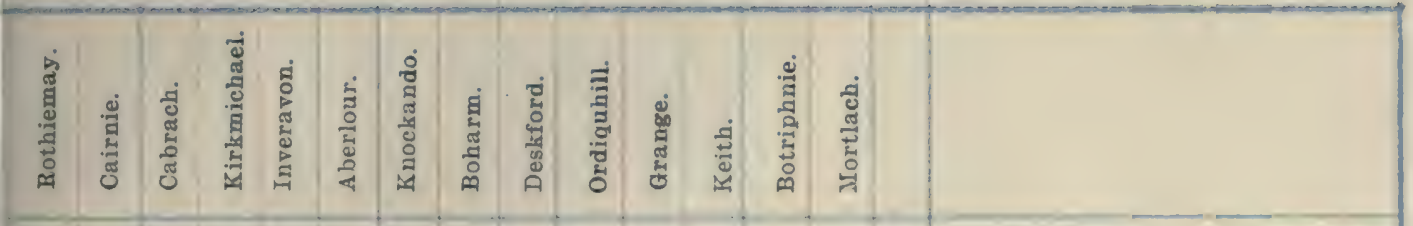

Coasts.

Coasts, rare.

Everywhere common.

Rare.

Local on wet links and moors.

Rare, Colonist.

Common, Colonist.

Alien.

Aleb.

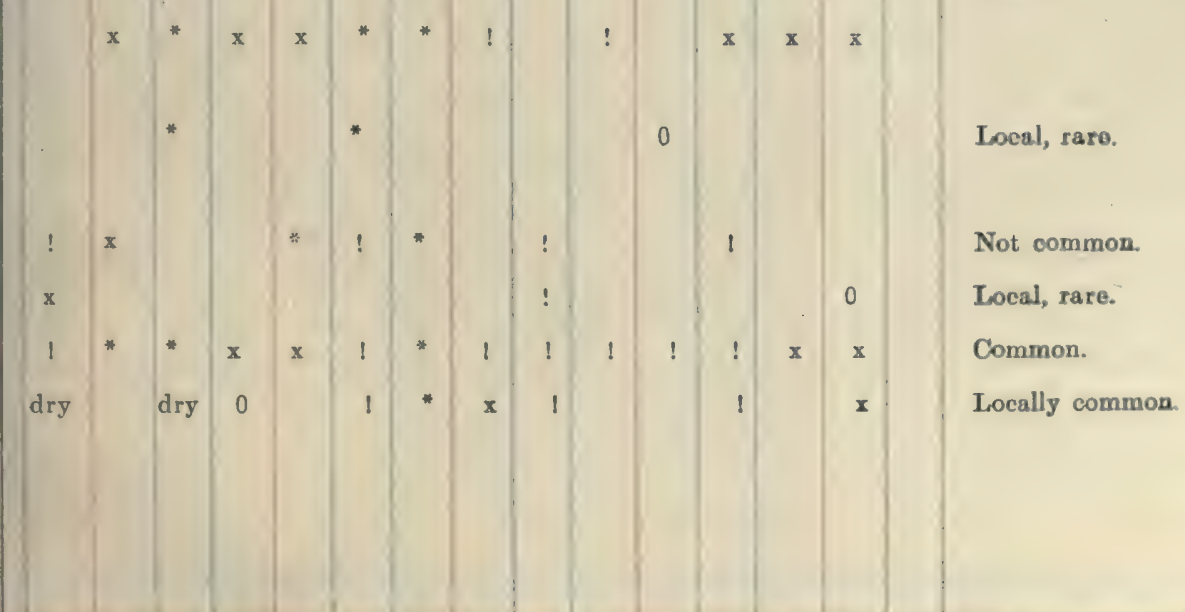




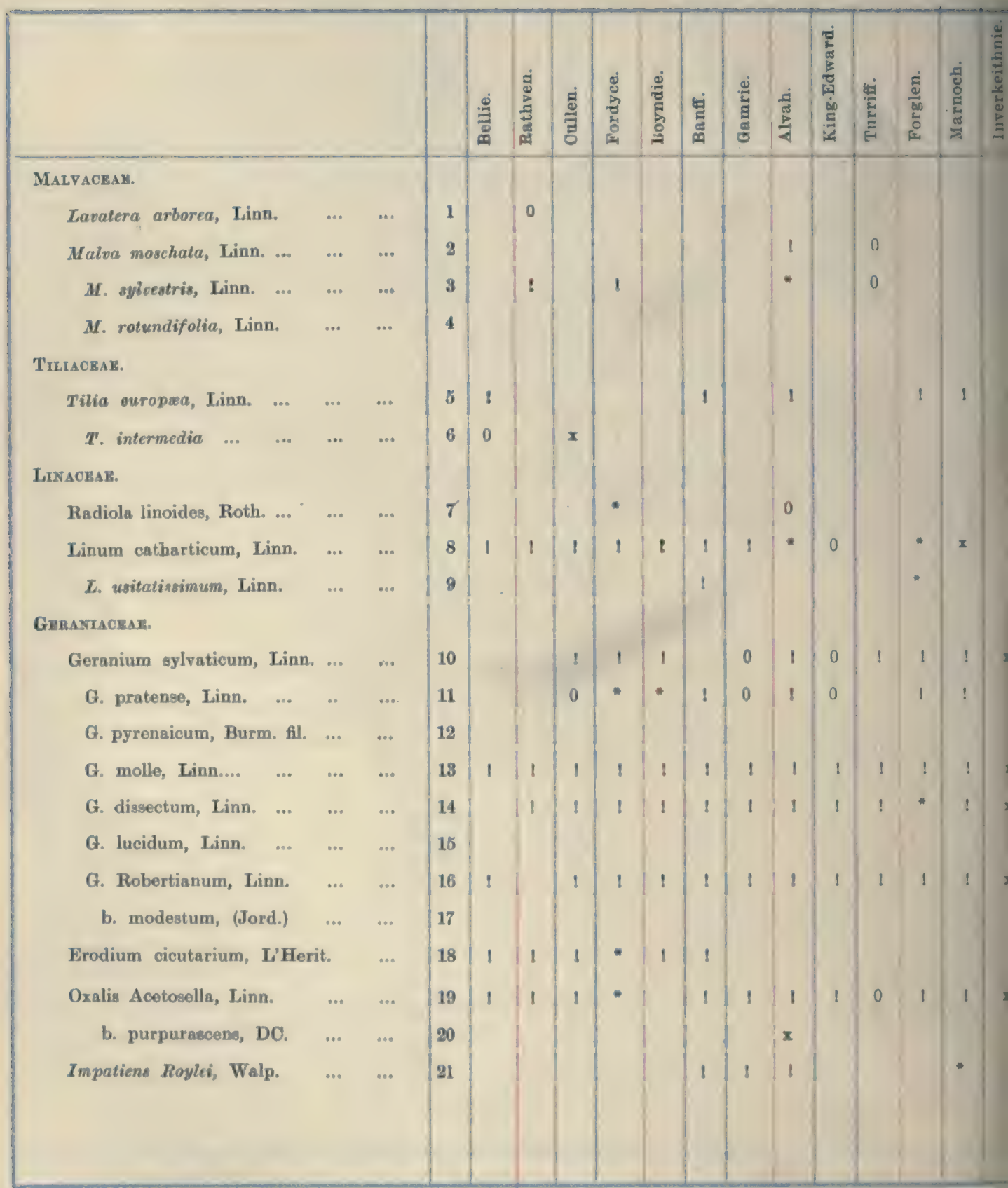




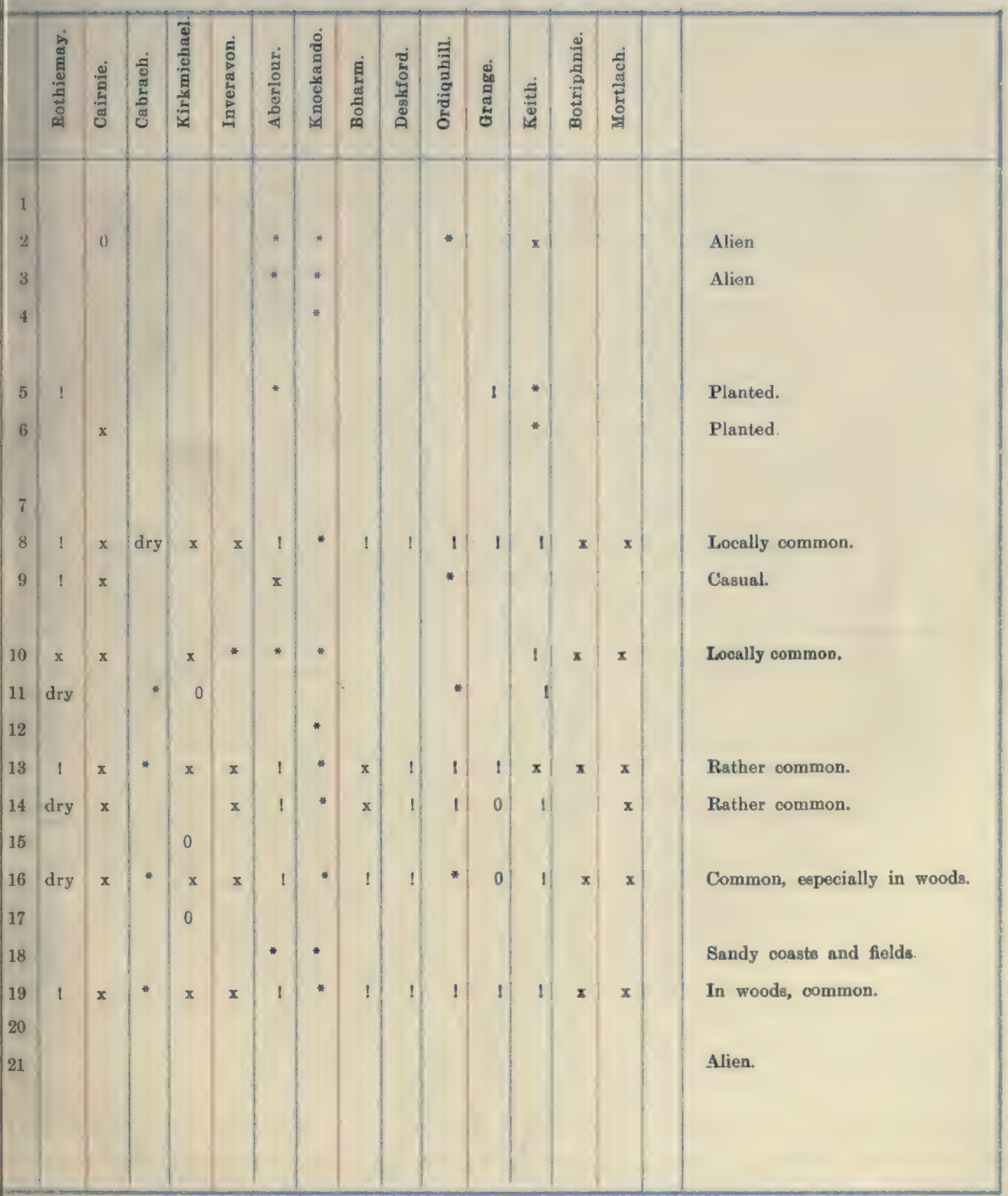




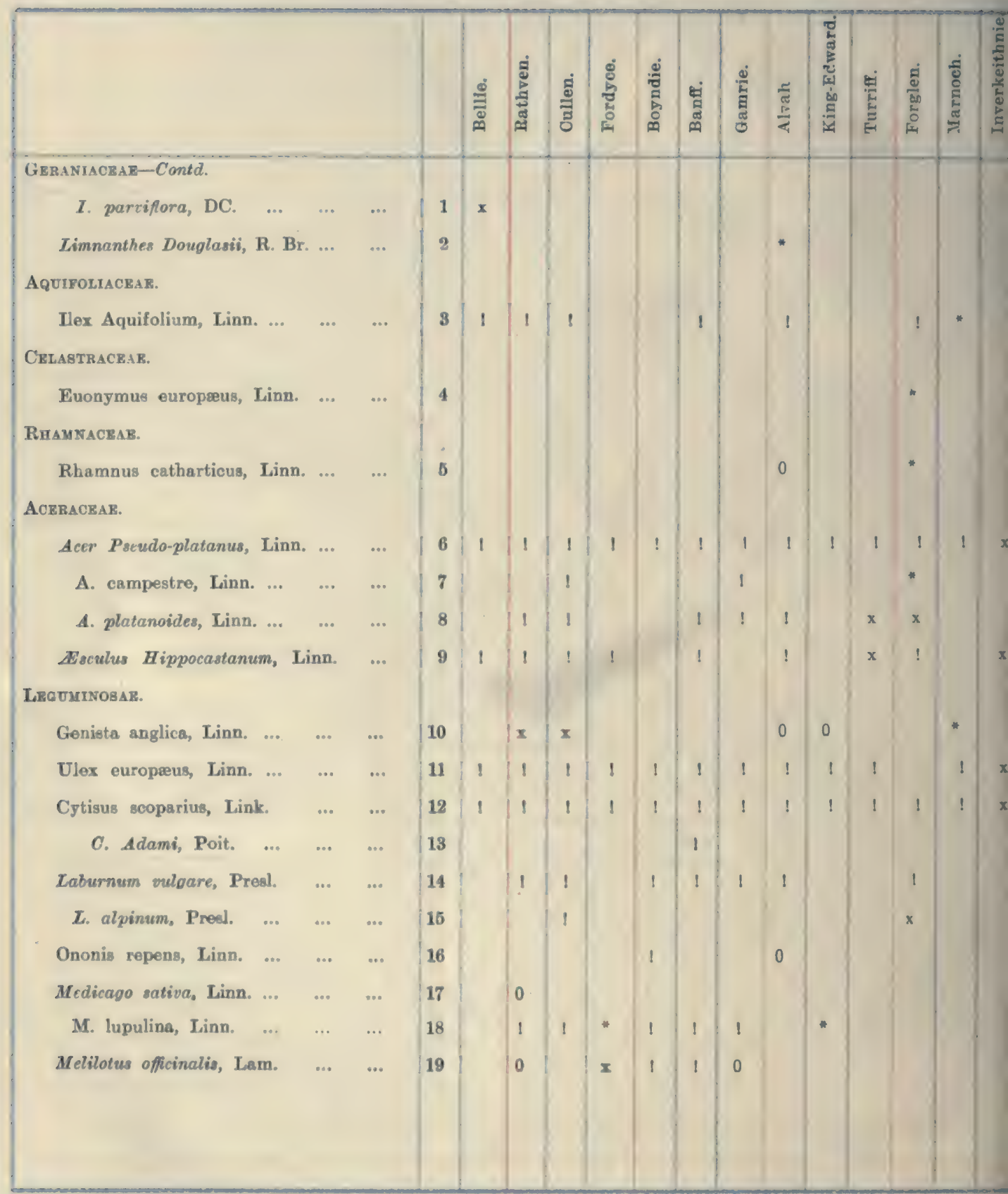




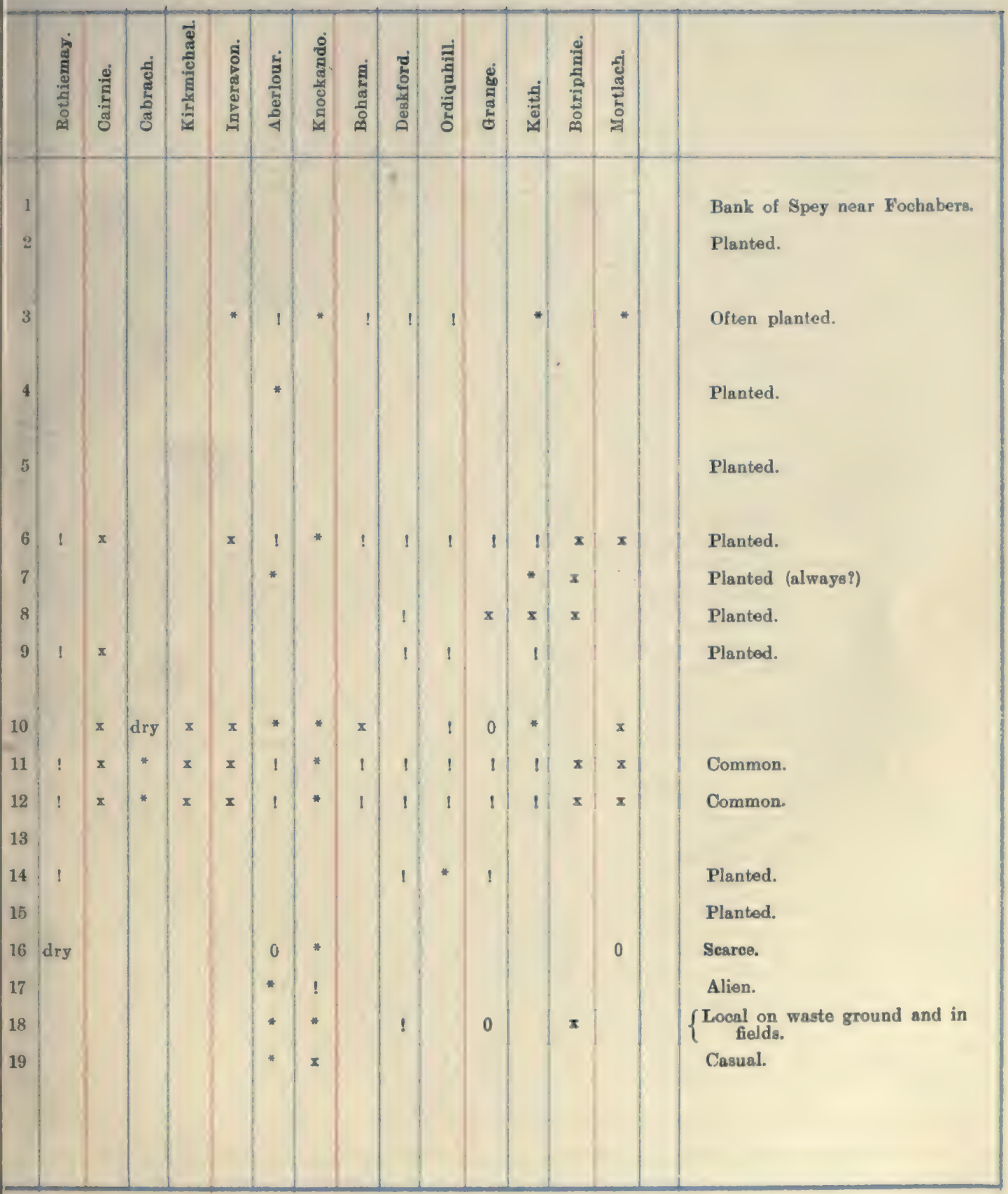




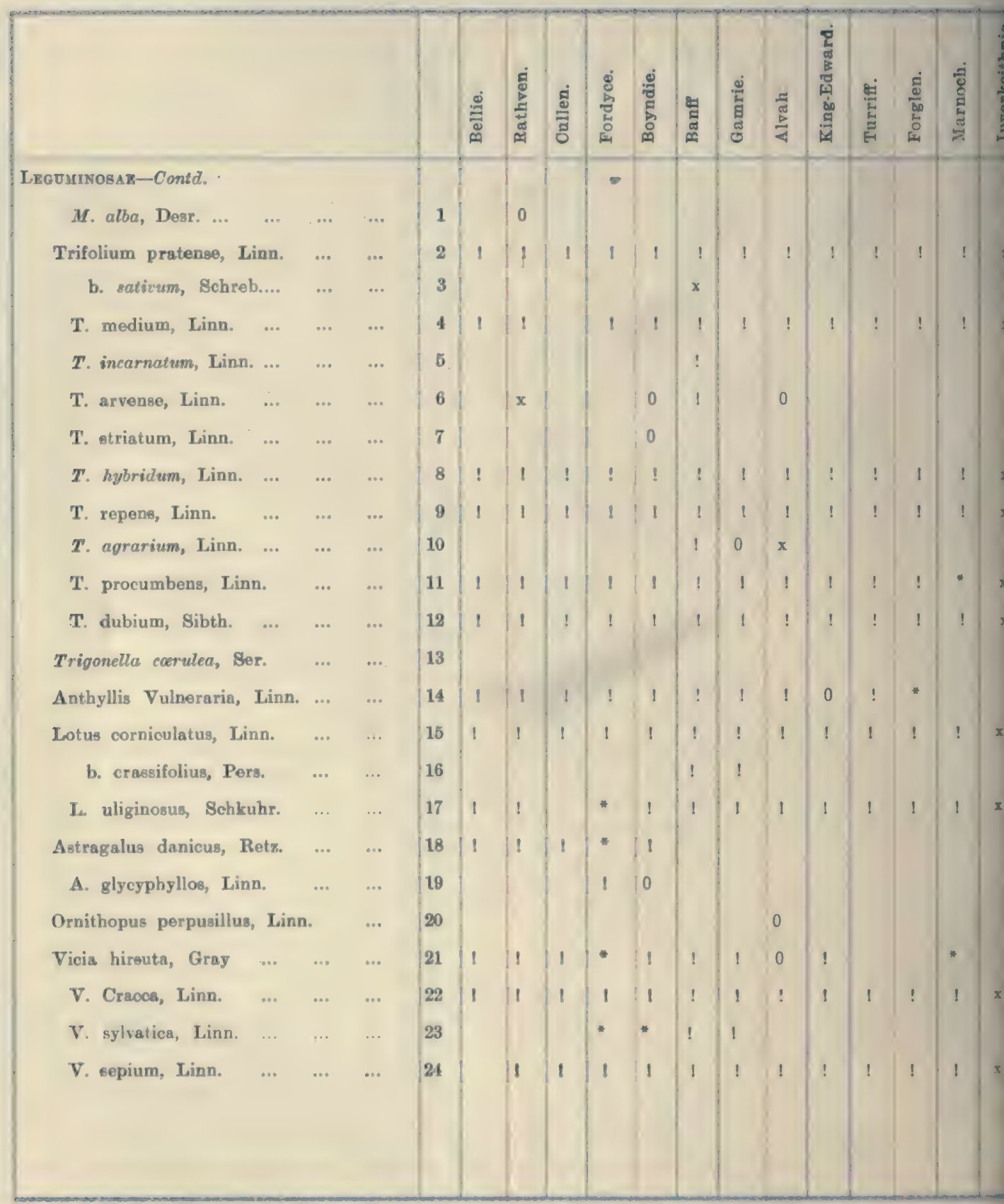




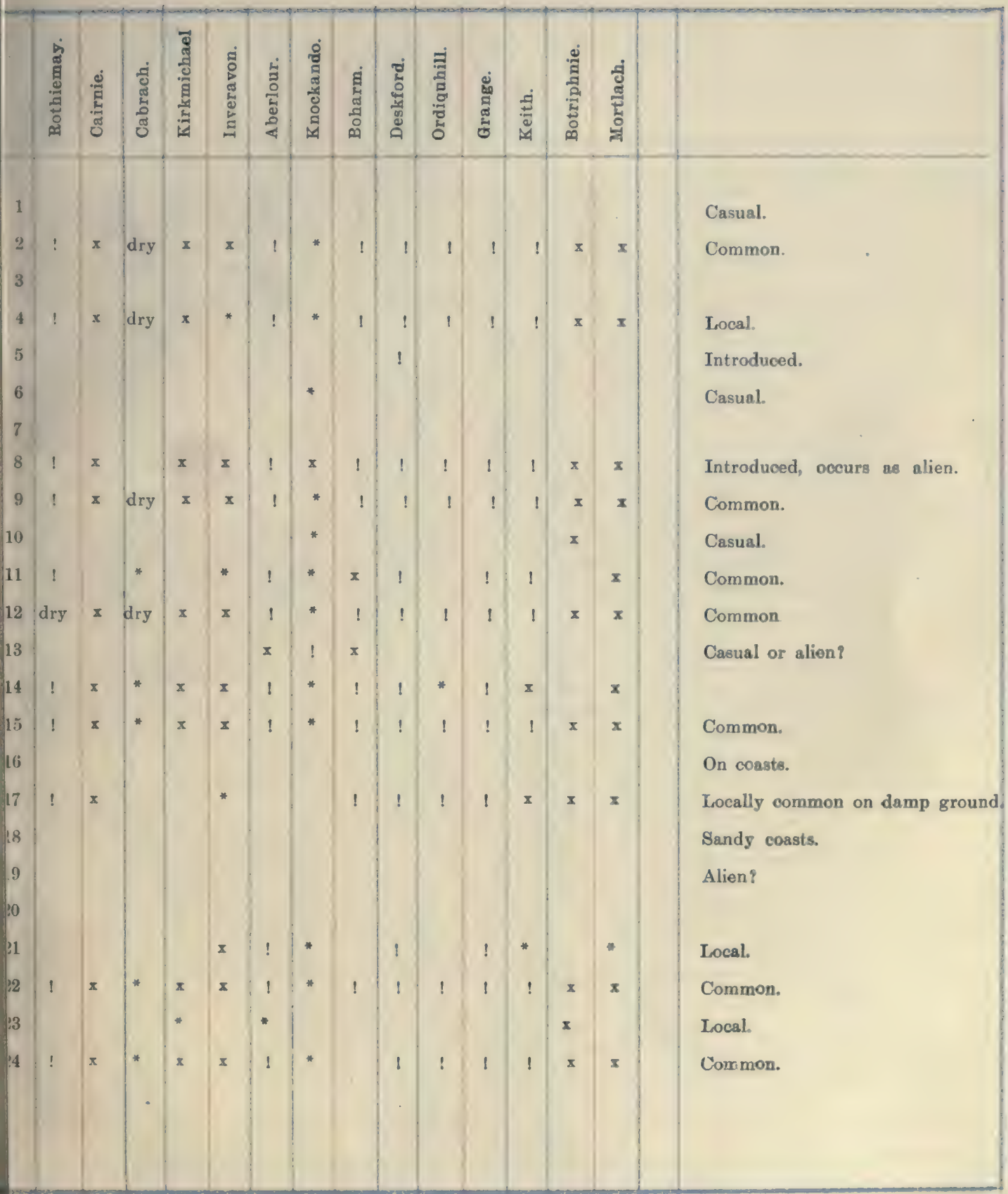




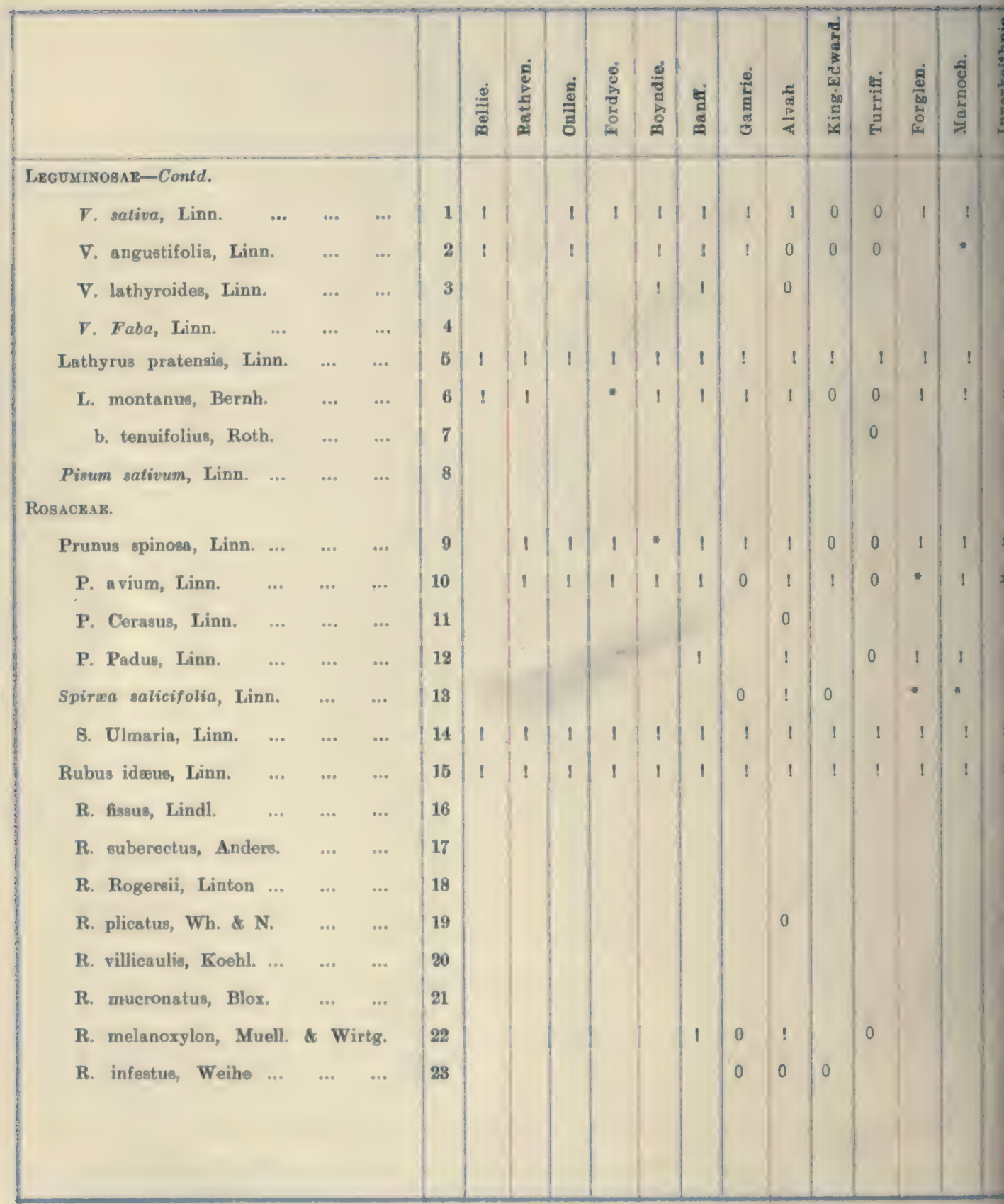




\begin{tabular}{|c|c|c|c|c|c|c|c|c|c|c|c|c|c|}
\hline हैं & 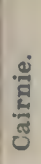 & गु & 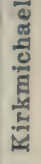 & 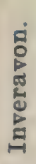 & 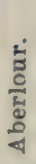 & 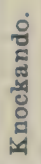 & 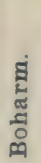 & 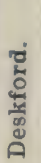 & 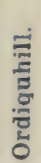 & हू & $\frac{j}{0}$ & 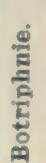 & 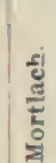 \\
\hline
\end{tabular}

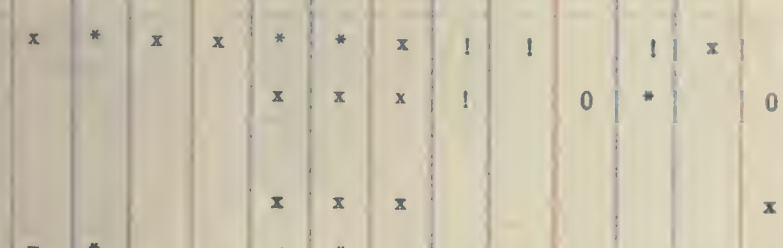

4

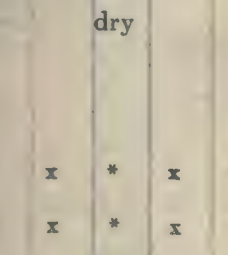

4
5
3
3
3
3
3
3 


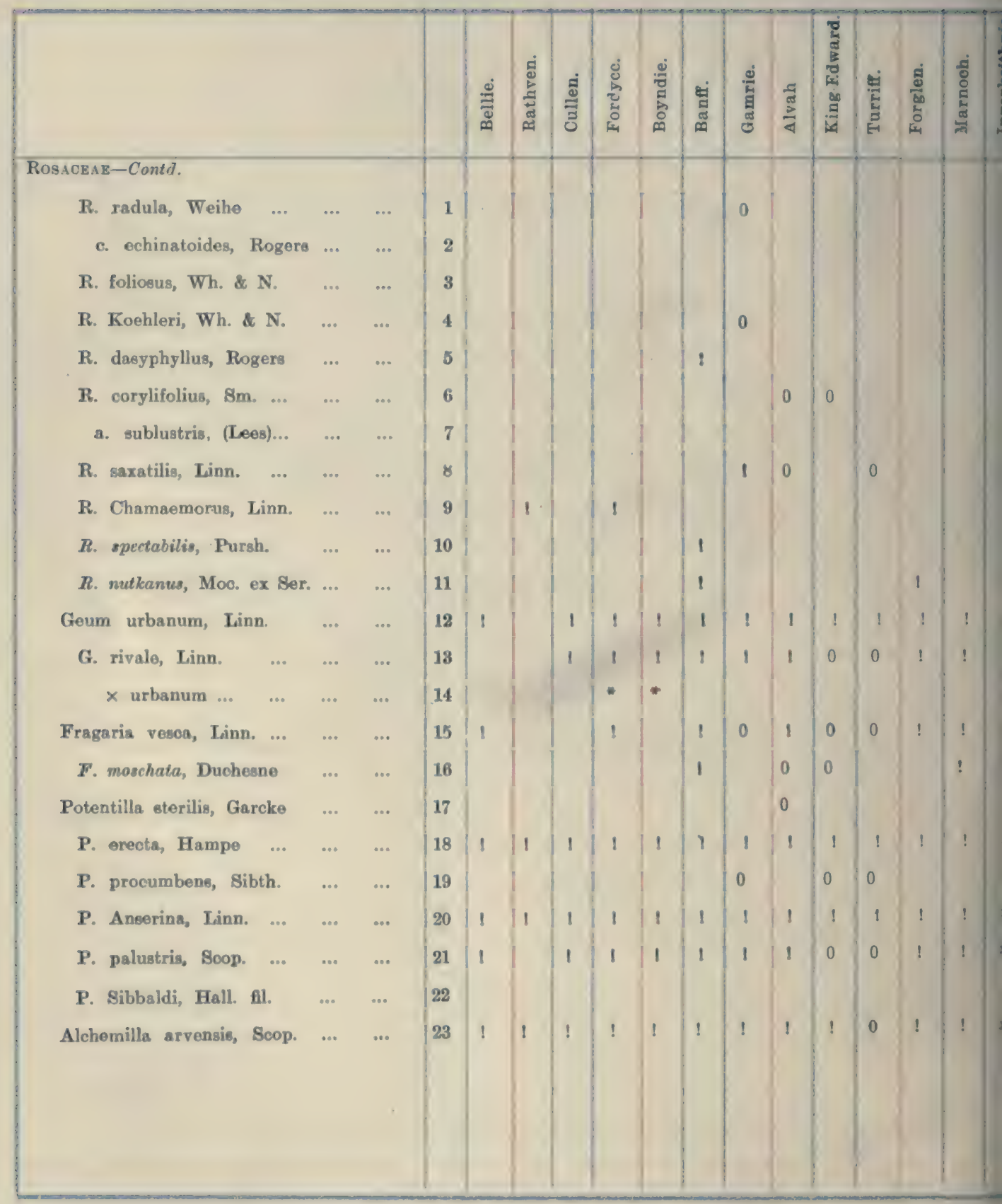




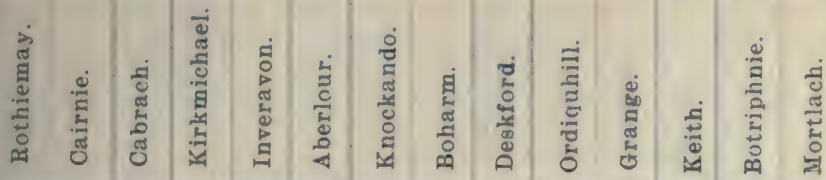

1

2

3

4

5

6

7

8

9

0

1

2

3 4

6

7

5

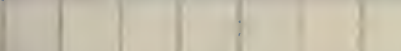




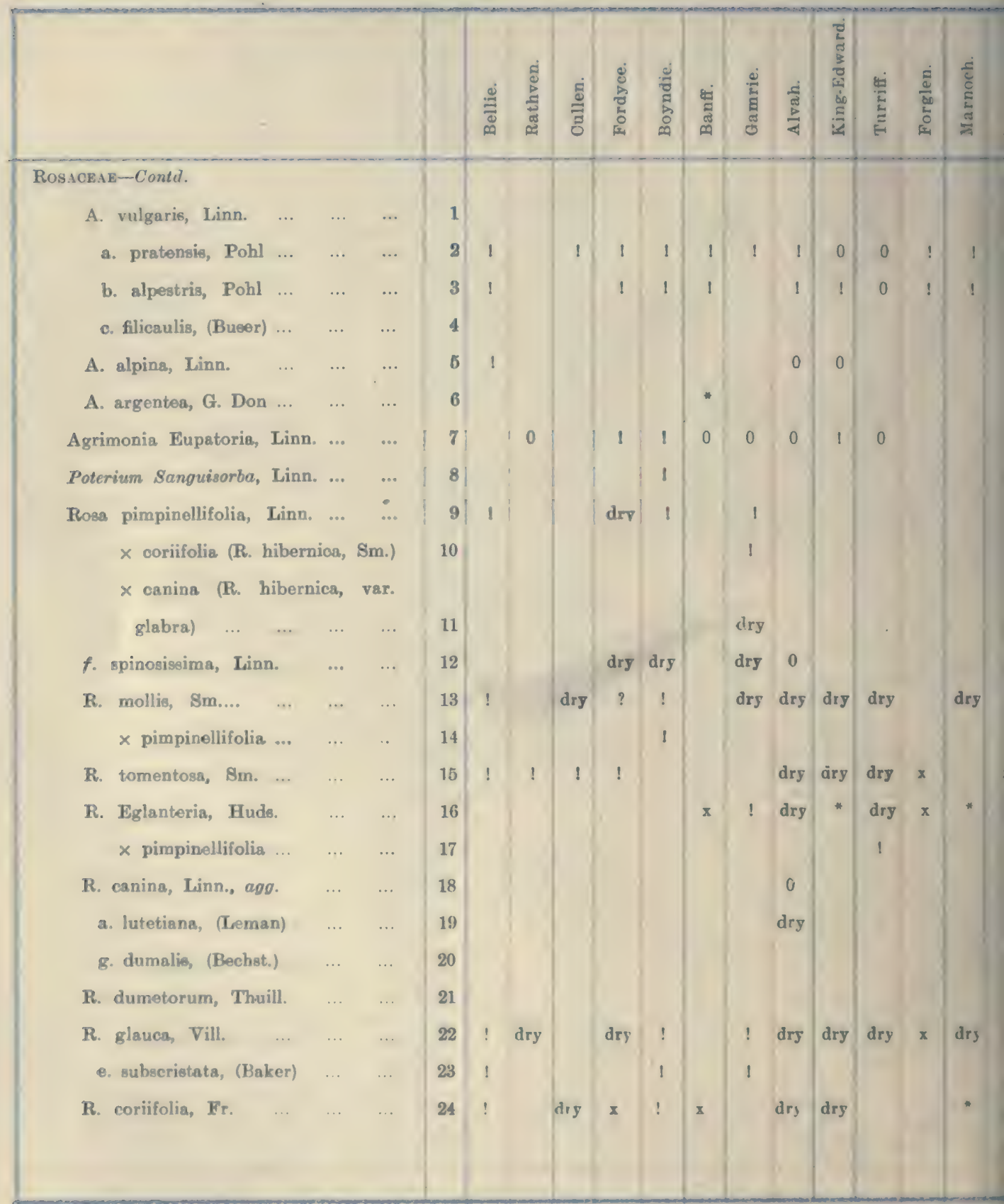




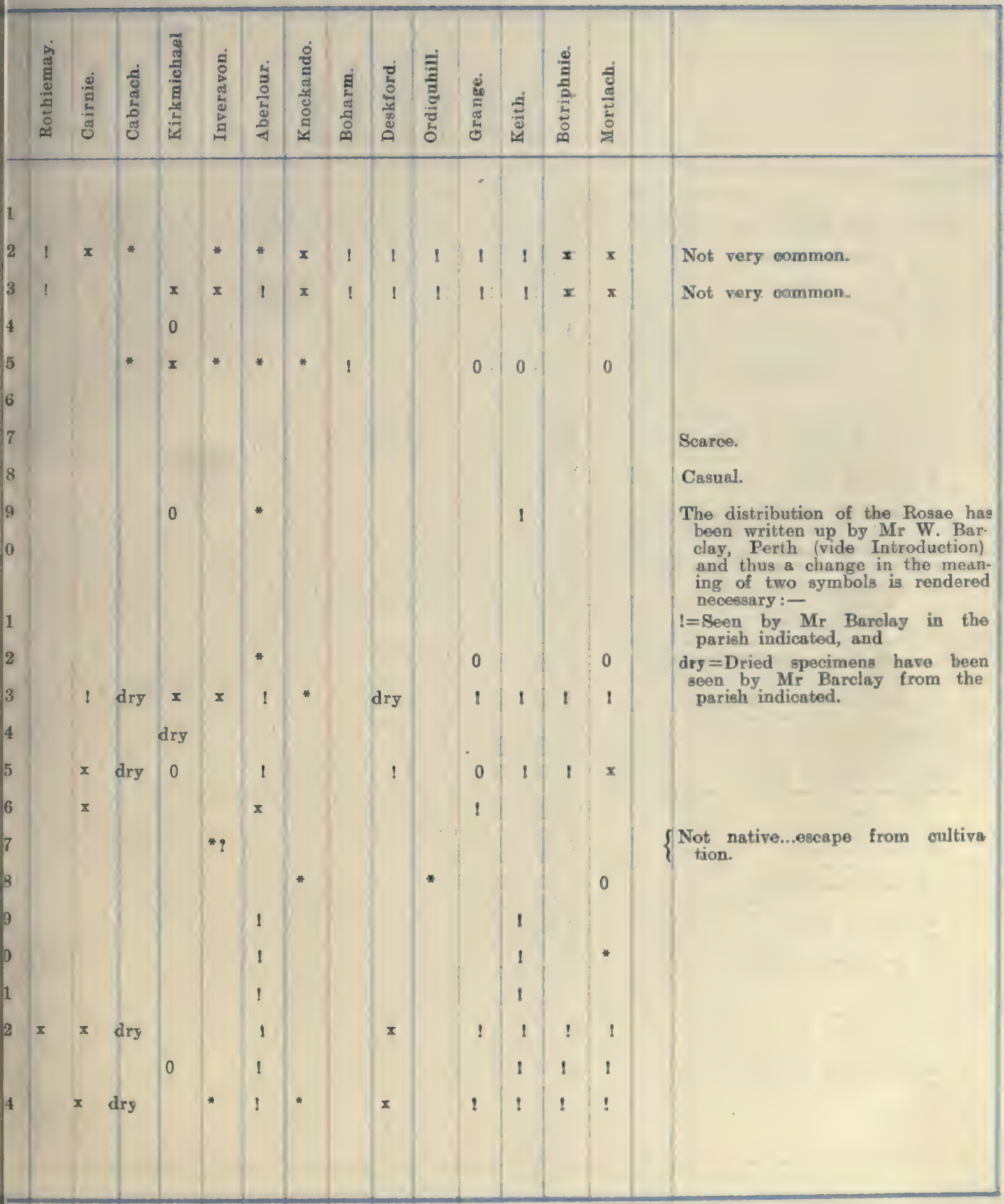




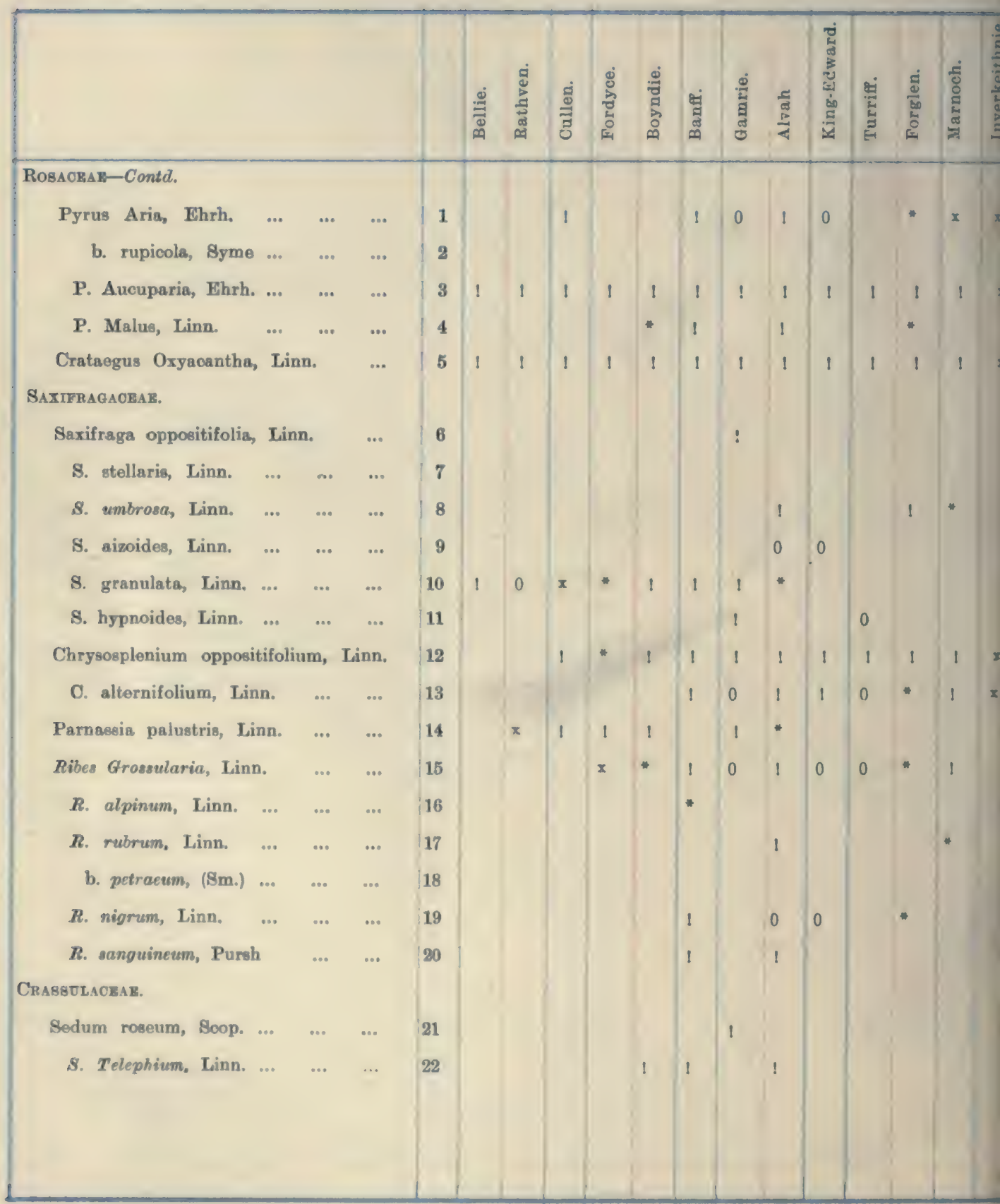




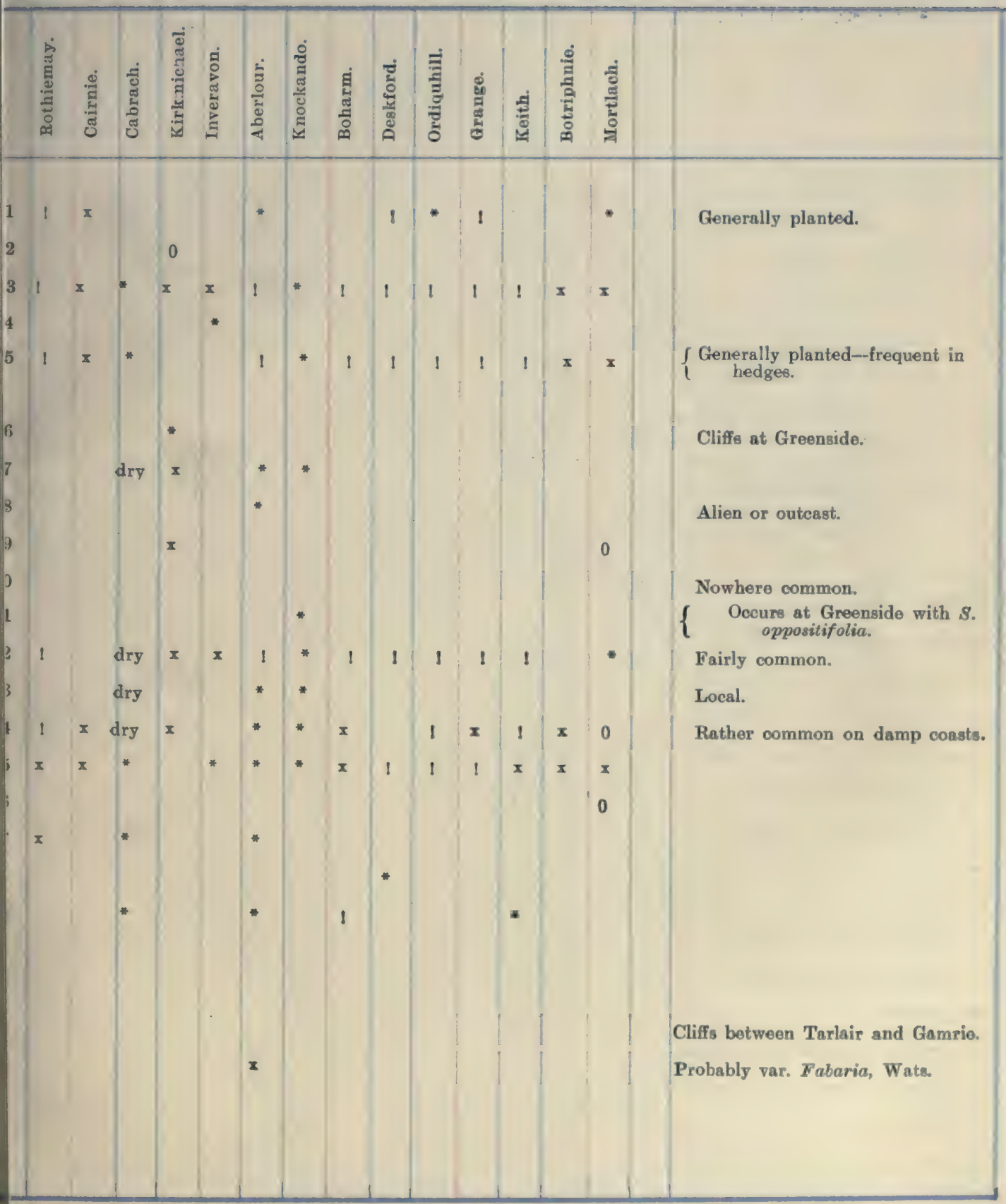




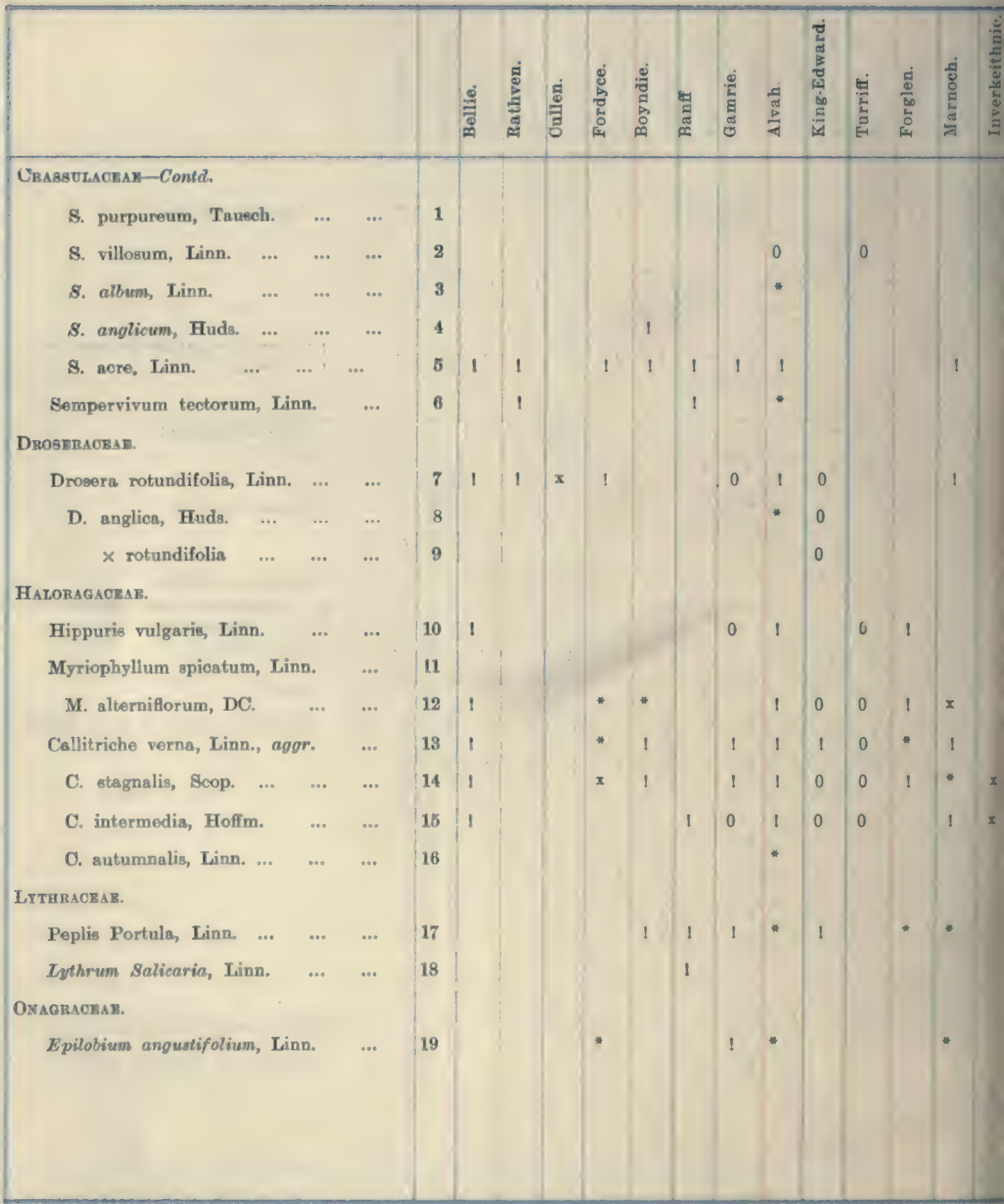




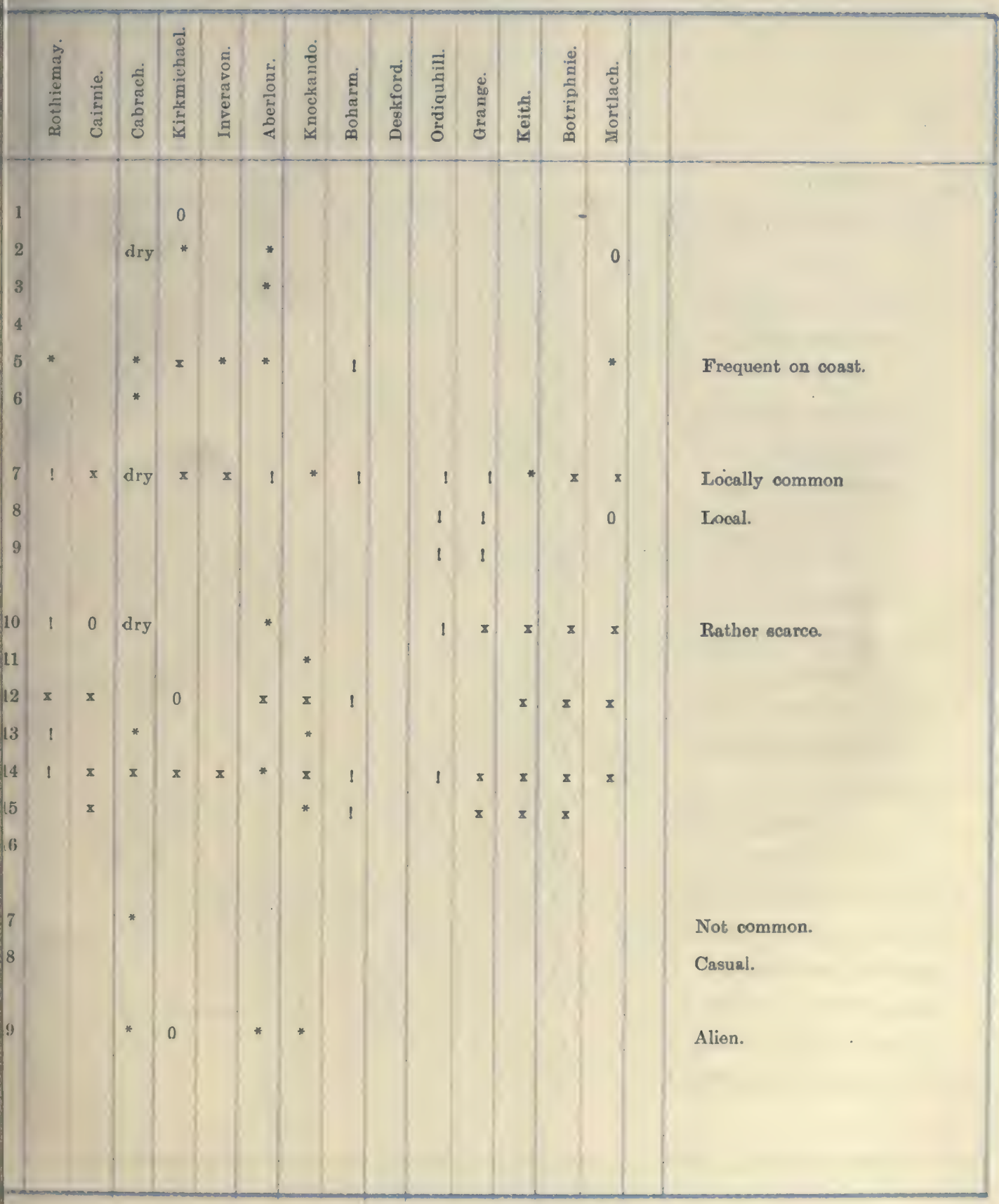




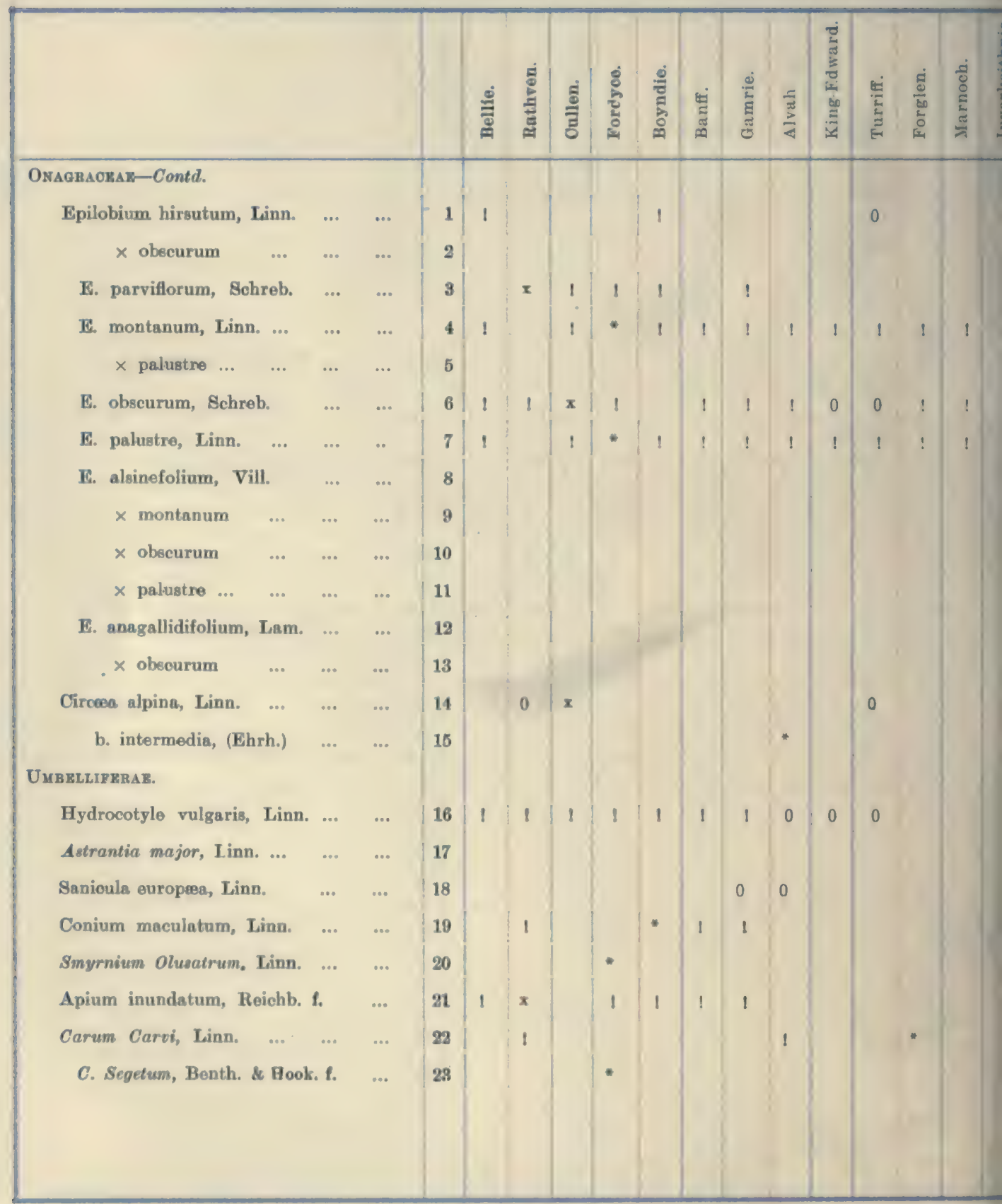




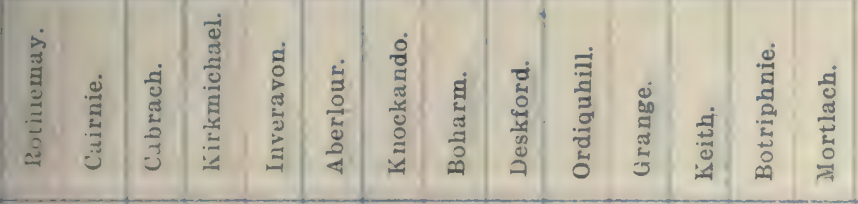

Local.

Local.

Rather common.

Ditches and marshy ground.

Ditches and marshy ground.

Rare.

Locally plentiful.

Local.

Denizen.

Local.

Alien.

Casual-I have seen no specimen. 


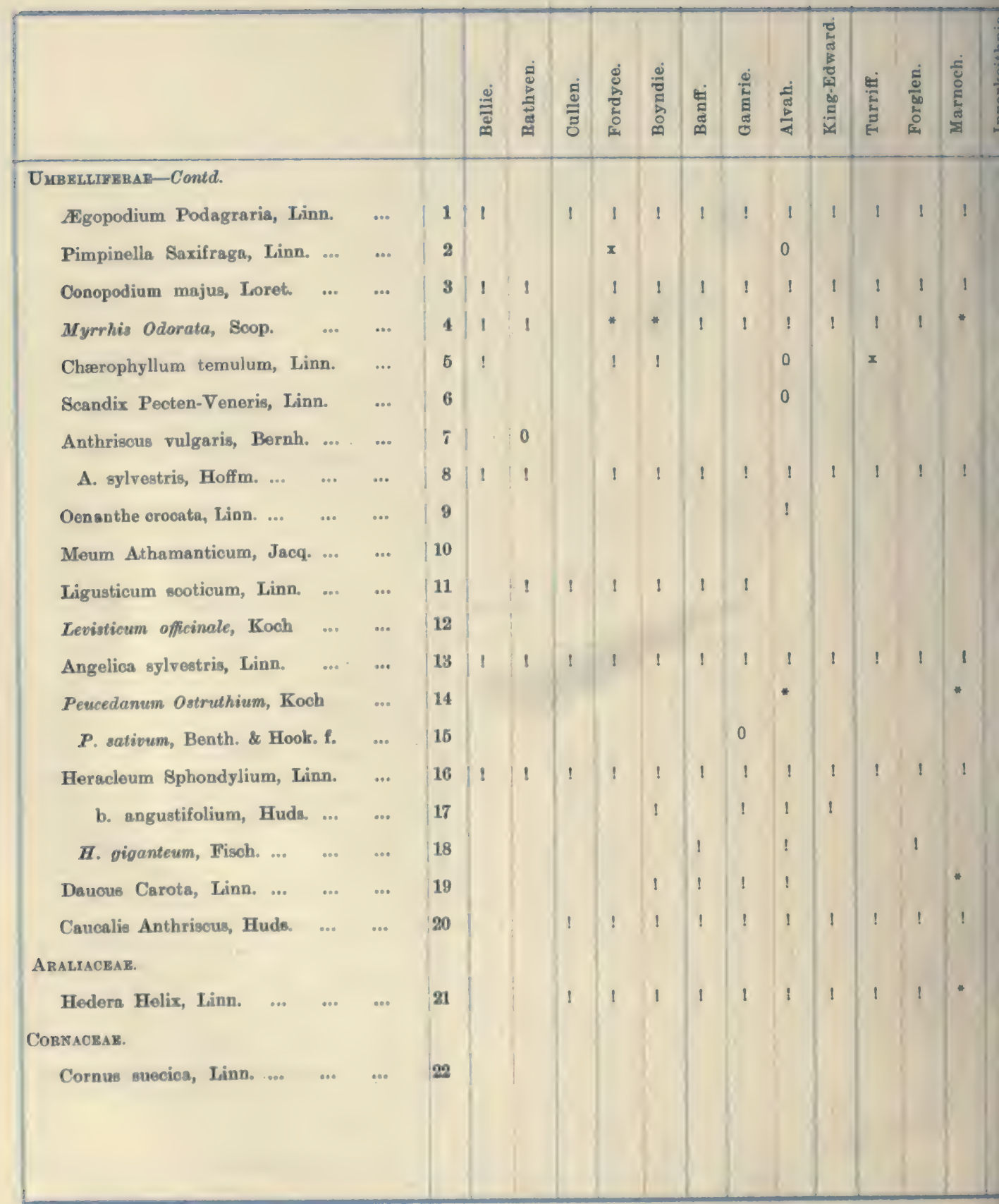




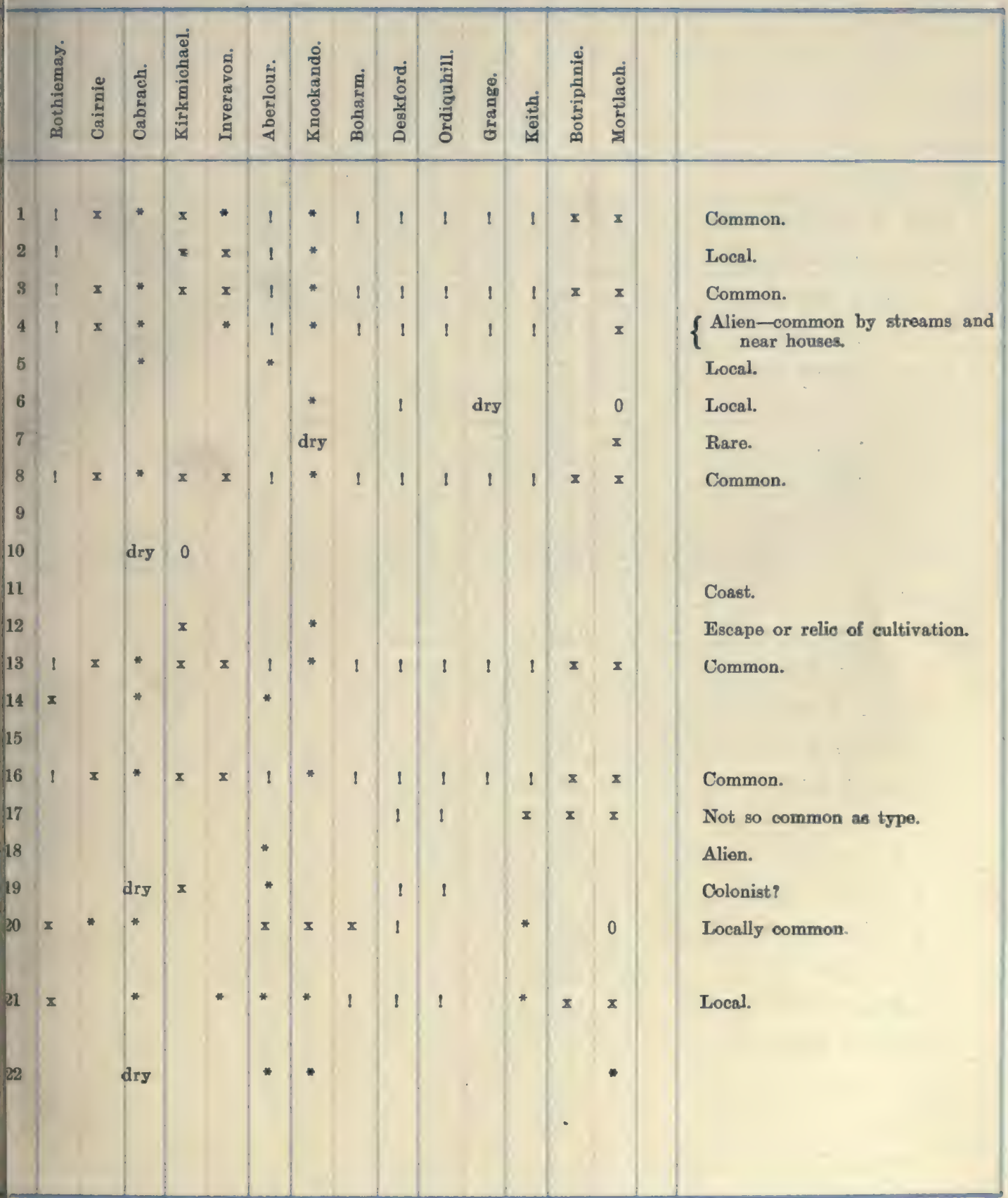




\section{@}

\section{Caprifoliagear.}

Adoxa Moschatellina, Linn. ..

Sambucus nigra, Linn. ..

S. Ebulus, Linn.

\$. racemosa, Linn.

Viburnum Opulus, Linn.

V. Lantana, Iinn

Linnea borealis, Lunn. ...

Lonioera Periclymenum, Linn.

L. Xylosteum, Linn.

Symphoricarpus racemosus, Michx. ... Rubtackaz.

Galium boreale, Linn.

G. Cruoiata, Scop.

G. verum, Iinn.

G. Mollugo, Linn.

G. saxatile, Linn.

G. asperum, Schreb. ...

G. palustre, Linn.

\begin{tabular}{llll|l} 
b. elongatum, (Presl.) & $\ldots$ & $\ldots$ & 18
\end{tabular}

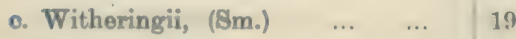

G. uliginosum, Linn.

G. Aparine, Linn.

G. tricorne, Stokes
1

2

8

4

5

6

700

8

9

10

11

12

13

14

15

16

17

$19 \quad$

20

21

22 


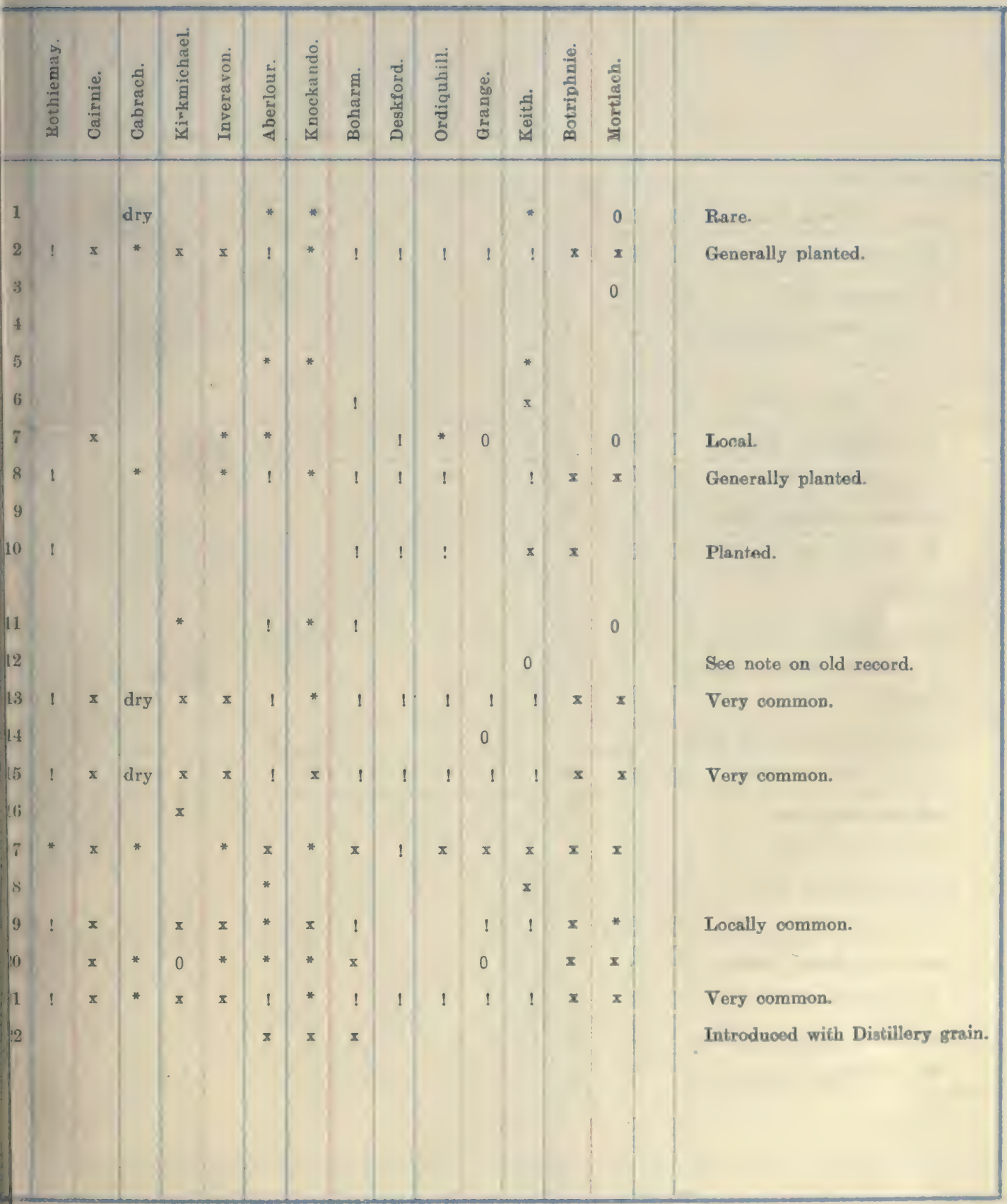




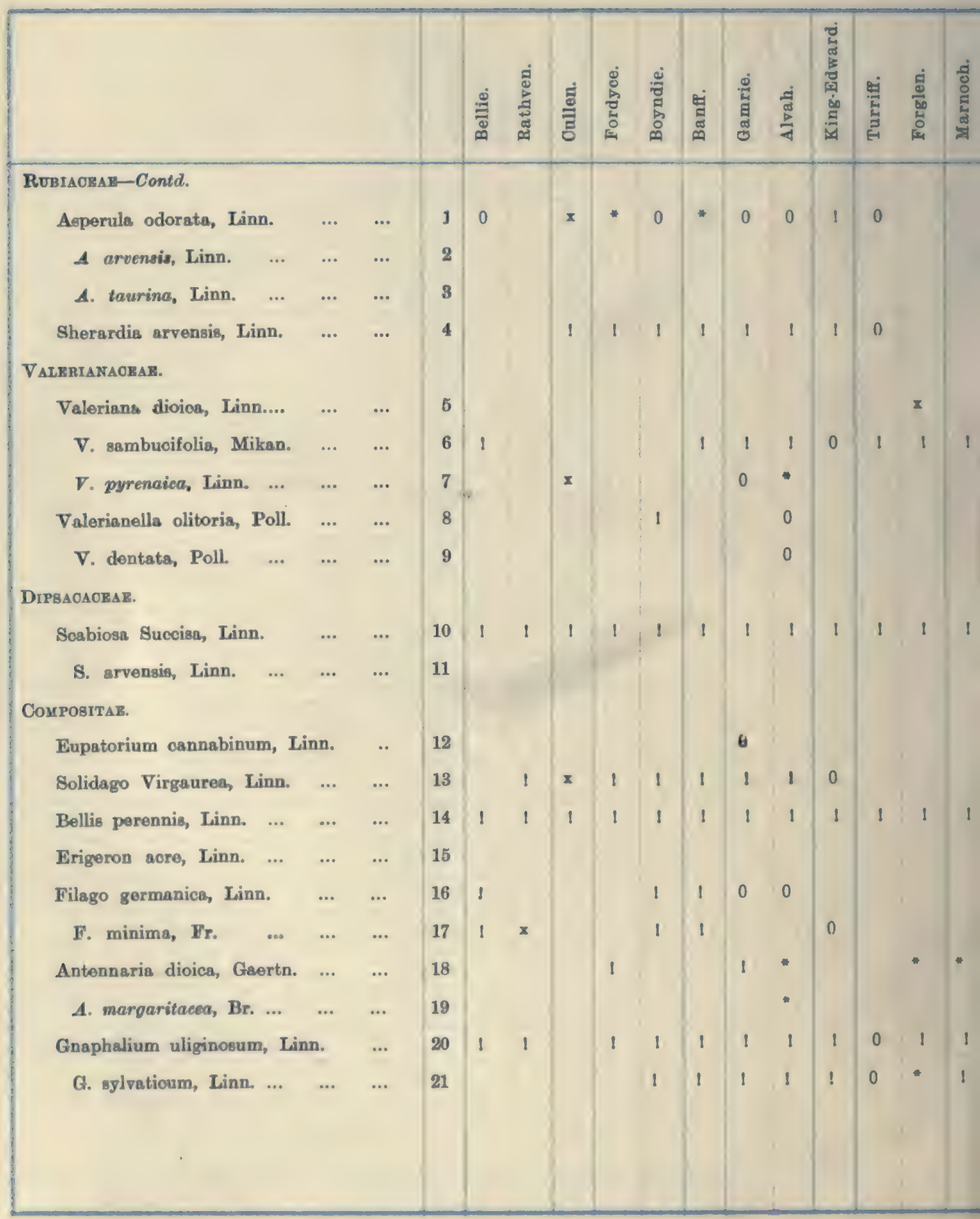



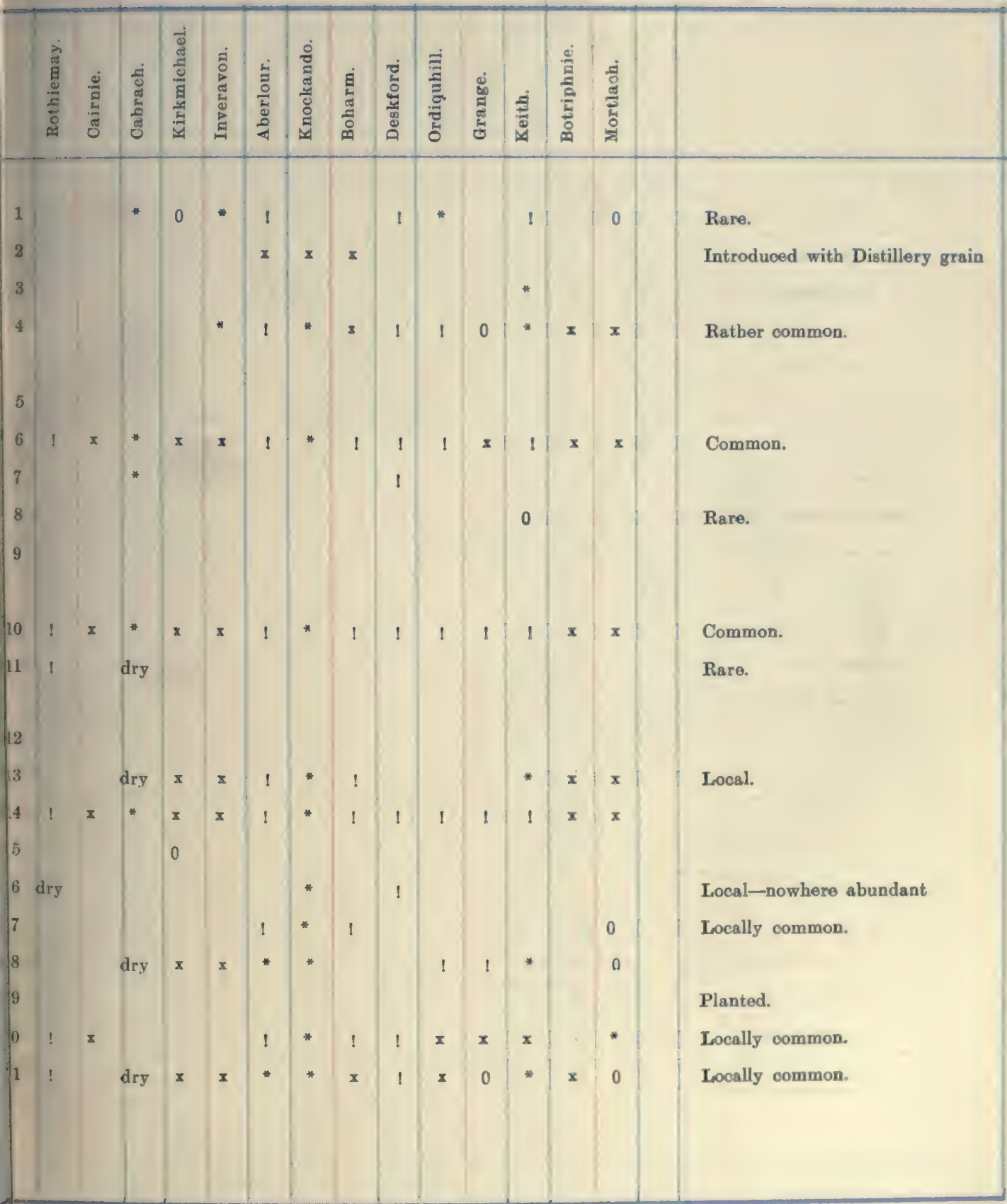


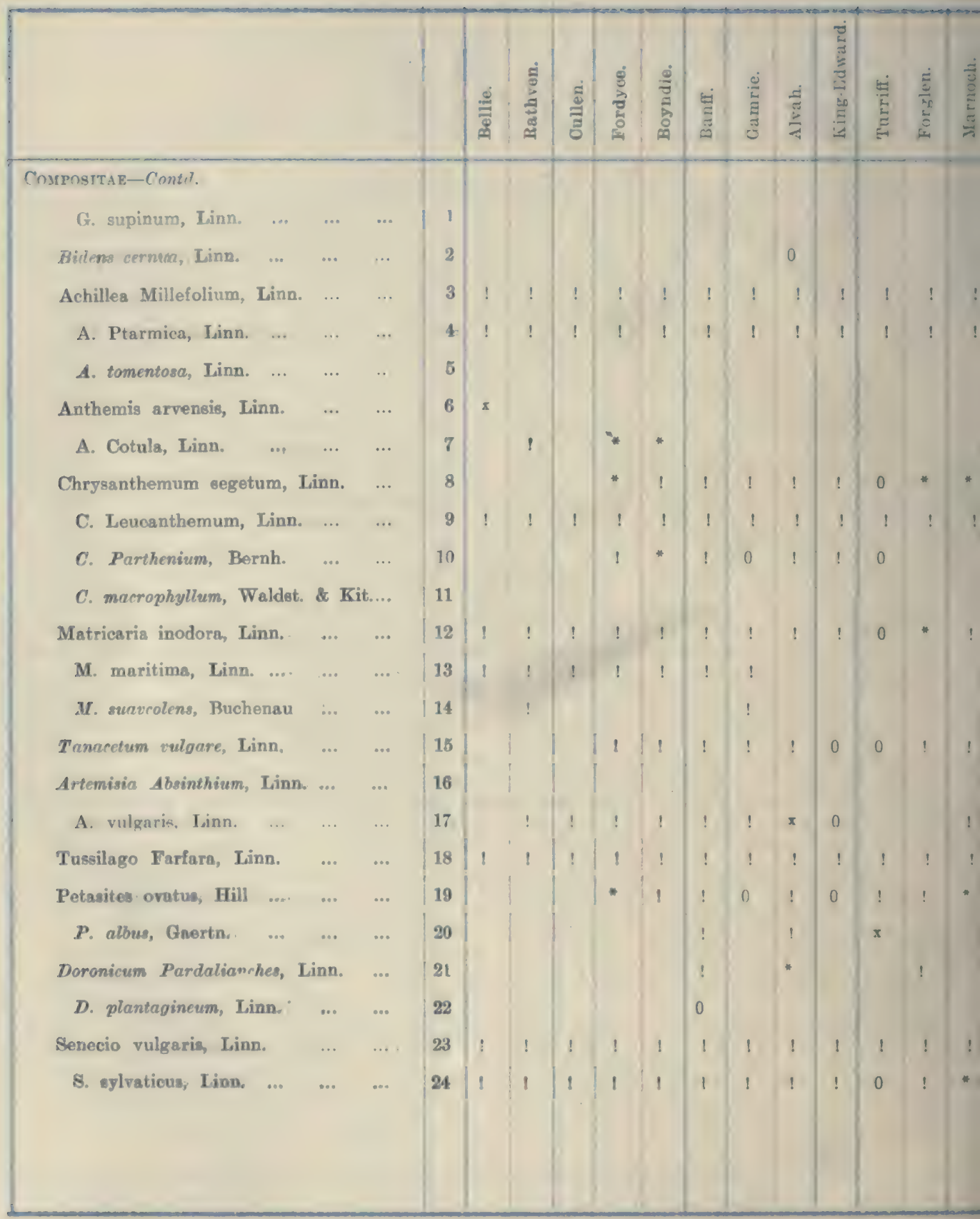




\section{$|11 \| 1| 111 \mid 111$}

Very common.

\section{Common.}

Colonist.

Common.

Alien.

Common.

Coast.

Denizen.

Alien.

Local.

Very common.

Frequent by streams.

Alien.

Alien.

Very common

Common. 


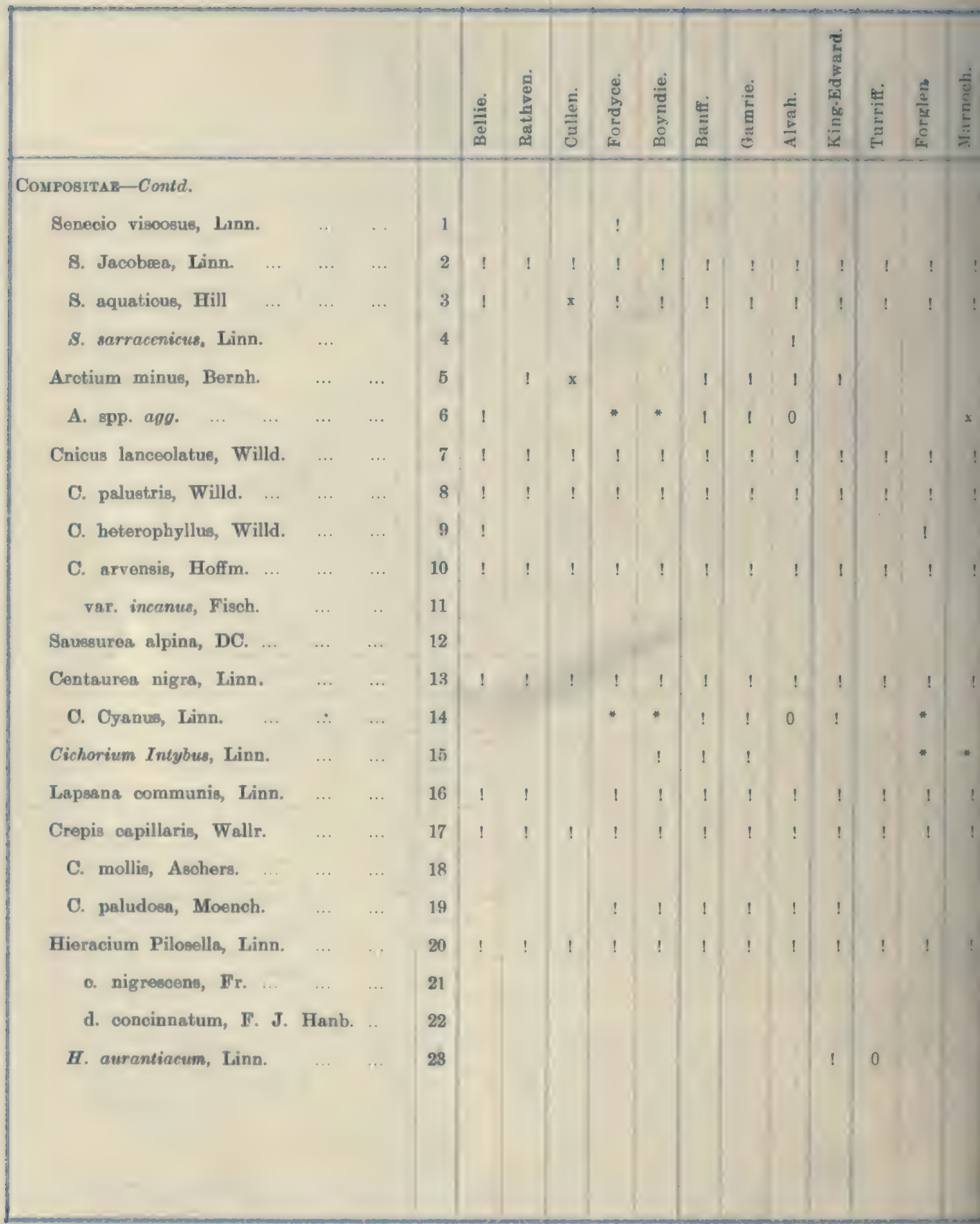




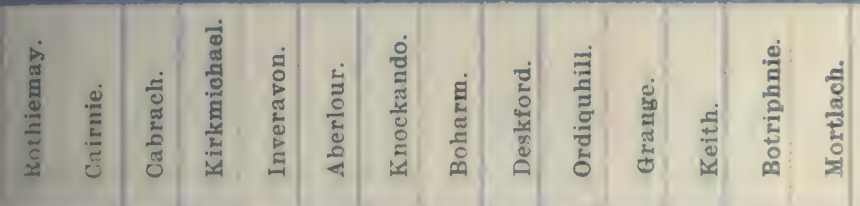

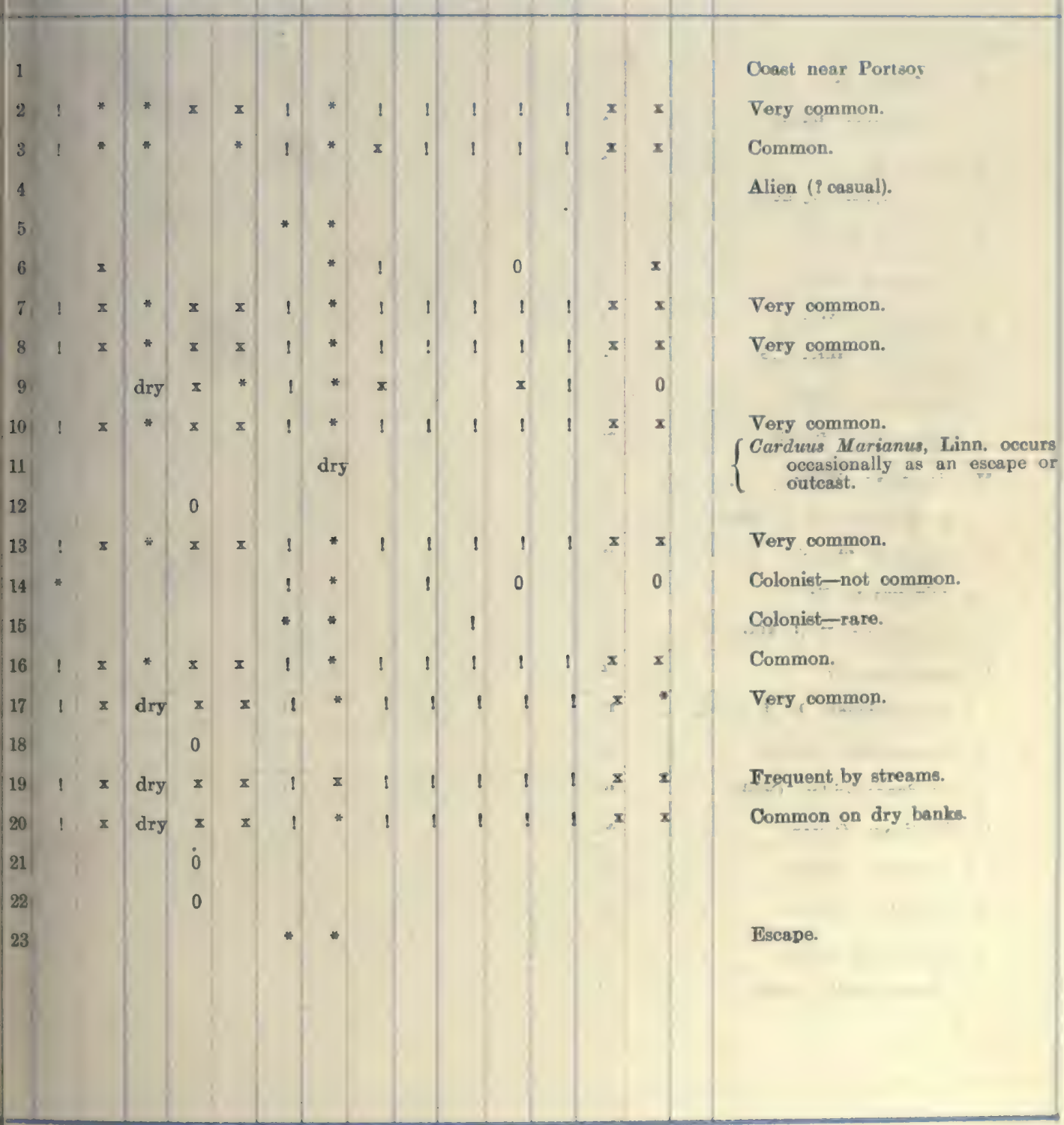




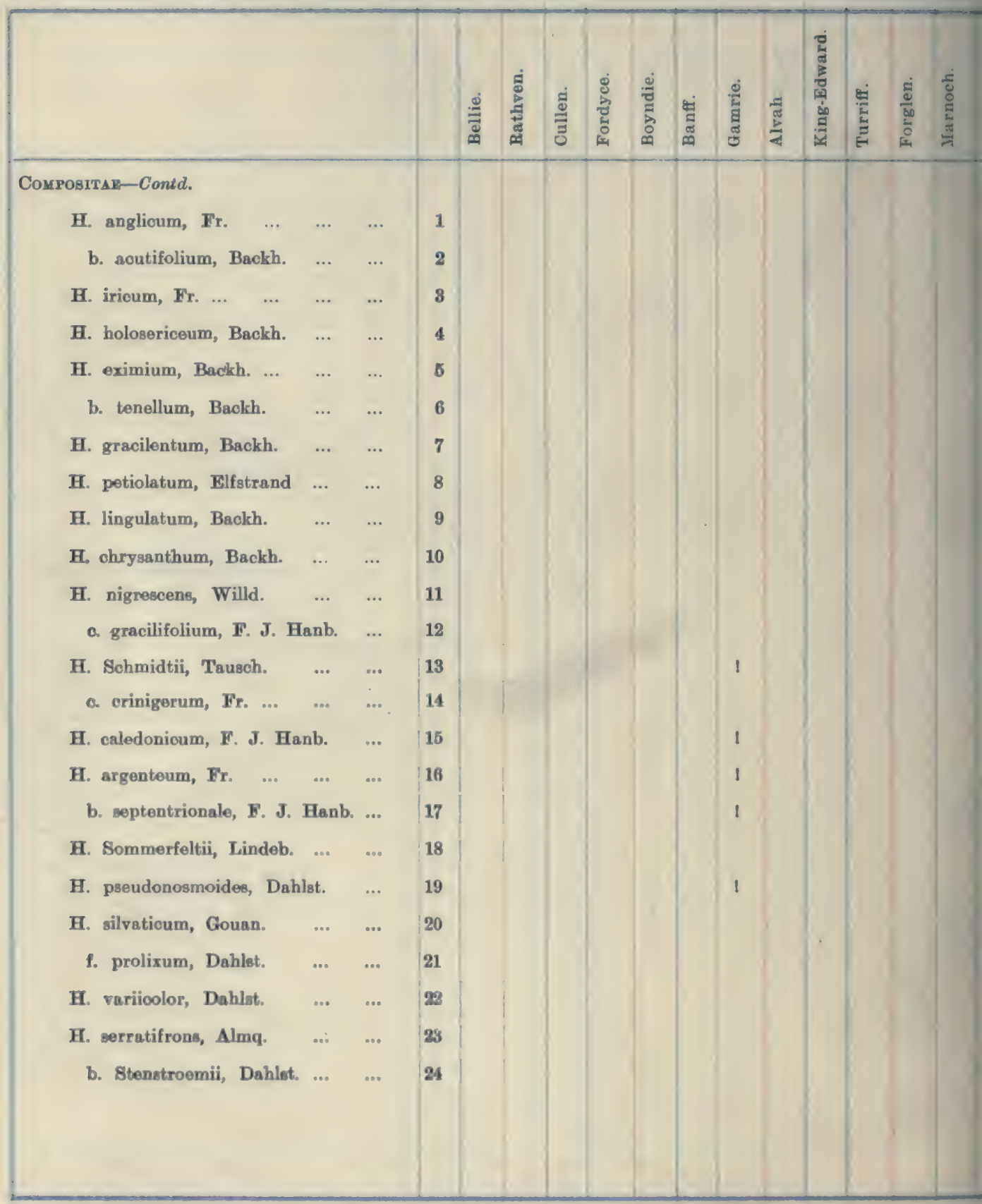




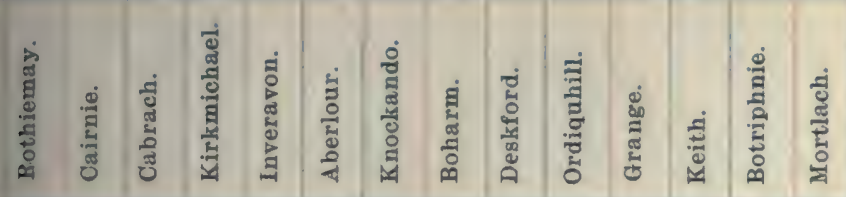

Coast in Gamrio-rare.

Coast in Gamrie-rare. 


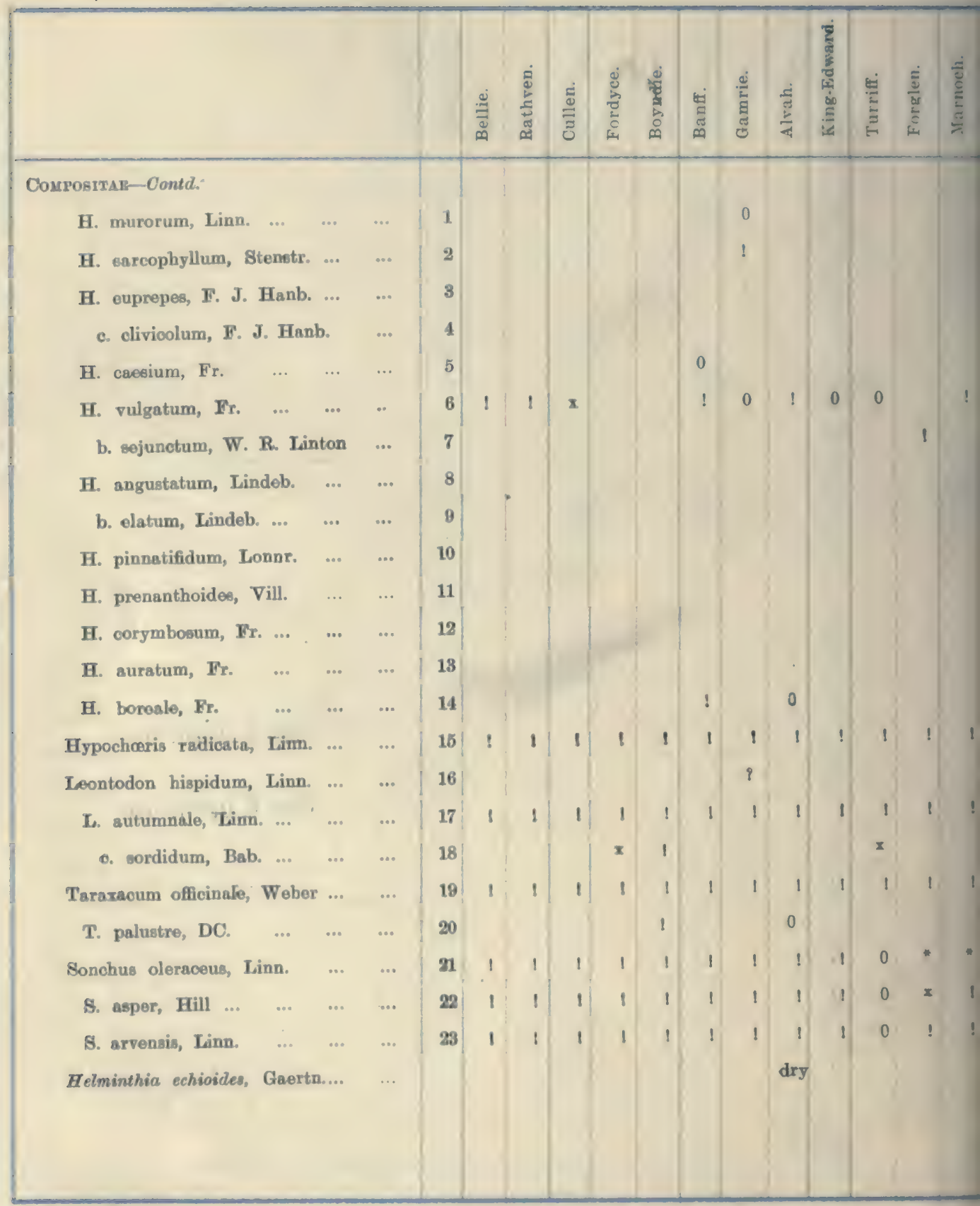




\section{1}

Frequent.

Very common.

Doubtful record.

Very common.

Very common.

Local.

Fields and waste places.

Fields and waste places.

Fields and waste places.

Casual. 


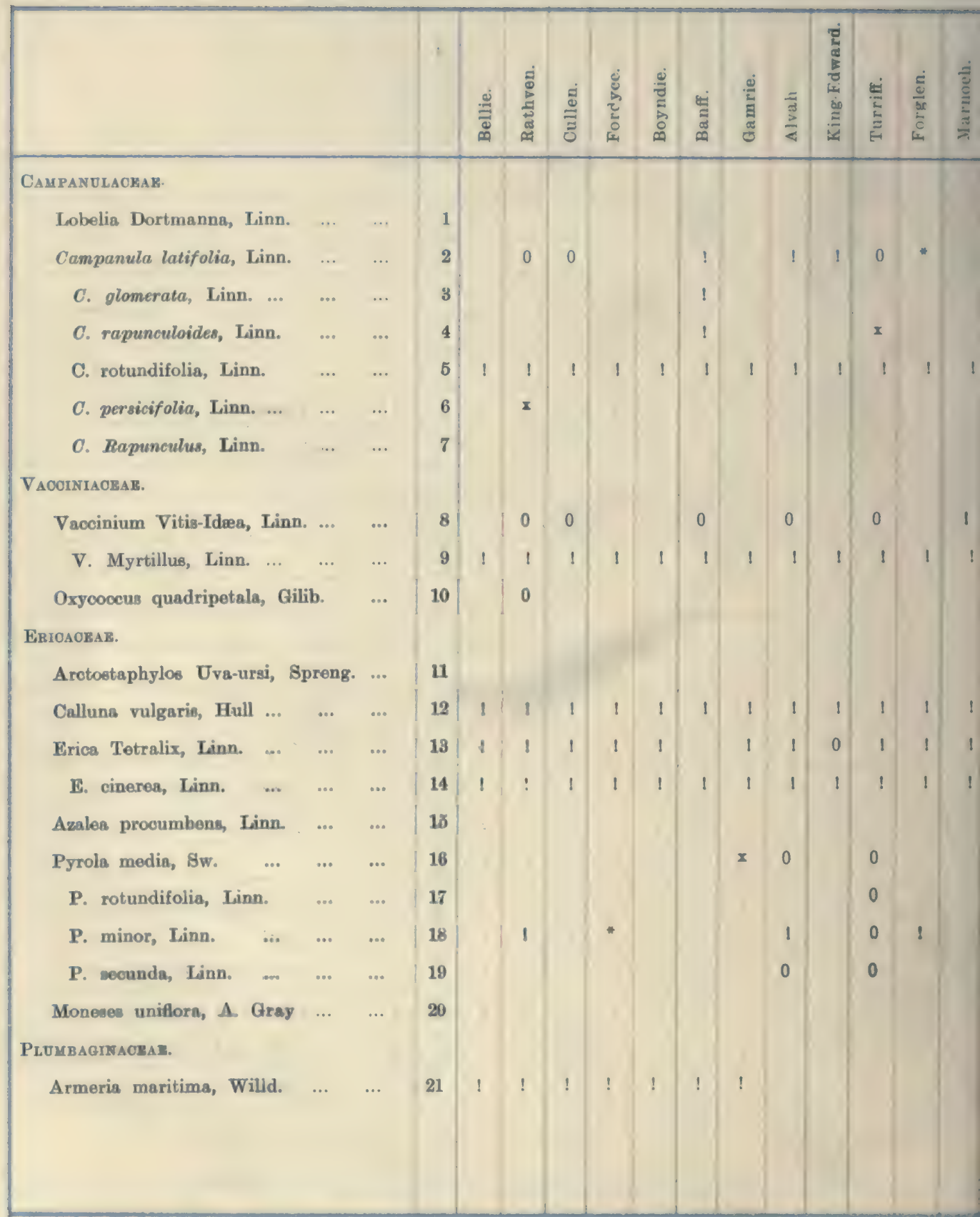




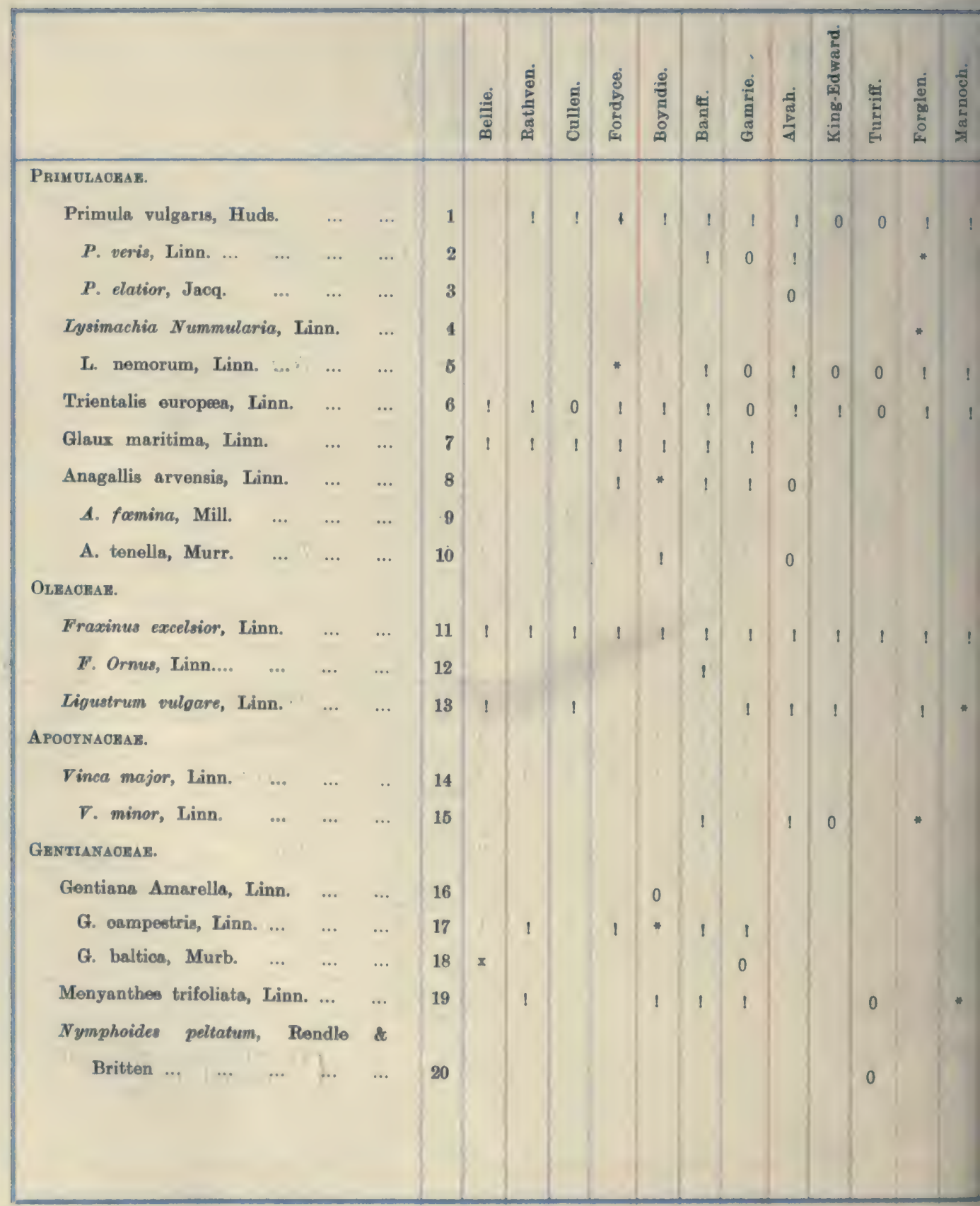




\section{4}

Alien.

\{ Local, especially in damp shady \{ woods.

Local, in woods and on moors.

Const.

Rather scarce.

Rare.

Generally planted.

Planted in Duff House Policies.

Often planted in hedges.

\section{Alien.}

Coften confused-a thorough revision of the forms in the $\mathbf{N}$. of Scotland is necessary.

Local.

Introduced. 


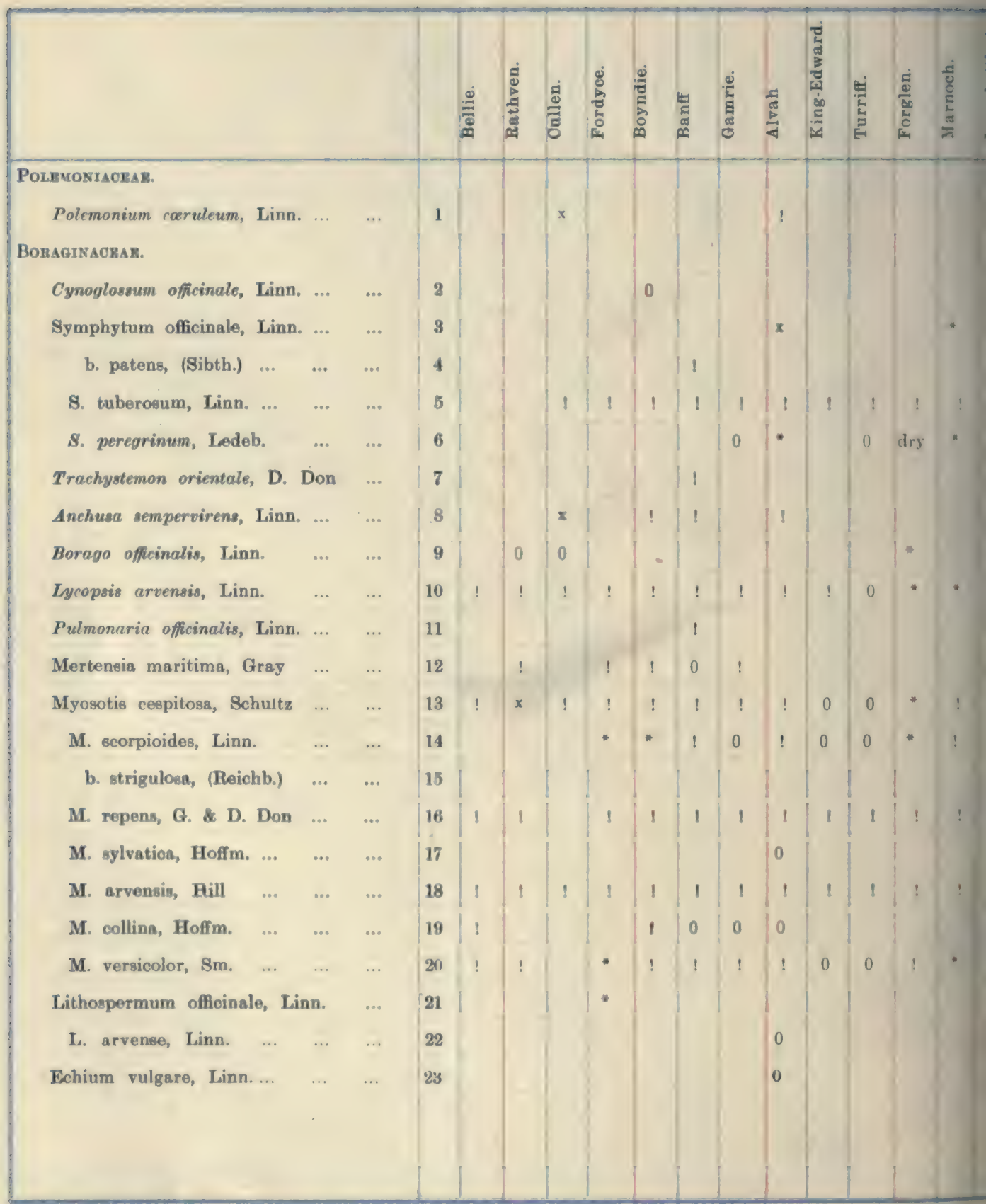




\begin{tabular}{|c|c|c|c|c|c|c|c|c|c|c|c|c|c|}
\hline :ुँ & . & 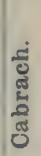 & $\frac{\bar{d}}{\frac{3}{3}}$ & 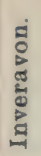 & $\begin{array}{l}\frac{3}{3} \\
\frac{0}{2} \\
\frac{3}{0} \\
\end{array}$ & 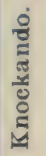 & 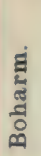 & 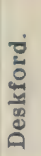 & 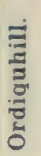 & 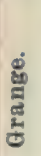 & 递 & 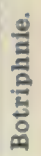 & 혈 \\
\hline
\end{tabular}

Scarce.

Locally common.

Alien.

Alien.

Colonist.

Alien in Duff House Policies.

Coast

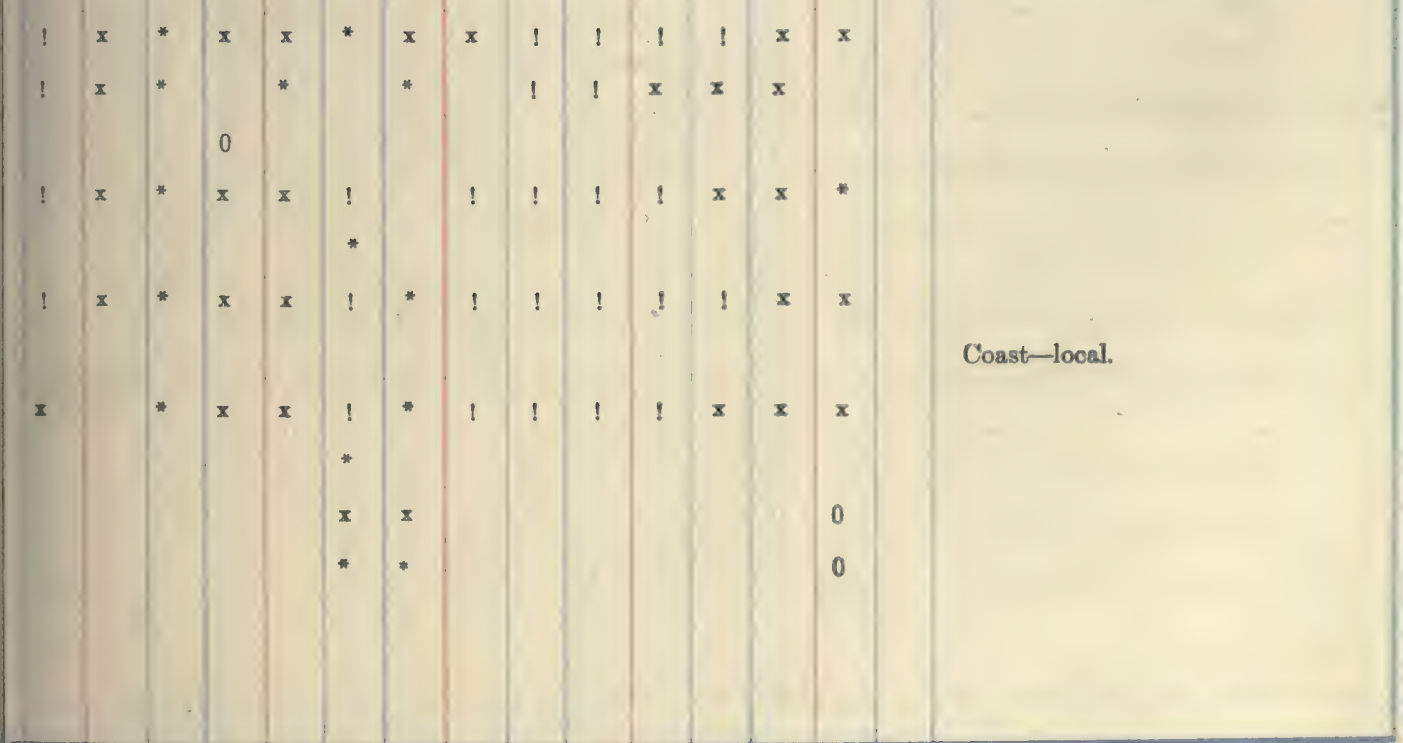




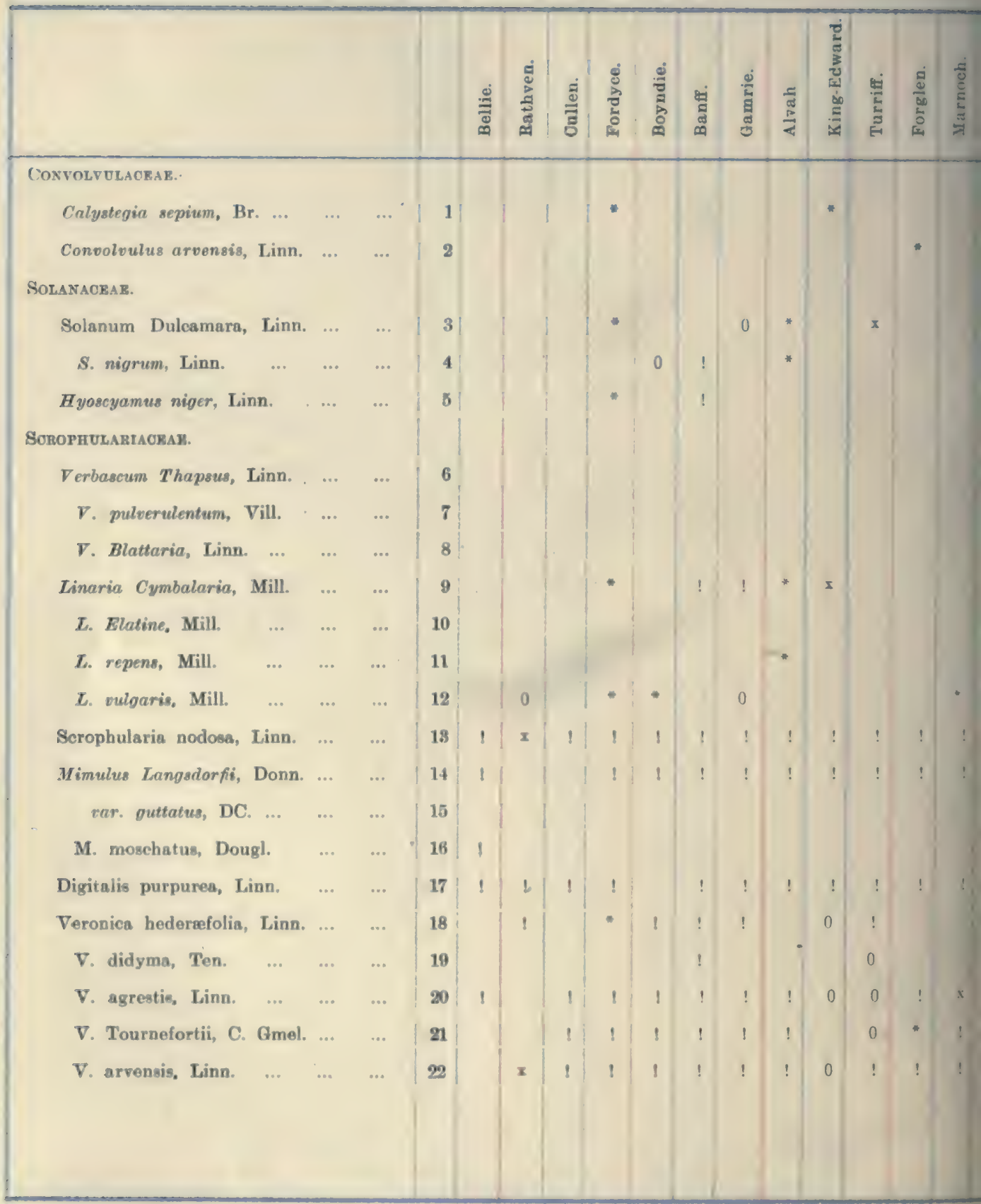



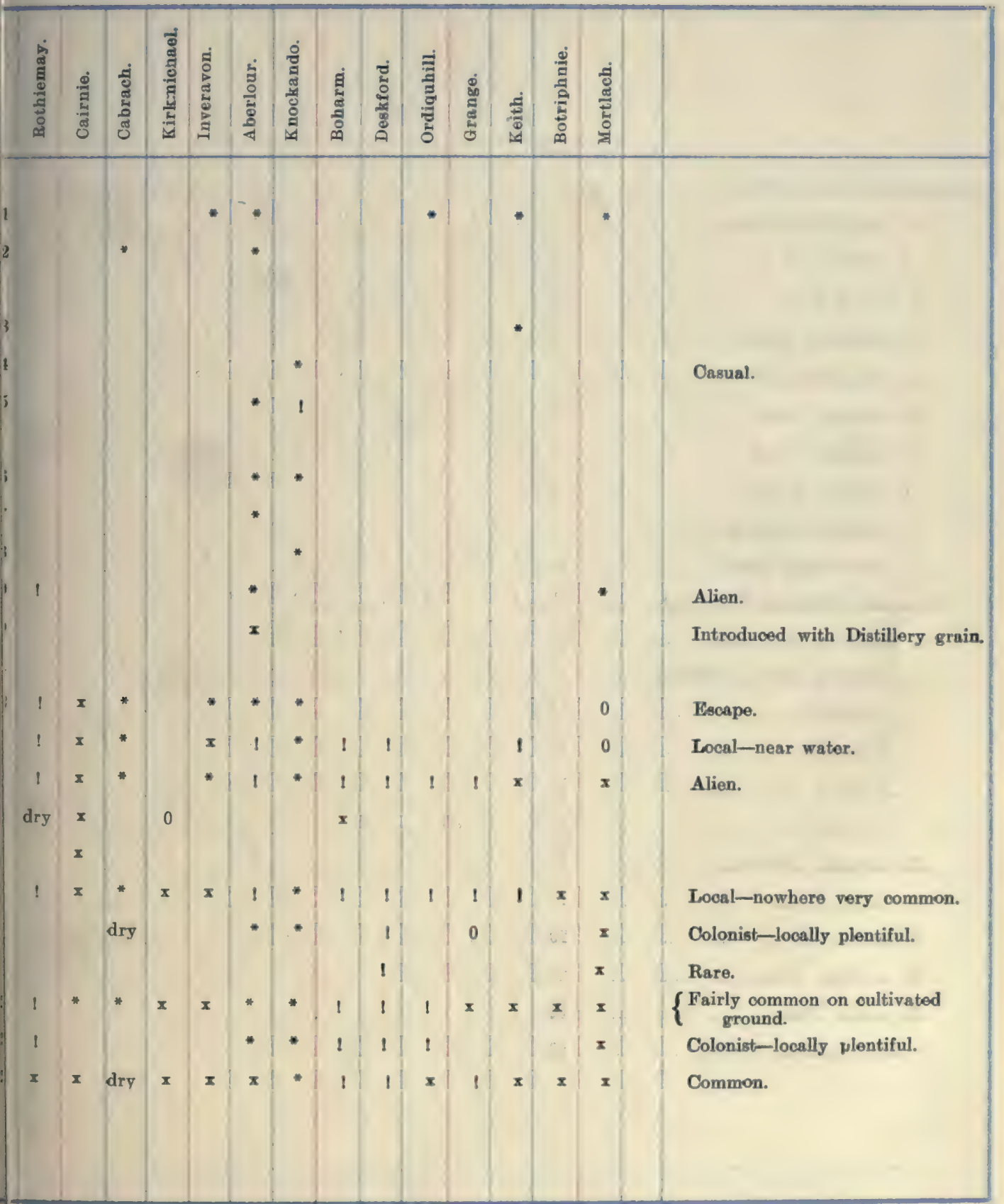


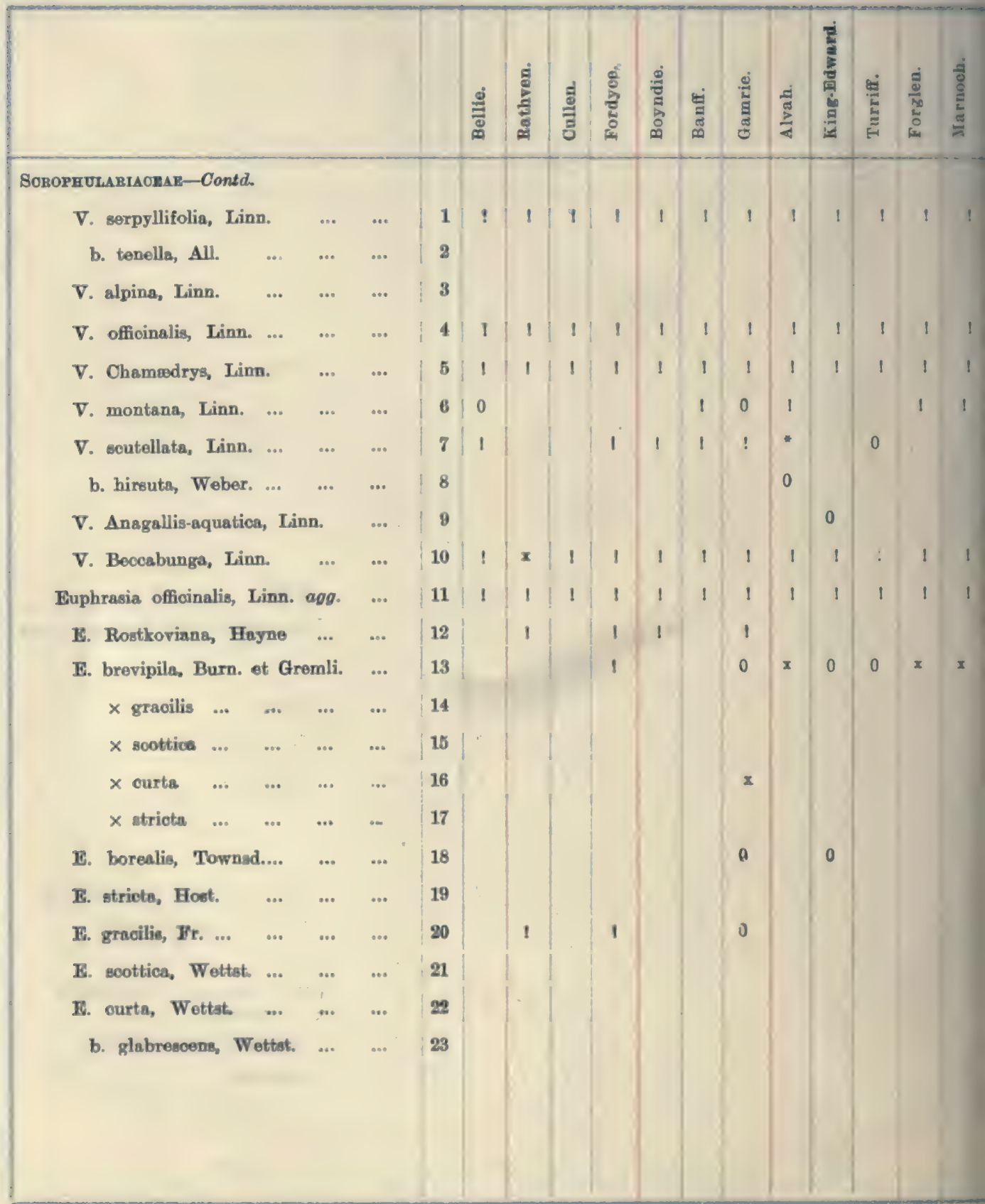




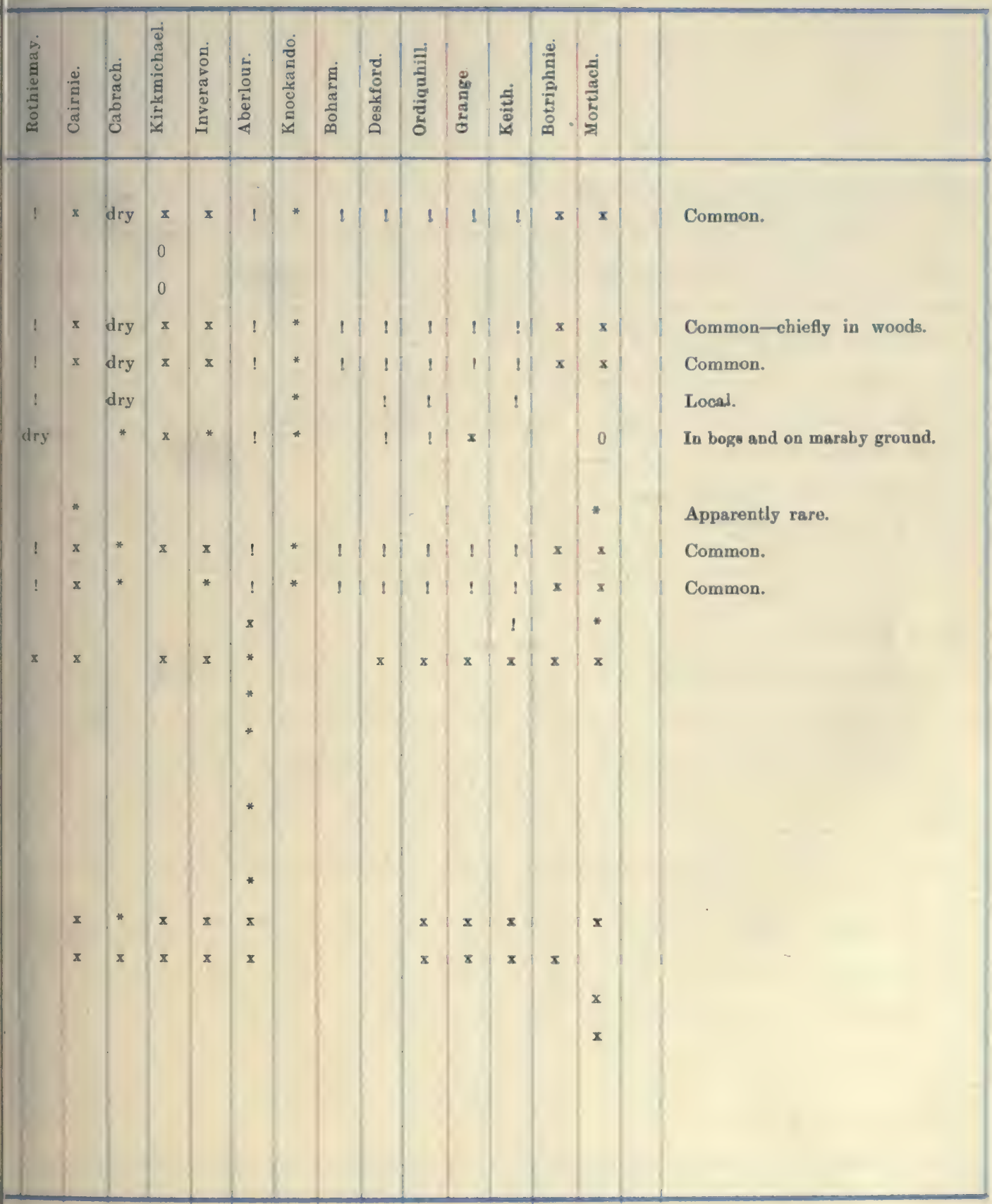




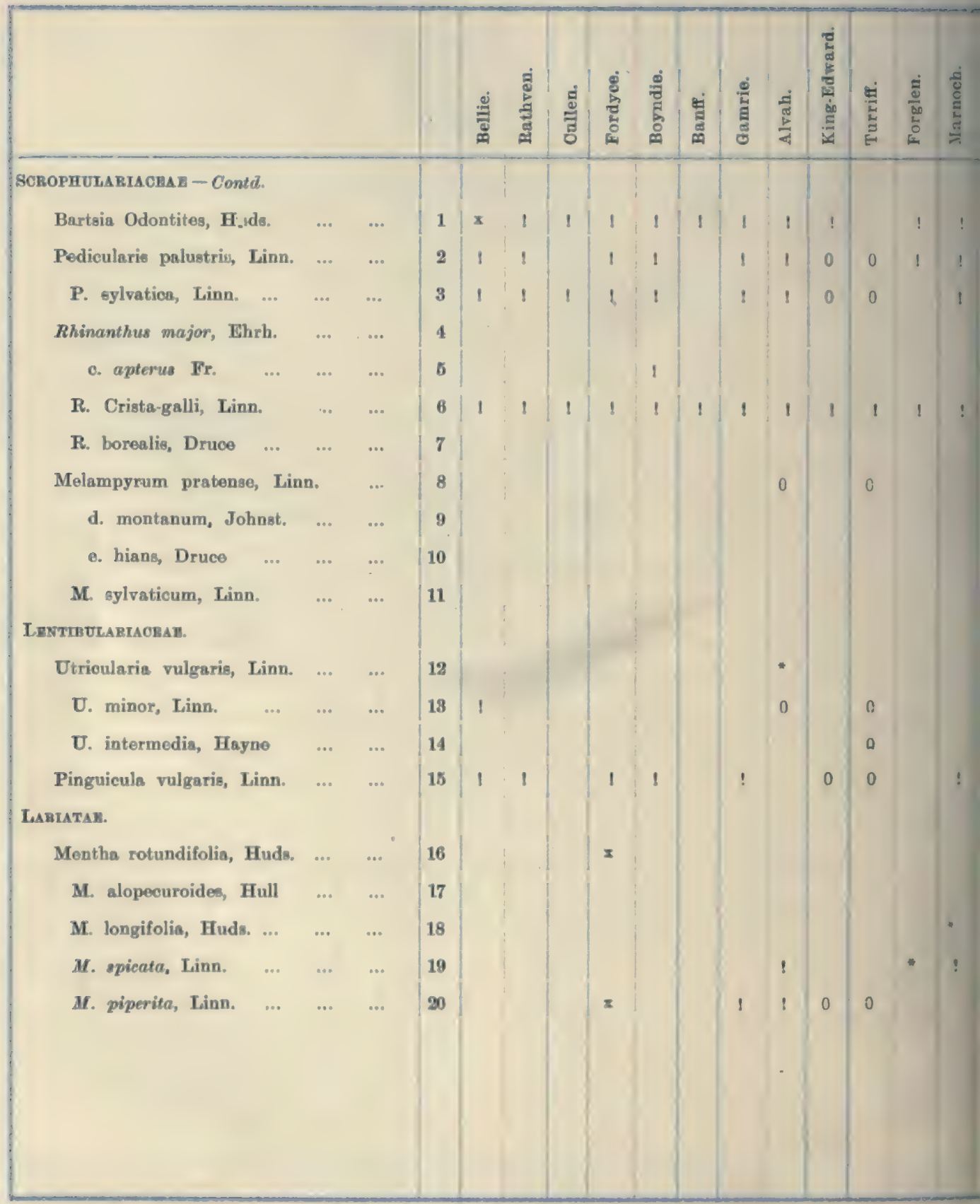




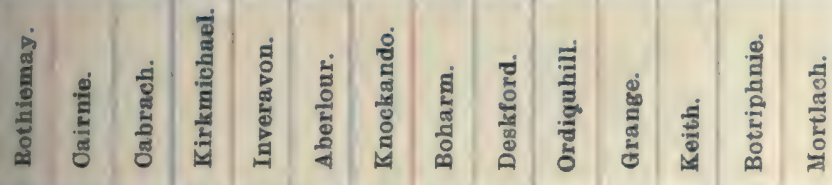

On damp ground and by roadsides.

Casual.

Common.

Common locally. 
Labiatak-Contd.

M. aquatica, Linn.

\begin{tabular}{lllll|l} 
a. hirsuta, $($ Huds.) & $\ldots$ & $\ldots$ & $\ldots$ & 2
\end{tabular}

\begin{tabular}{ll|l|l|l|} 
o. $\operatorname{citrata,}($ Ehrh.) & $\ldots$ & $\ldots$ & $\ldots$ & $\mathbf{3}$
\end{tabular}

$\times$ arvensis, Linn.

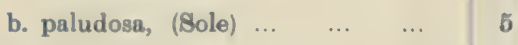

M. rubra, $\mathrm{Sm}$.

M. arvensis, Linn.

Origanum vulgare, Linn.

Thymus Serpyllum, Linn.

$\begin{array}{llll}\text { b. prostratum, Hornem. ... } & \ldots & 10\end{array}$

\begin{tabular}{llll|l} 
Clinopodium vulgare, Linn. ... & $\ldots$ & 11
\end{tabular}

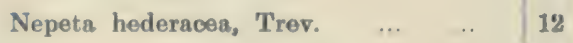

\begin{tabular}{lll|l} 
Scutellaria galerioulata, Linn. & $\ldots$ & 13
\end{tabular}

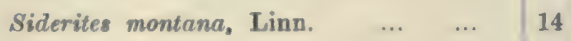

Prunella vulgaris, Linn.

Marrubium vulgare, Linn.

Stachys palustris, Linn. $\times$ sylvatica

8. sylvatica, Linn.

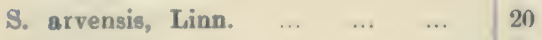

\begin{tabular}{llll|l} 
Galeopsis speciosa, Mill. & $\ldots$ & $\ldots$ & 21
\end{tabular}

G. Tetrahit, Linn.

\begin{tabular}{lllll|l} 
b. bifidu, (Boenn.) ... & $\ldots$ & $\ldots$ & 23
\end{tabular}

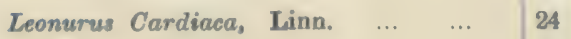




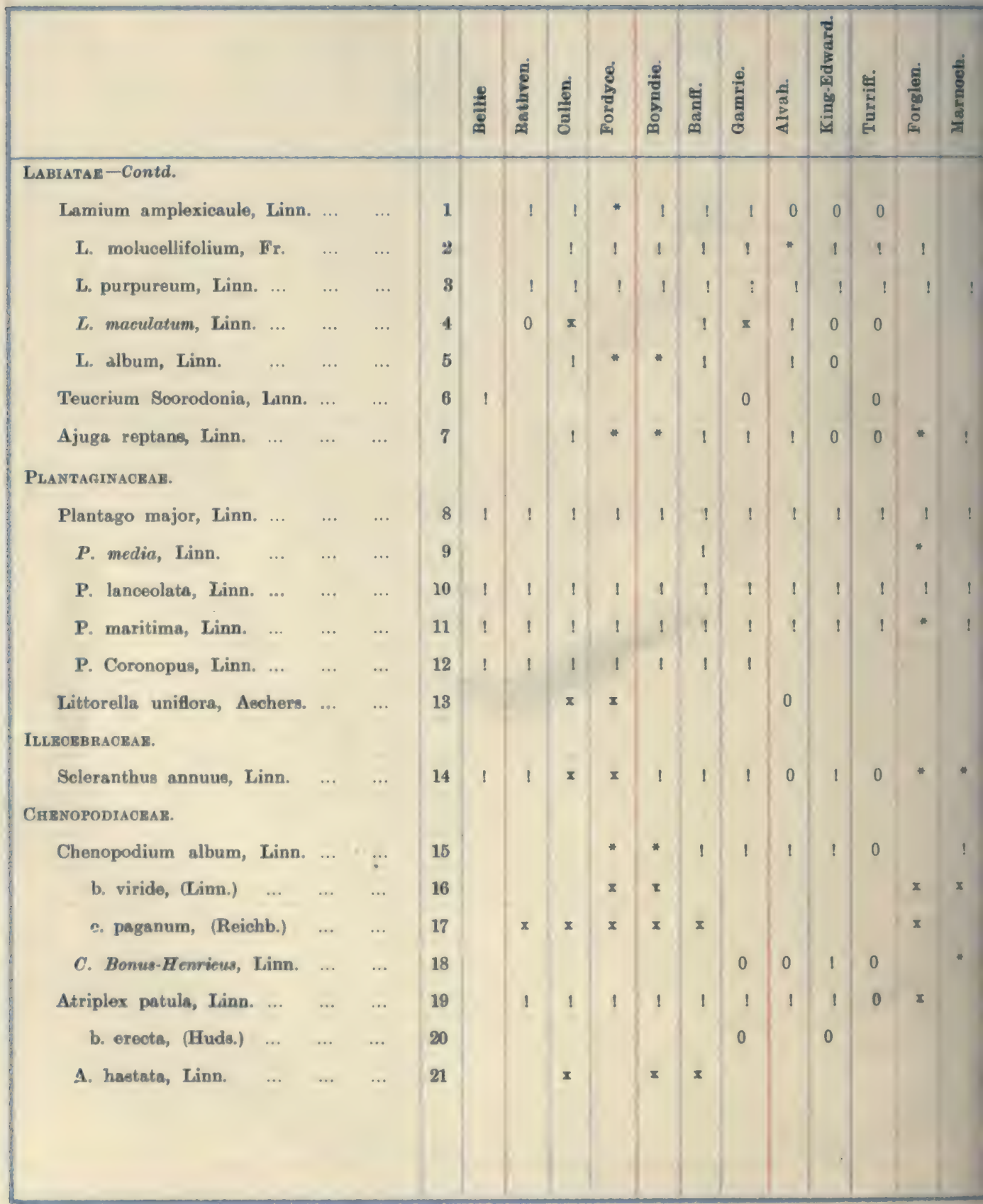




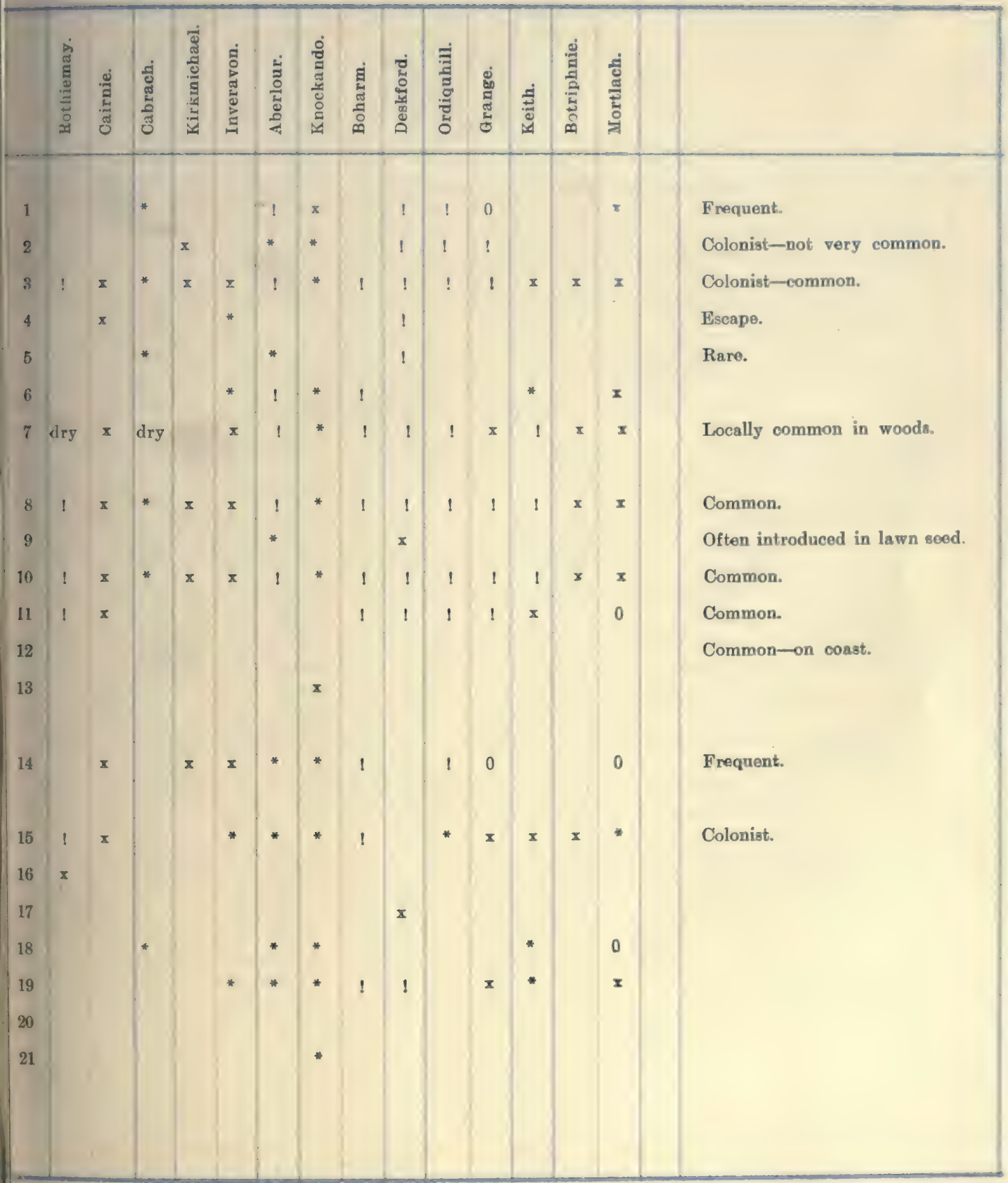




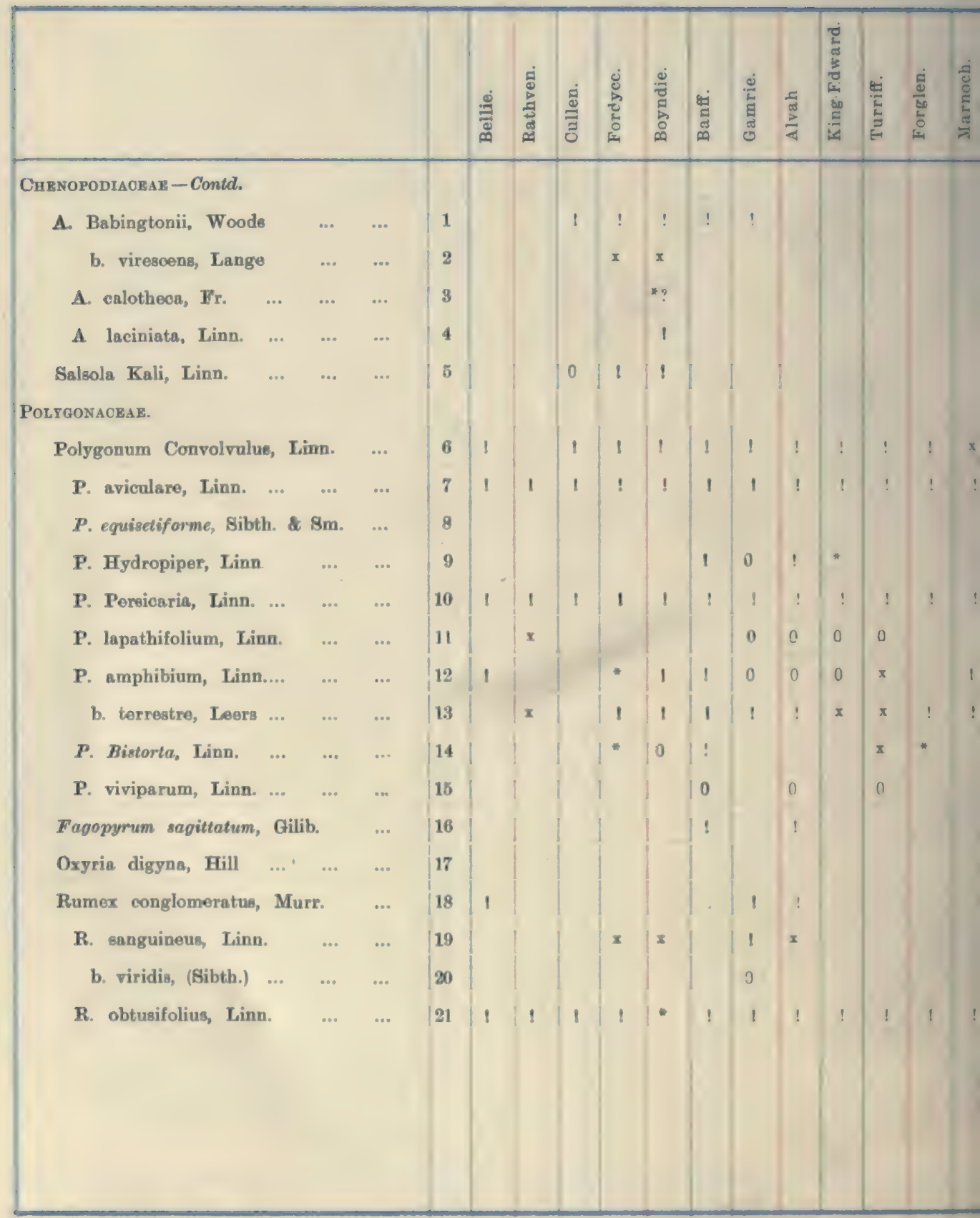




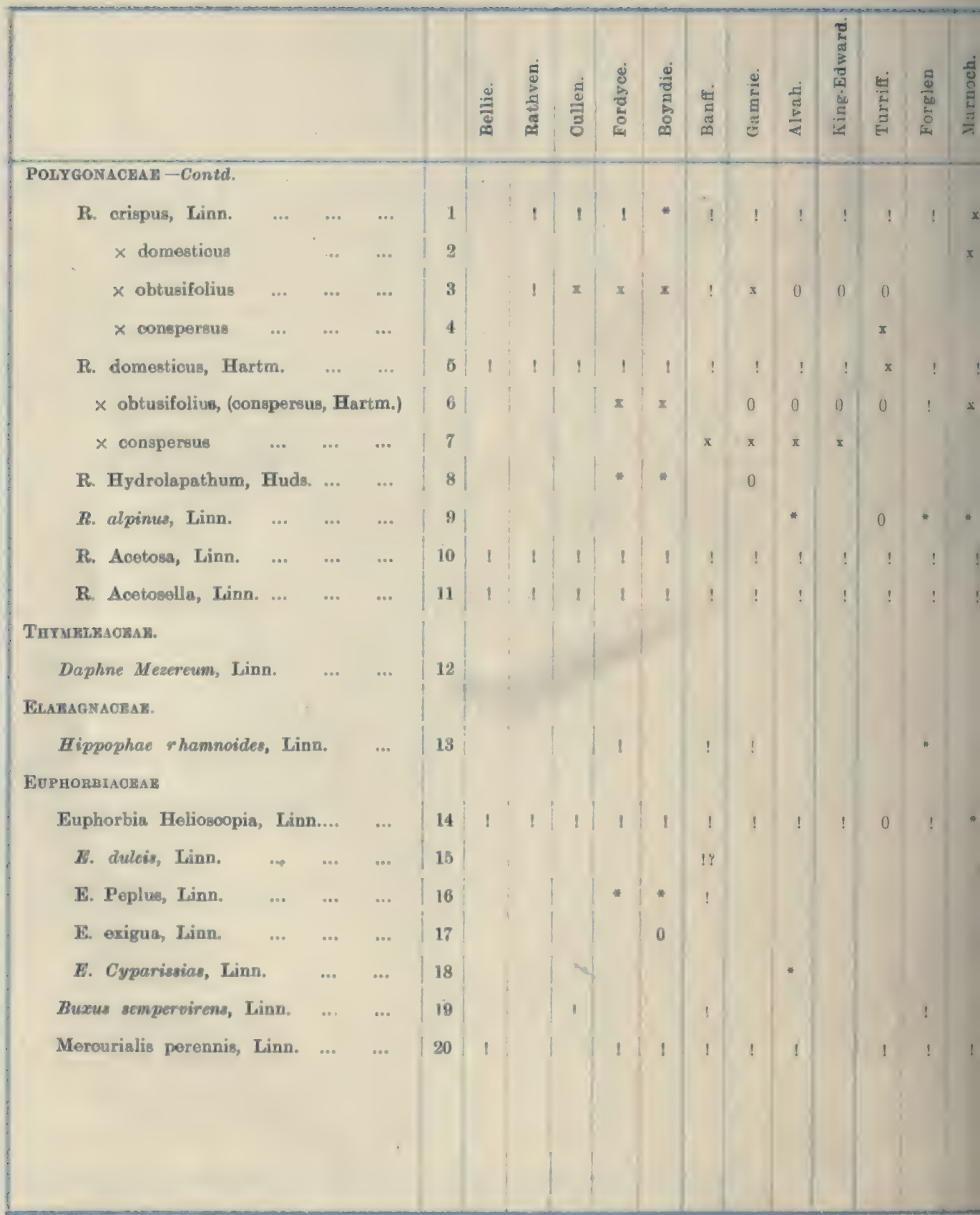




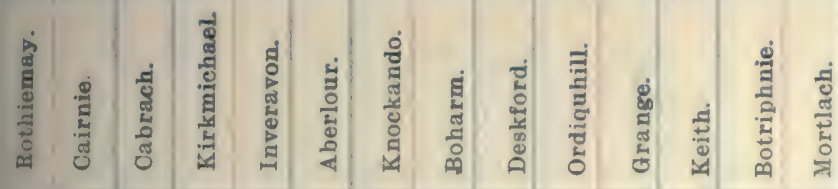

Relio of cultivation?

Common.

Common.

Colonist, in fields and gardens.

Seen once in Duff House Policies

Colonist, in gardens.

Planted.

0 ! 5 x Iocally common. 


$\|1\|\|\|\|\|\|\| \|$

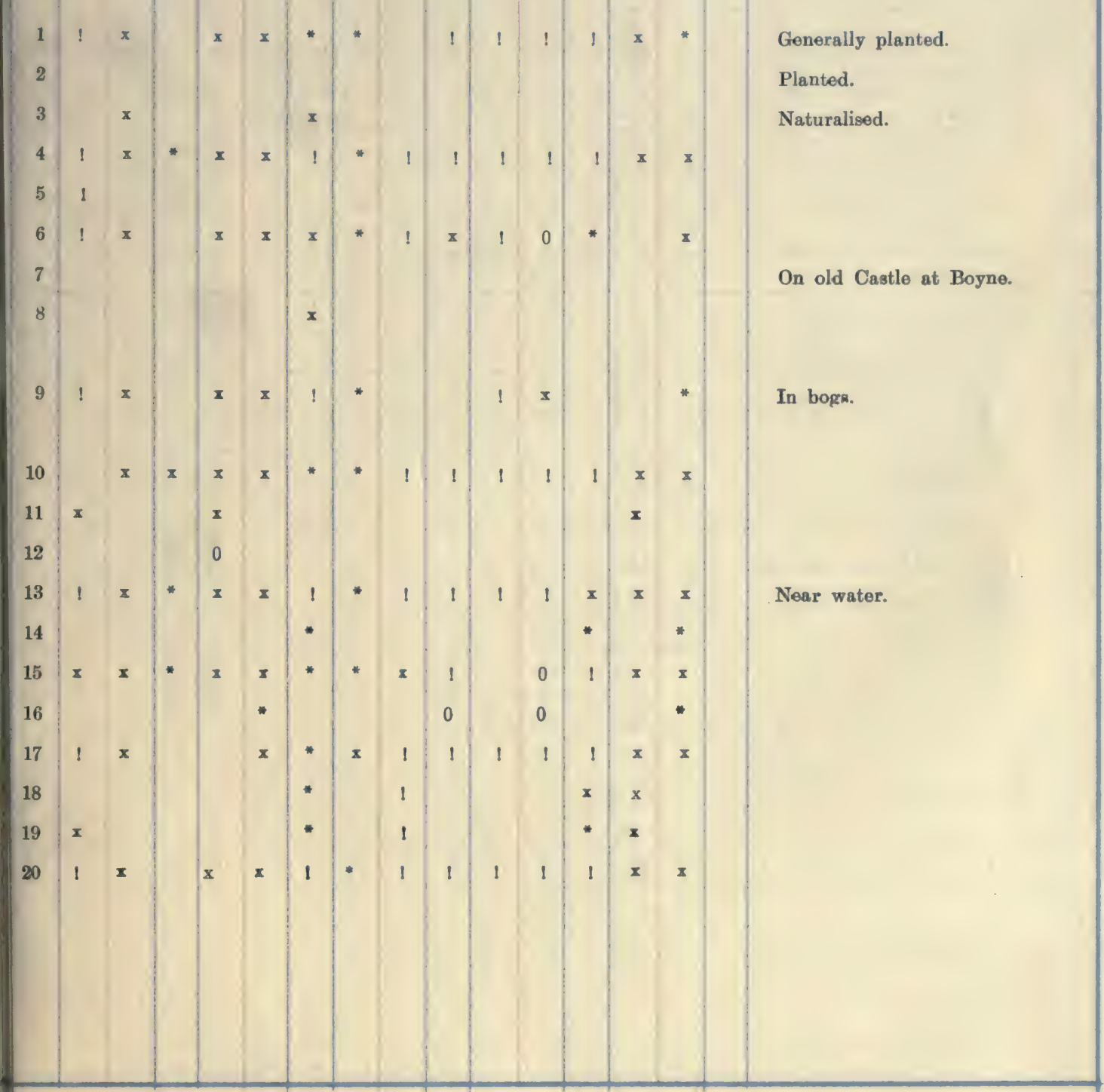




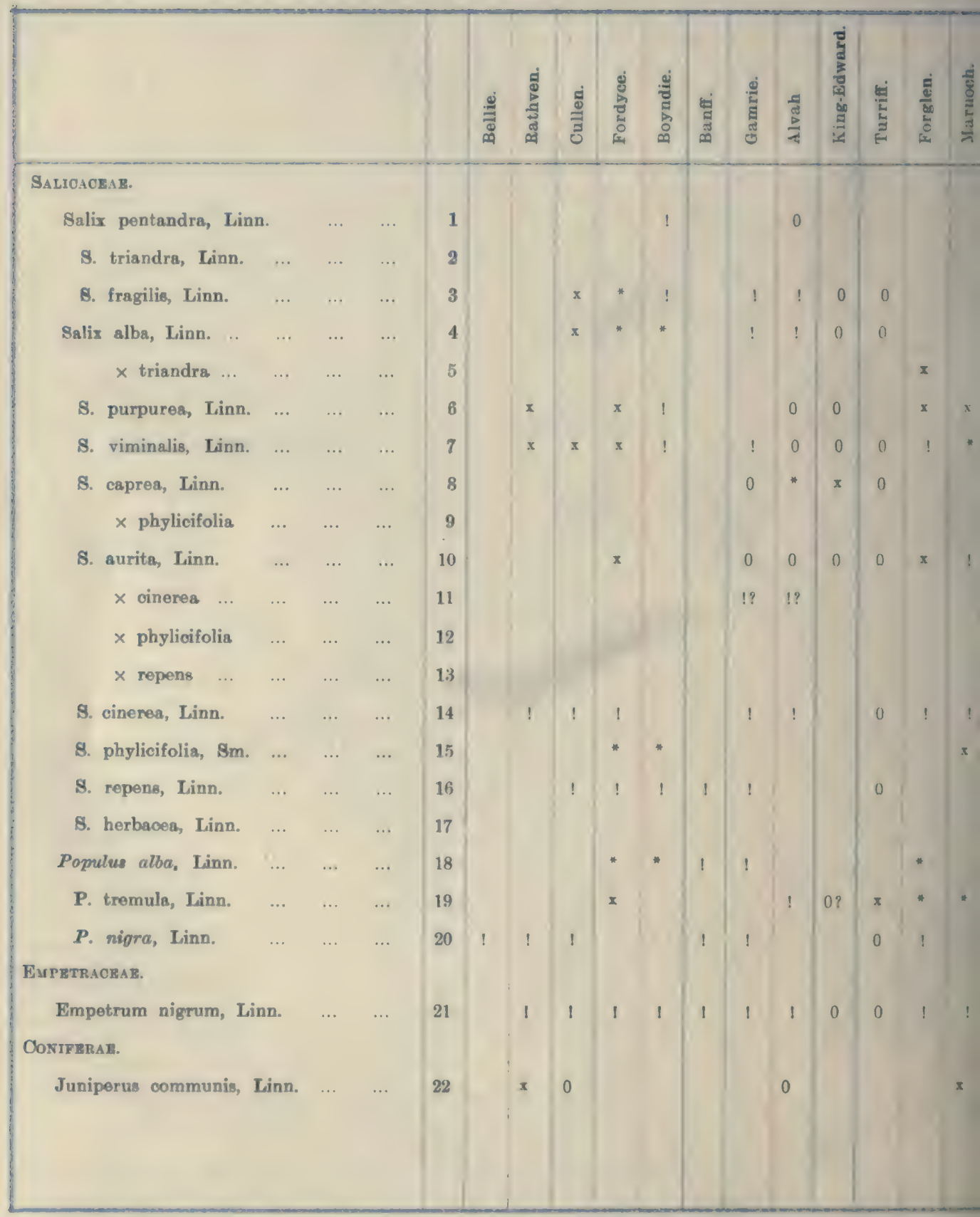




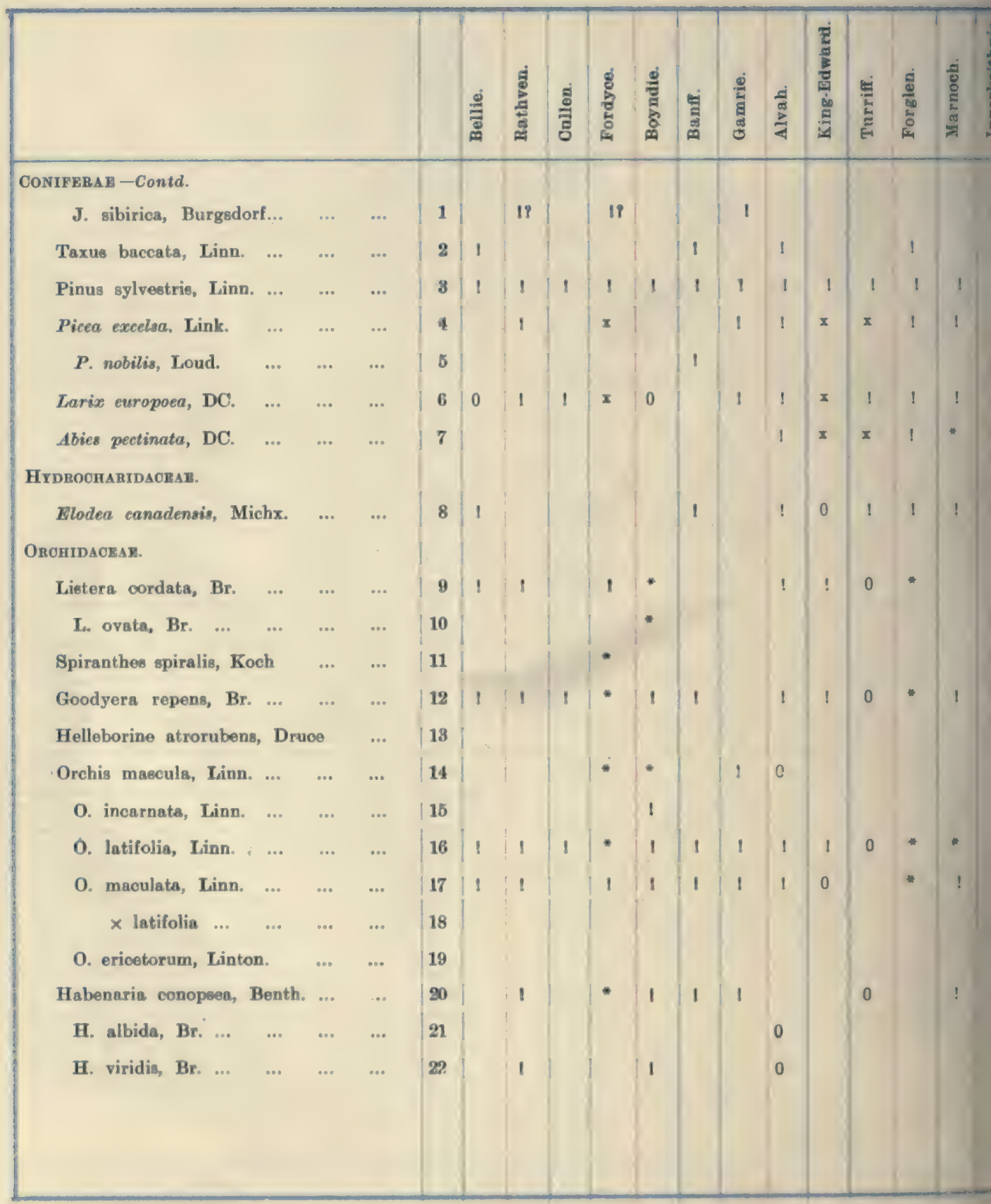




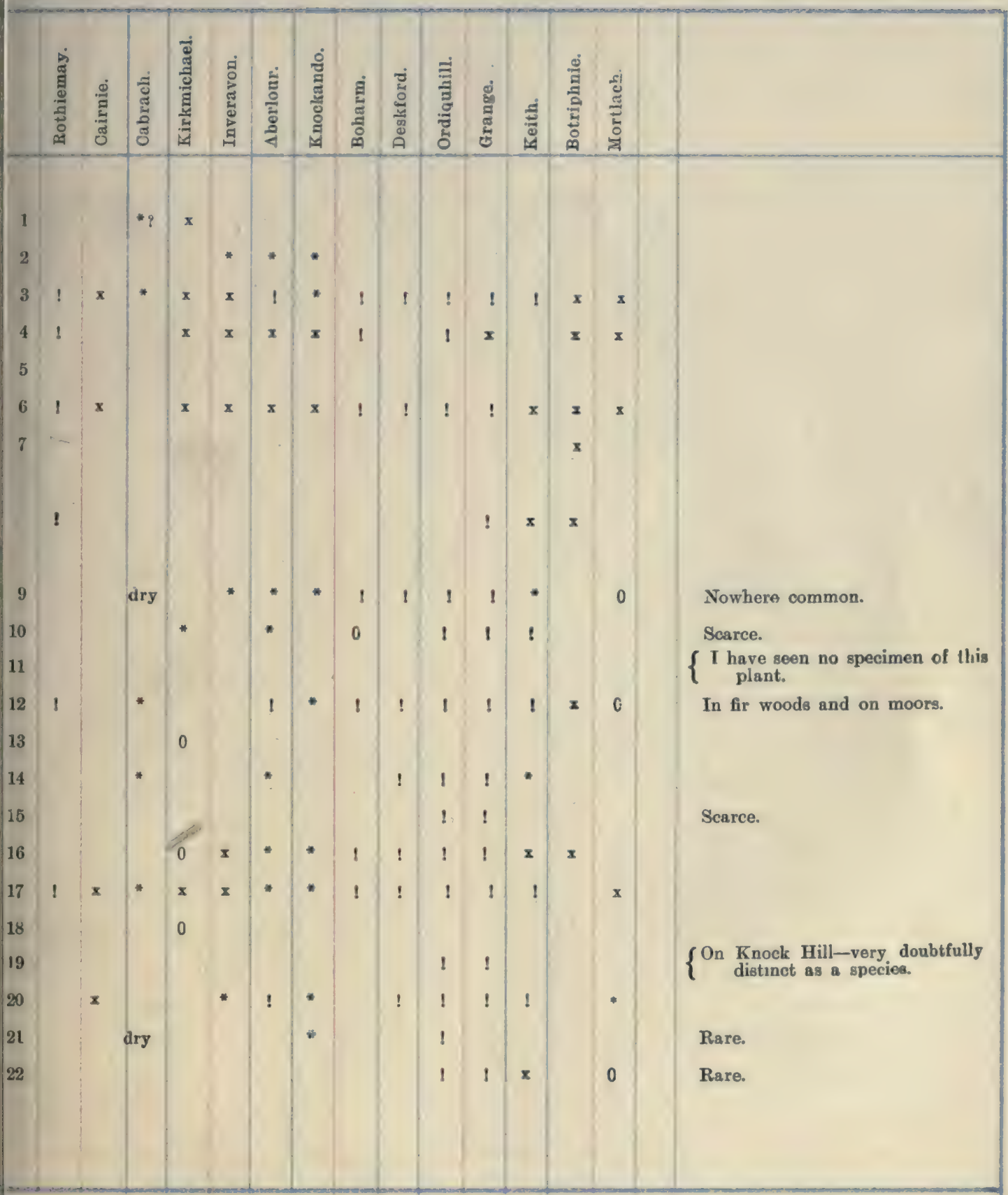




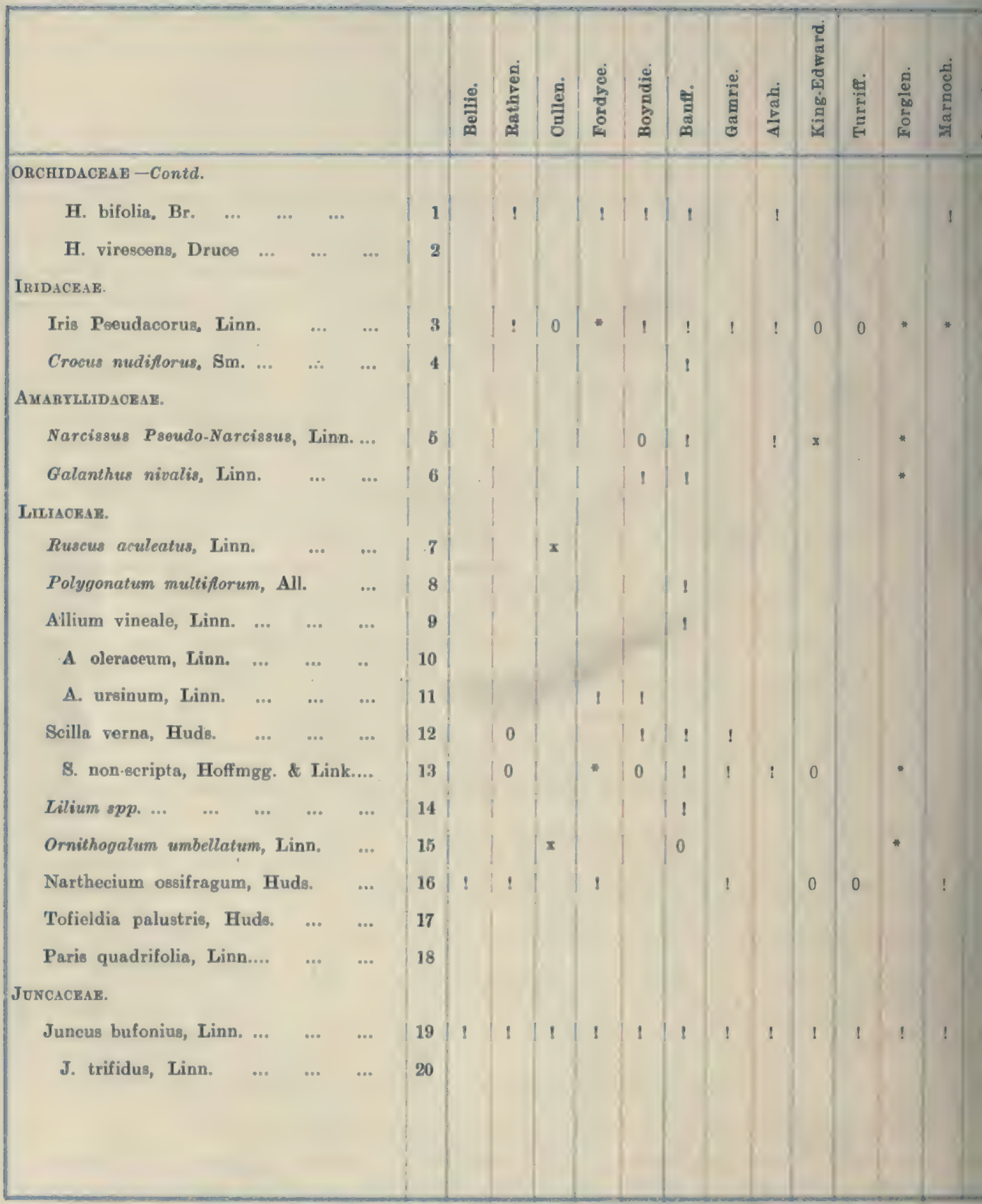




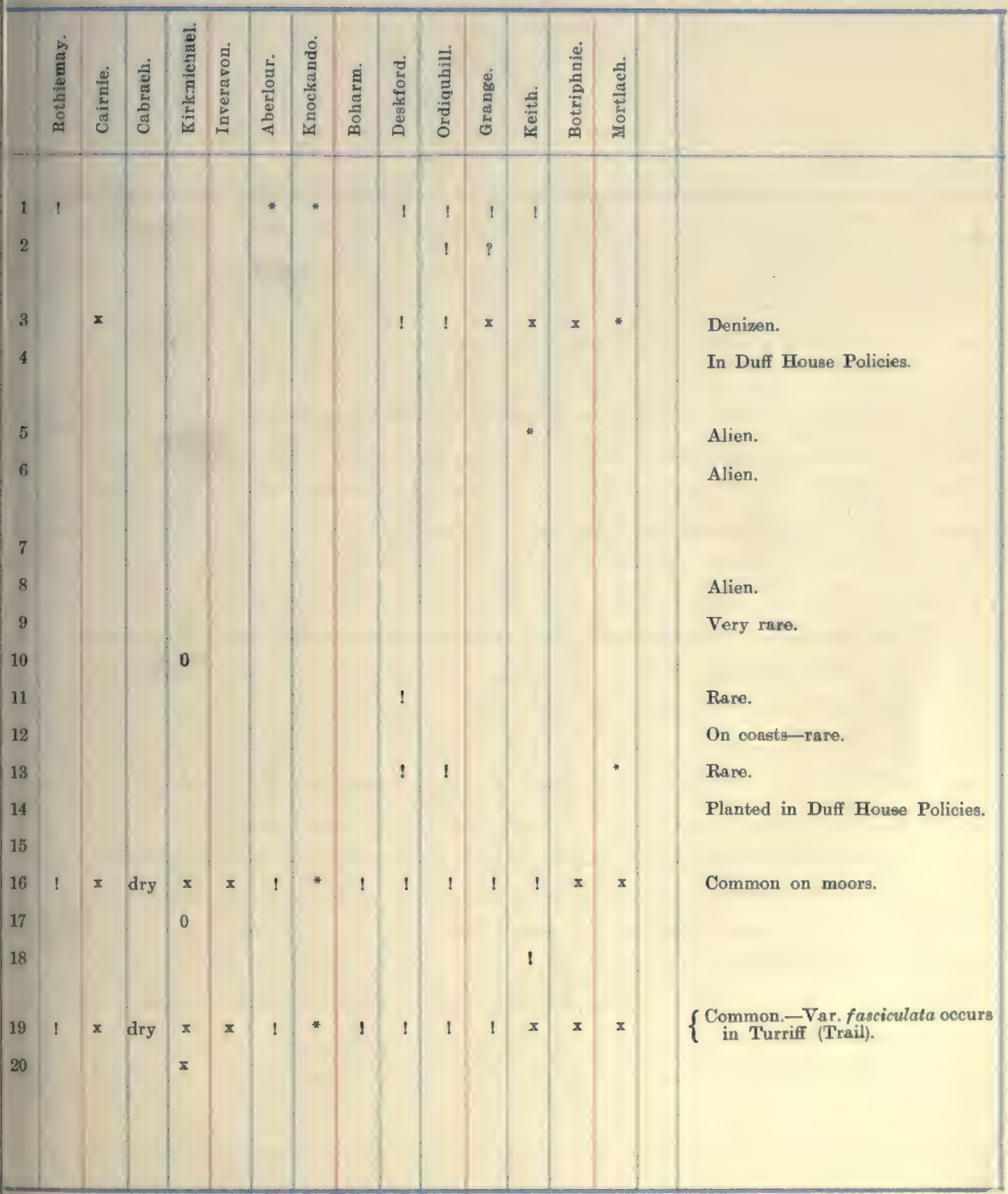




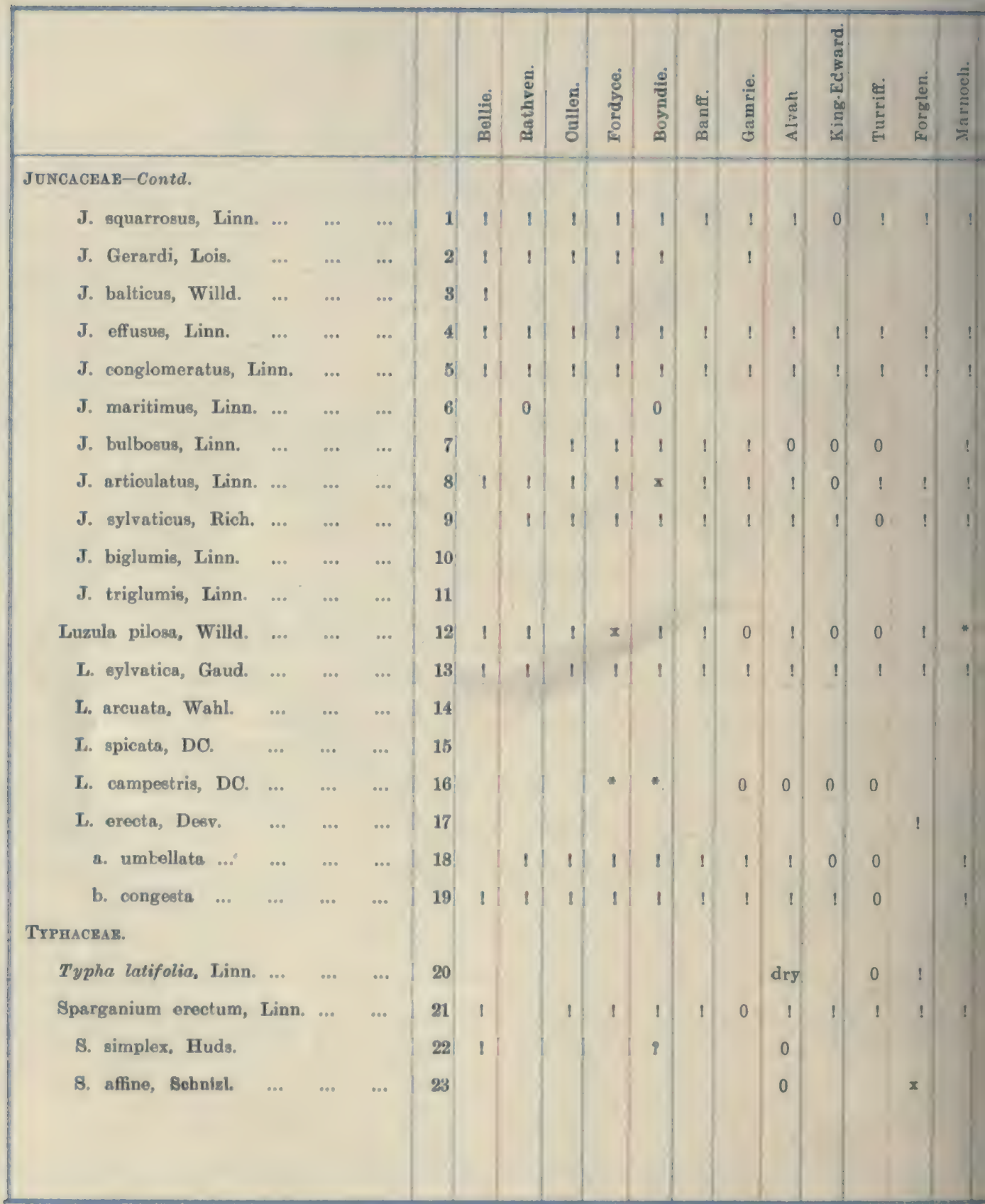




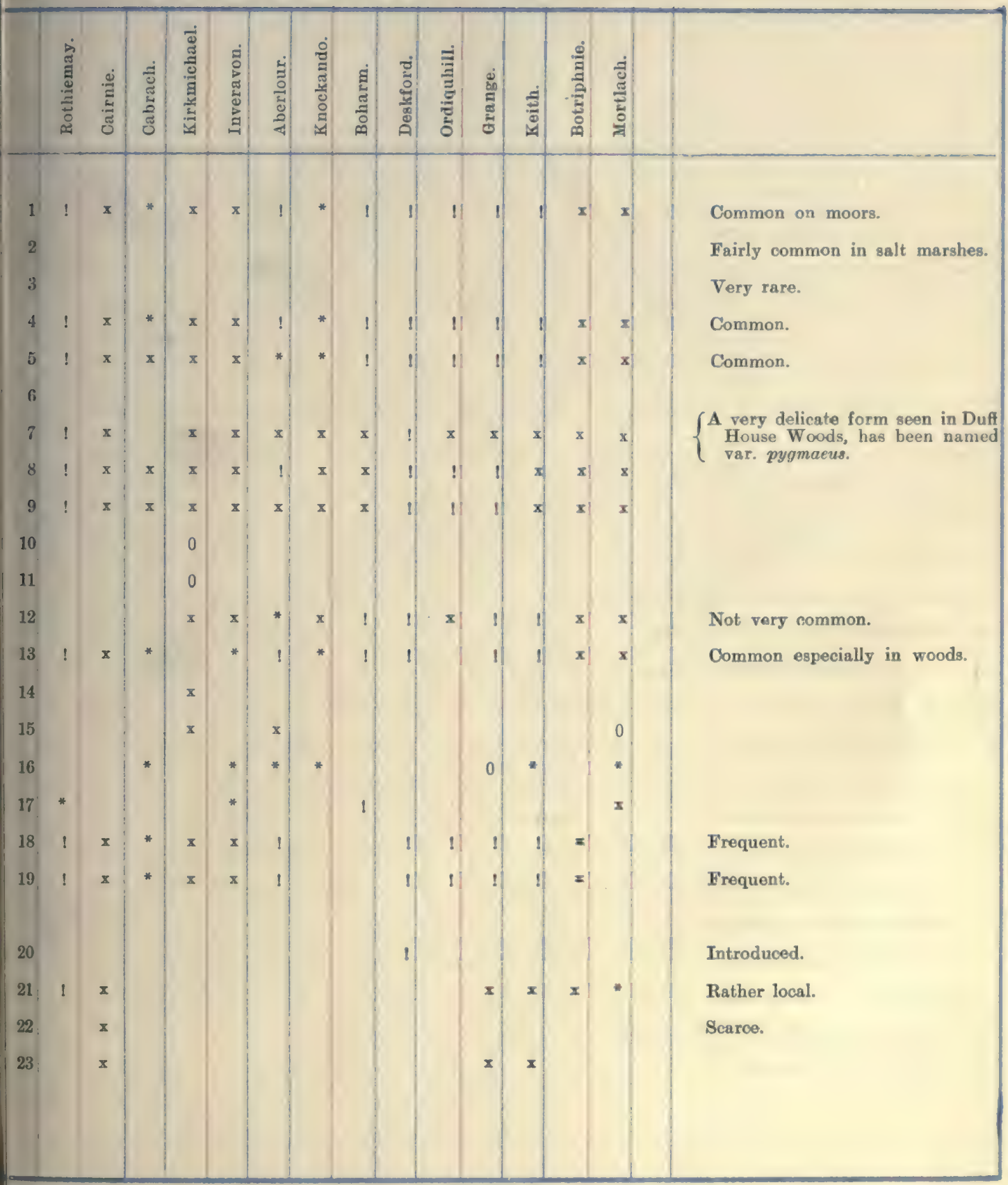




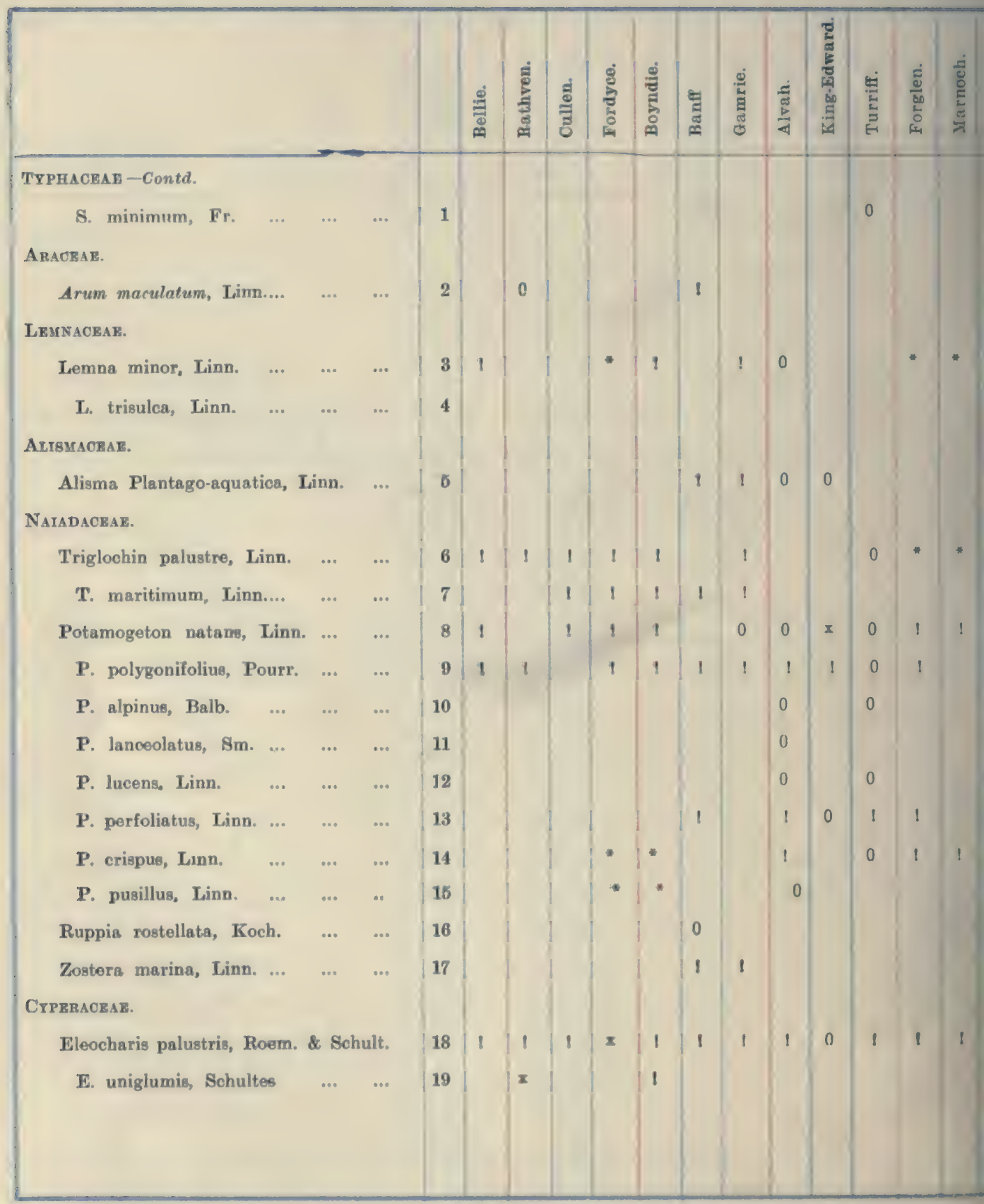




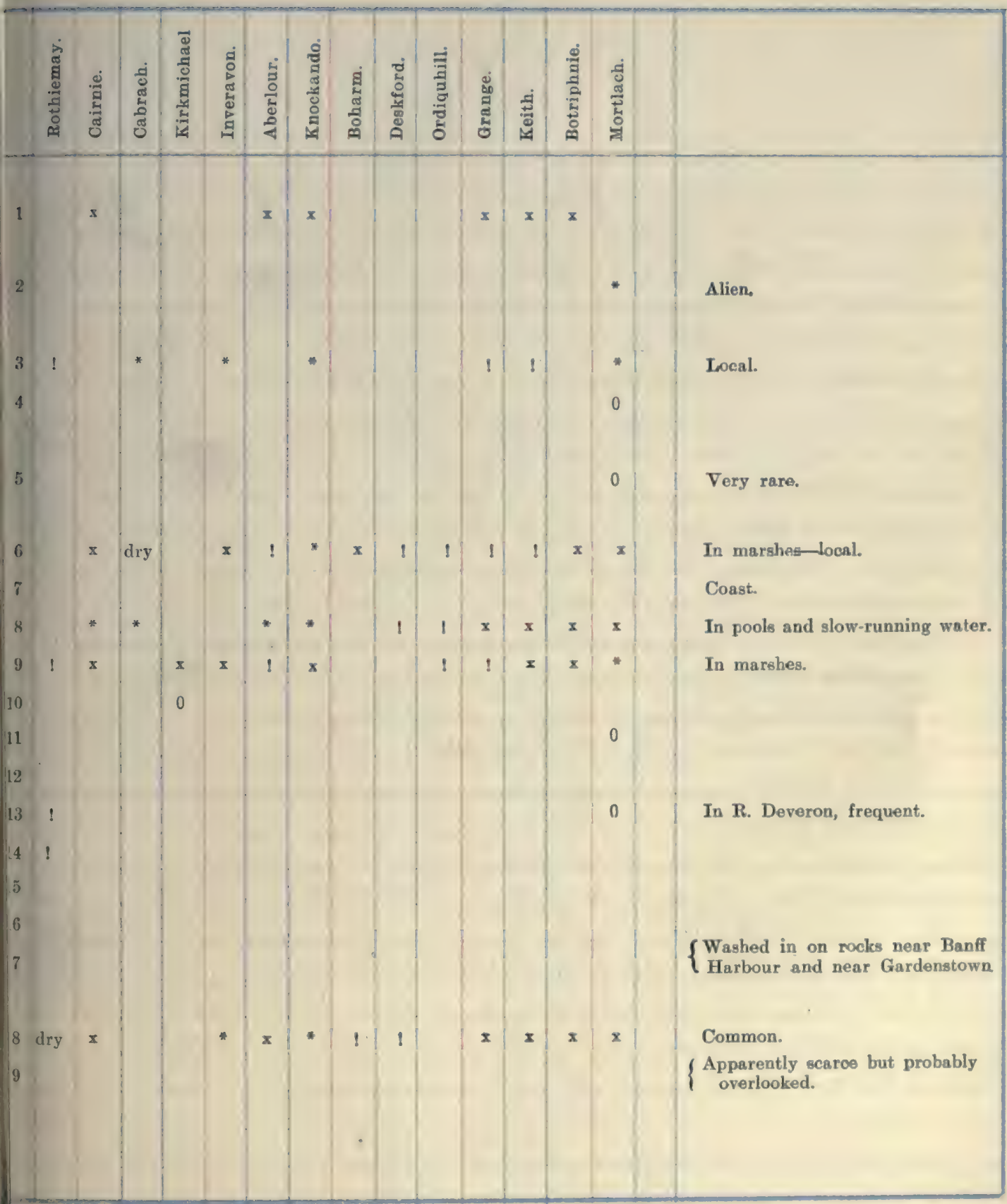




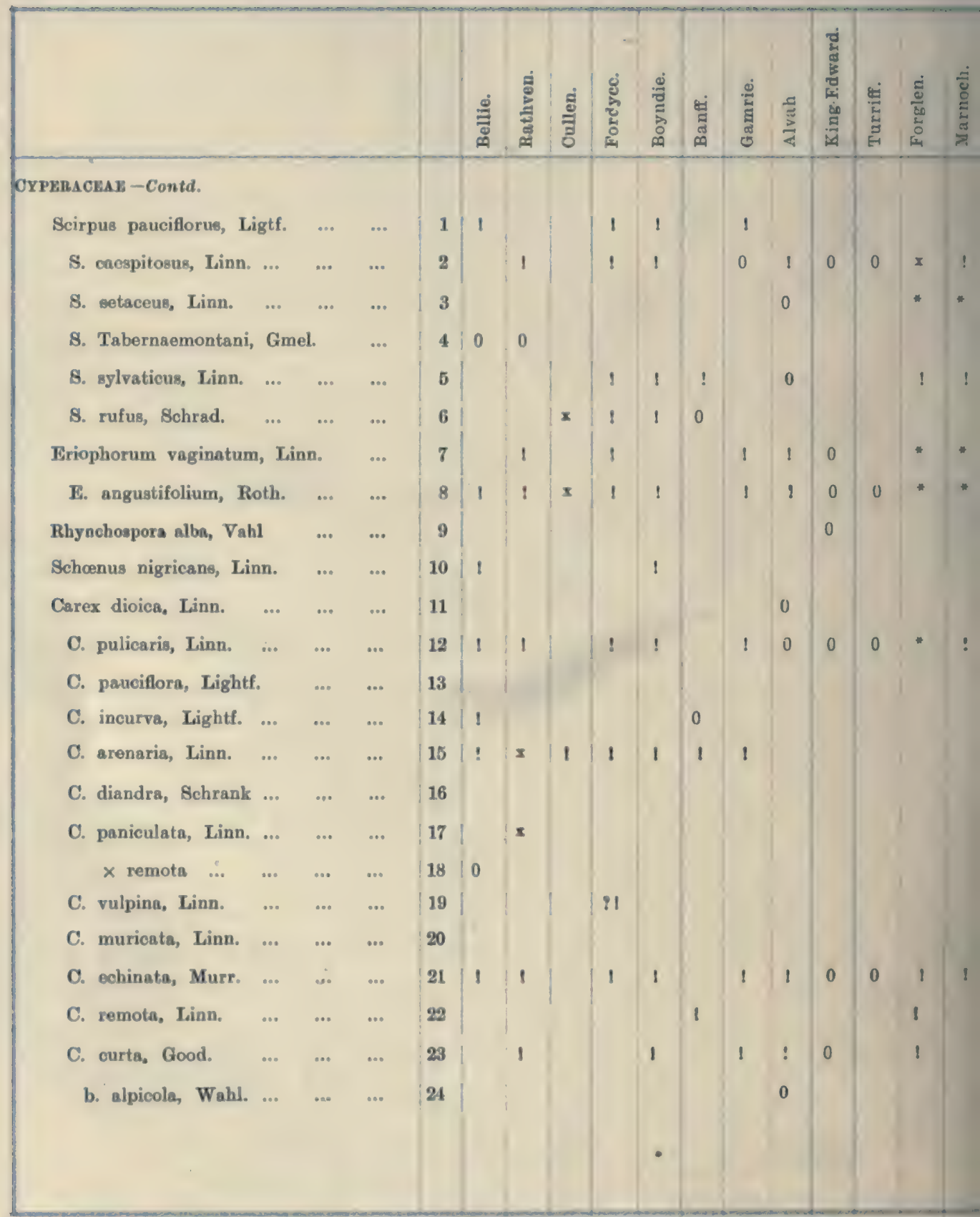




\|\|\|\|\|\|$|\||$

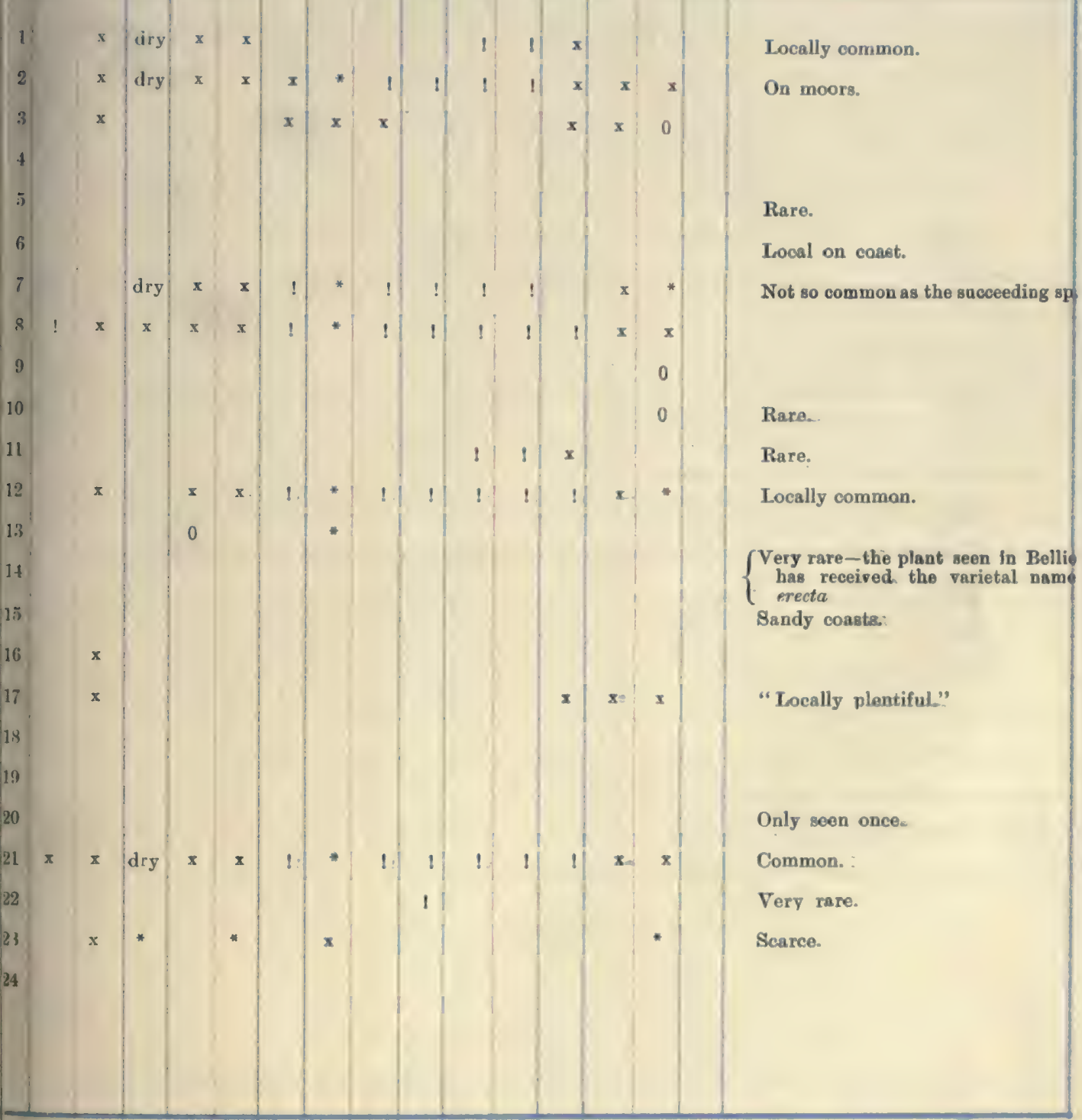


98

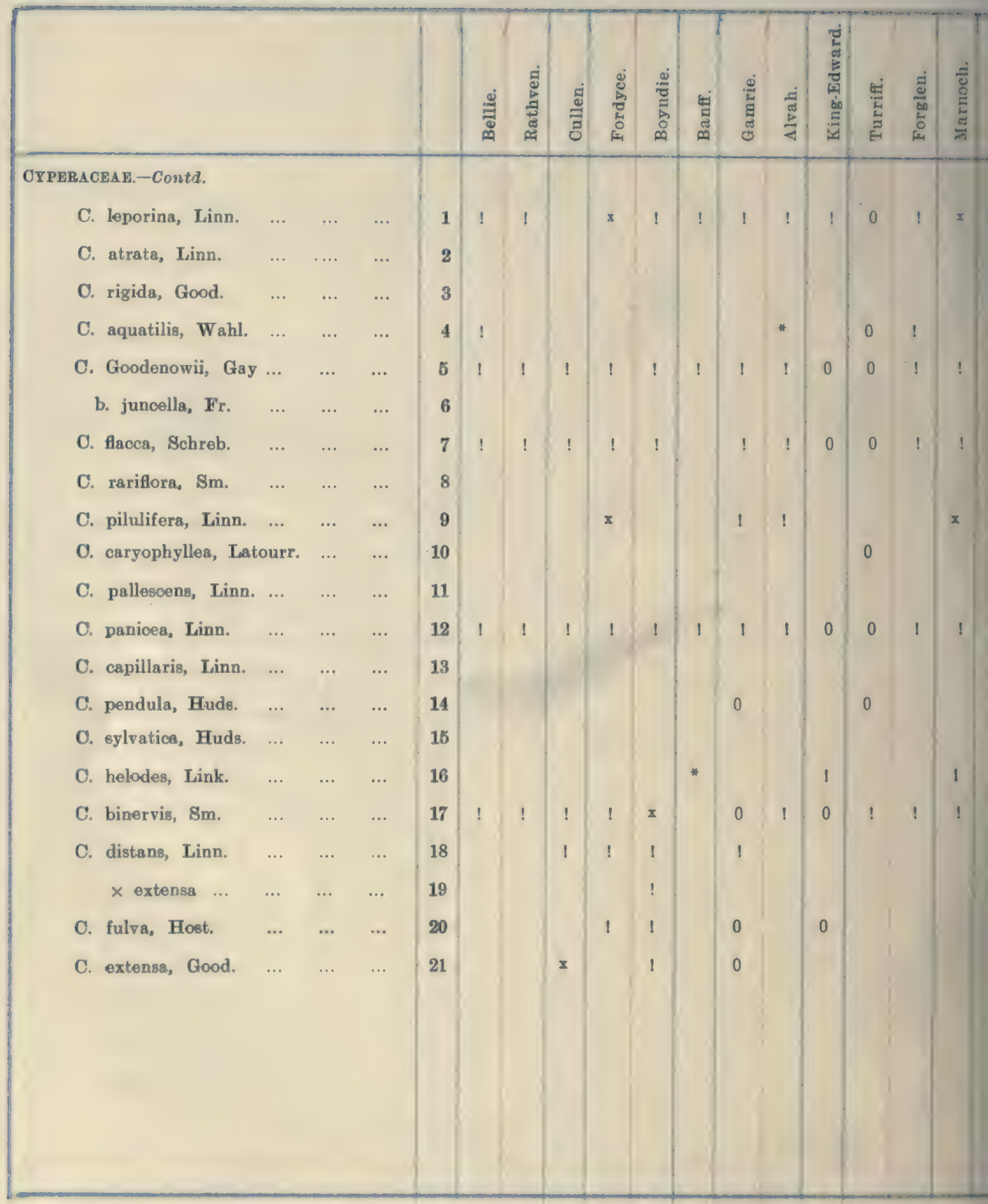




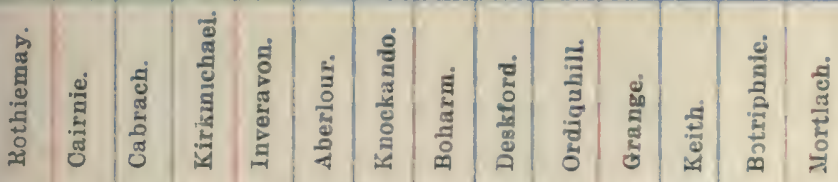

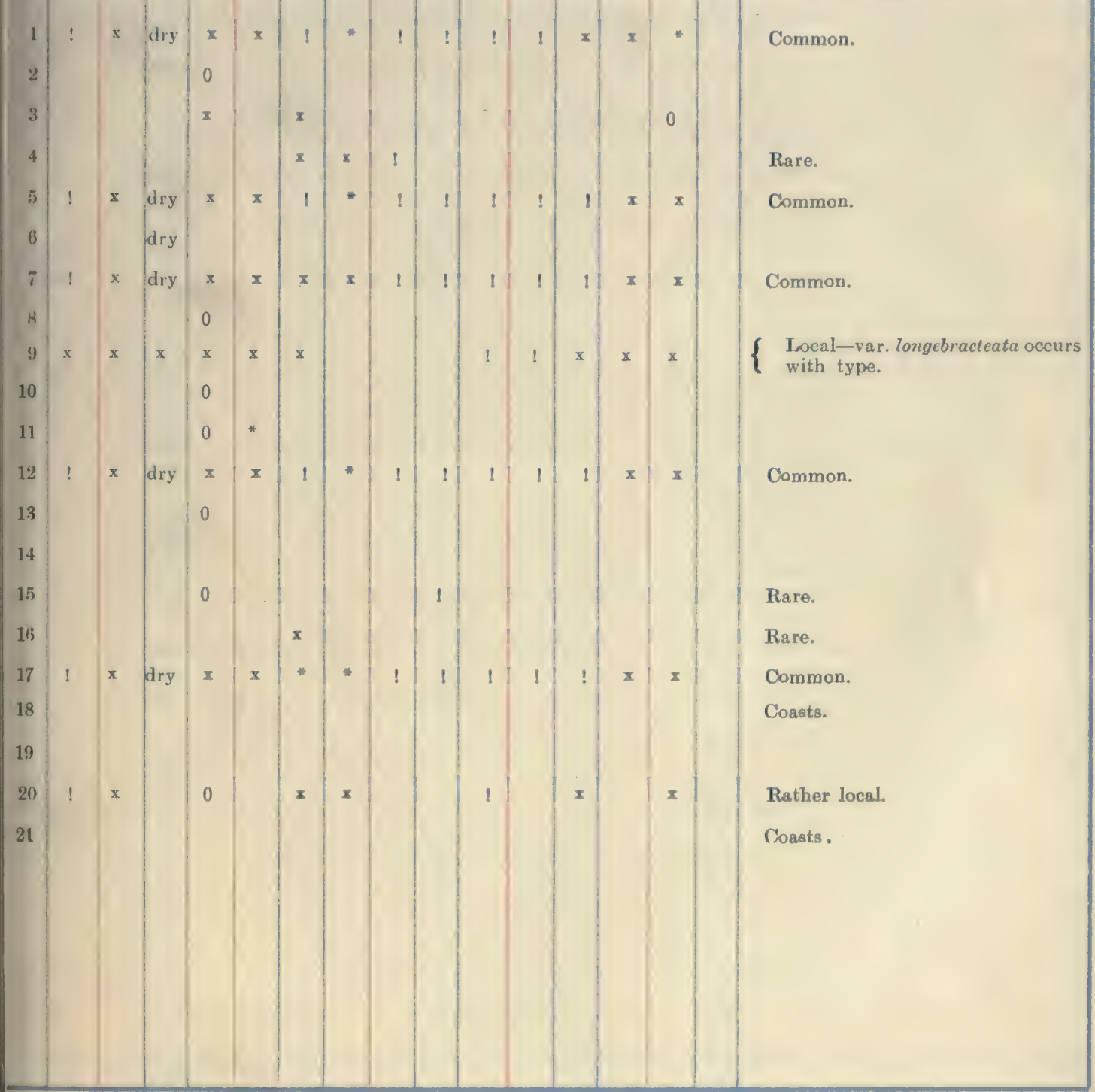




\section{0}

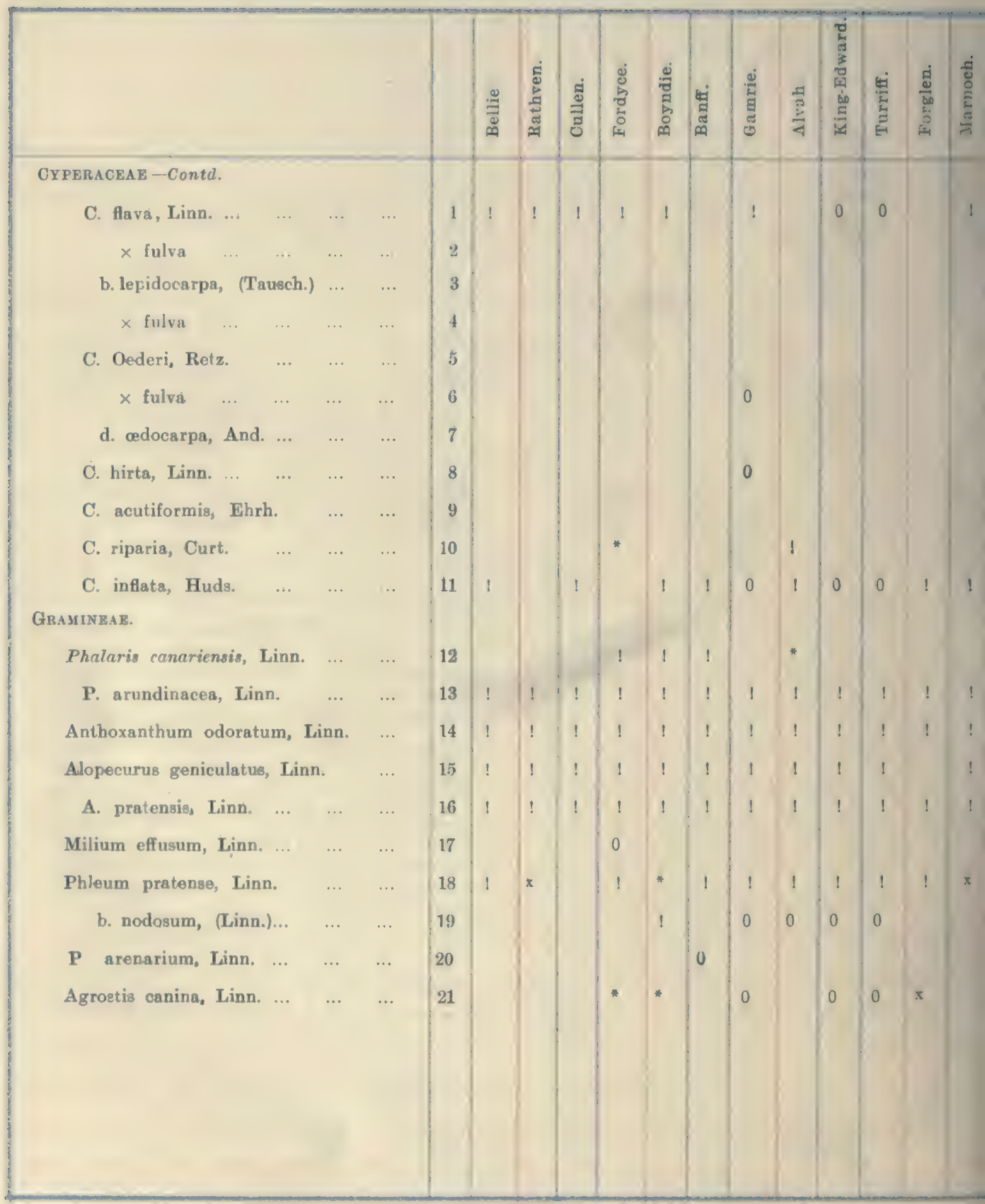




\begin{tabular}{|c|c|c|c|c|c|c|c|c|c|c|c|c|c|}
\hline 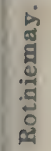 & 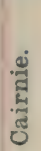 & 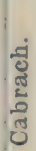 & $\frac{\partial}{3}$ & 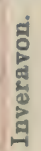 & 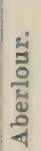 & 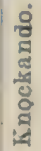 & 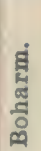 & 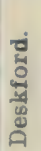 & 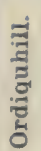 & 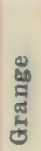 & 预 & 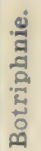 & है \\
\hline
\end{tabular}

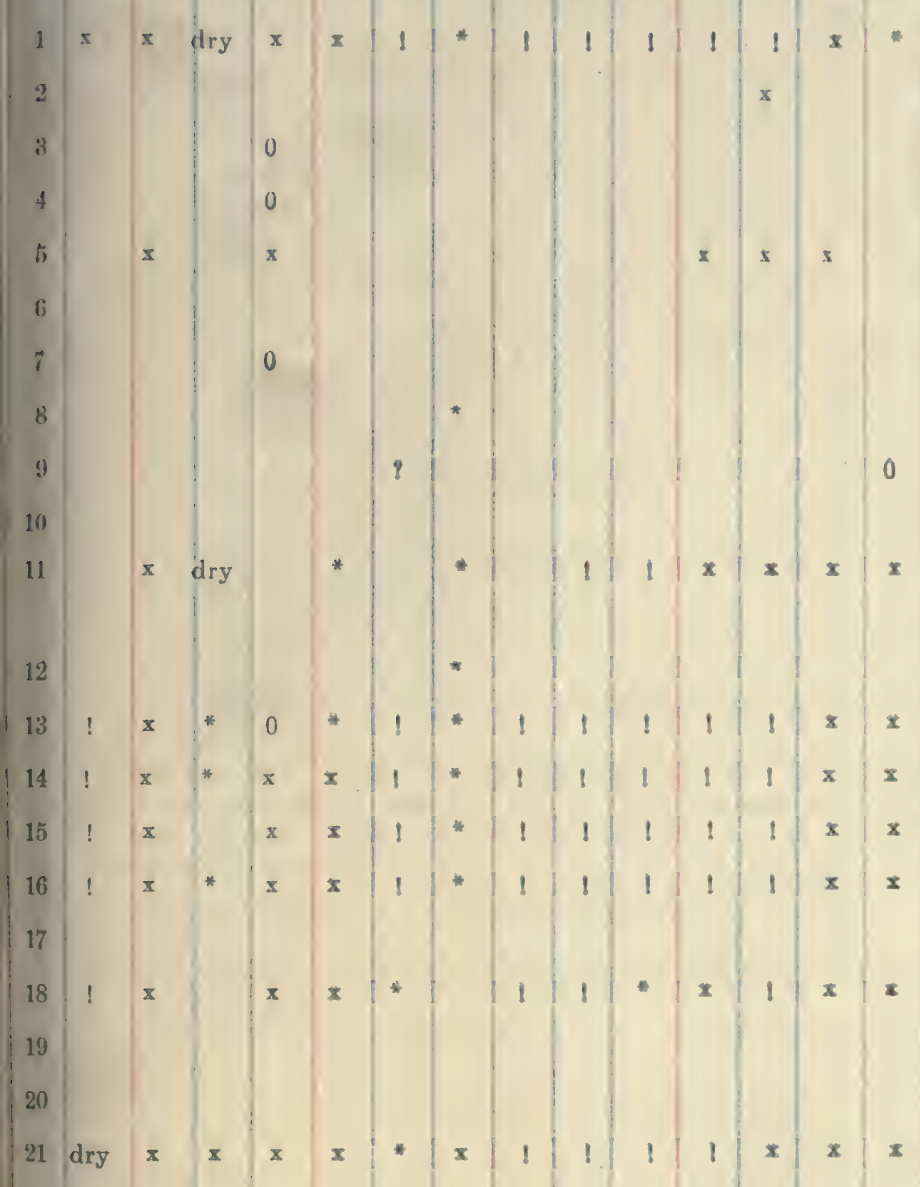

Common.

Rare.

Locally common.

Casurl.

Common by streams.

Common.

Common.

Common.

Frequent. 


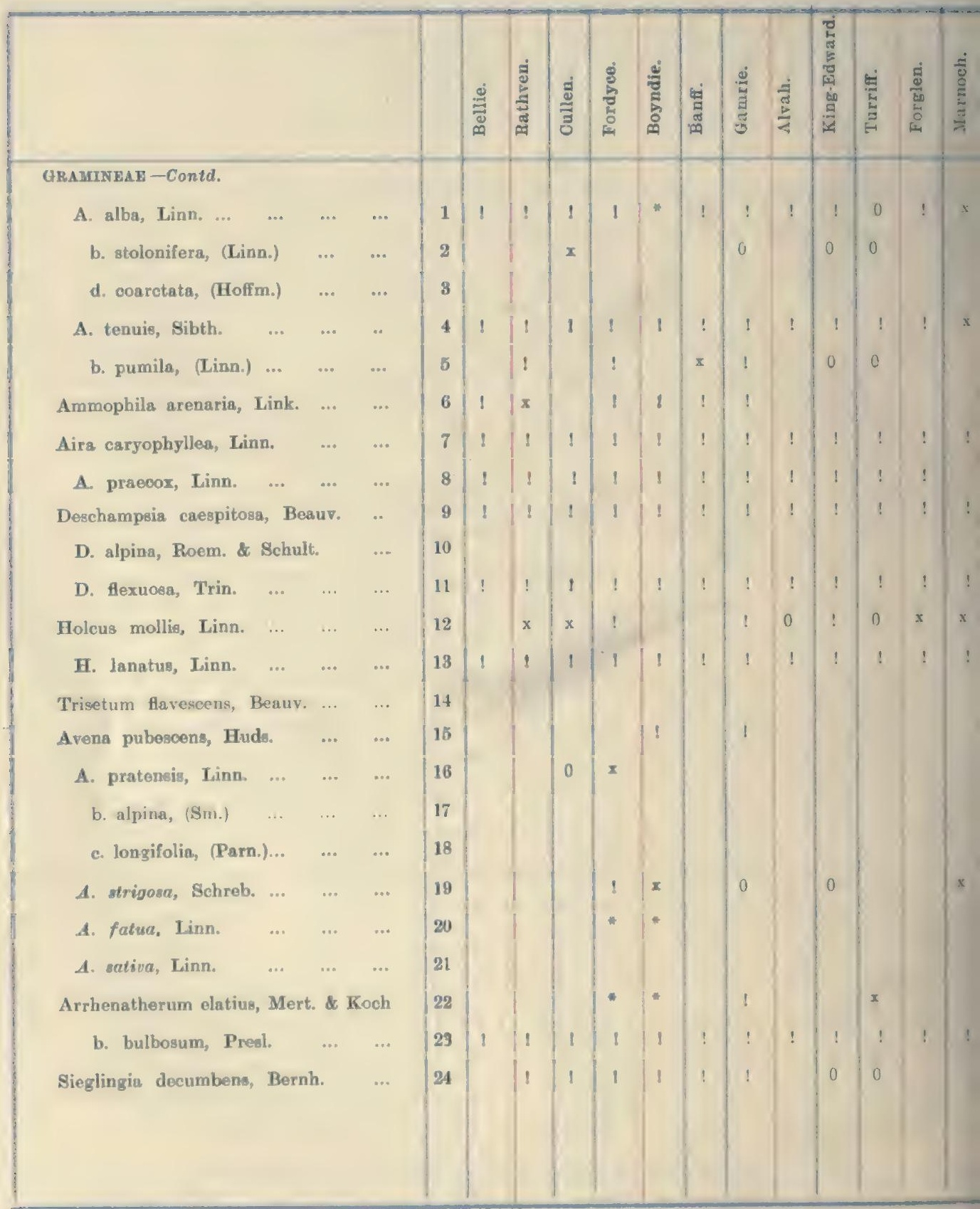




IIIIIIIIIIII

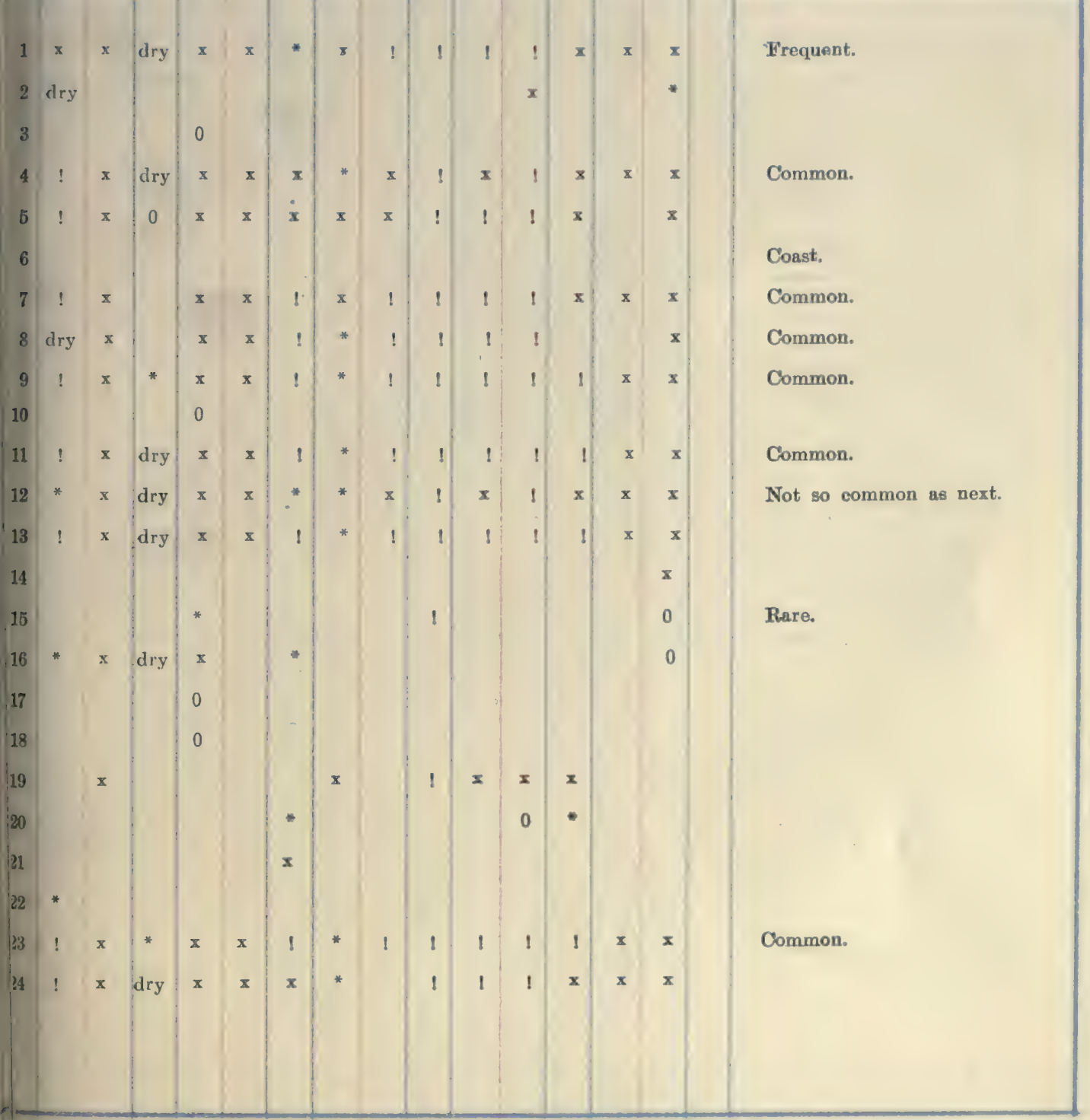




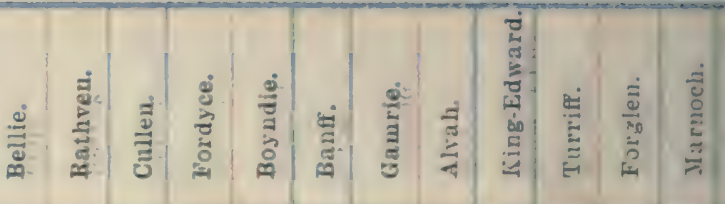

GRAMINEAE - Contil.

\begin{tabular}{ll|l|} 
Phragmites osmmunis, Trin.... & ... & 1
\end{tabular}

Cynosurus cristatus, Linn.

Koeleria gracilis, Pers. ...

๑. britannica, Domin

Molinia corulea, Moench.

b. depauperata, $\left(\begin{array}{lll|l}\text { Lindl. }) & \ldots & \ldots & 6\end{array}\right.$

\begin{tabular}{ll|l|l|l|l} 
Catabrosa aquatica, Beauv: & ... & $\ldots$ & 7
\end{tabular}

Melica nutans, Linn: ... ... ...

Dactylis glomerata, Lims:

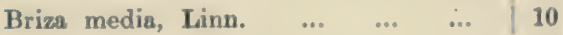

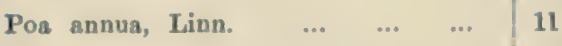

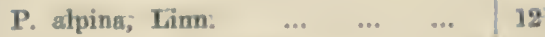

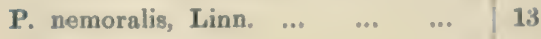

\begin{tabular}{ll|l|l} 
d. divaricata, Syme ... &.. & 14
\end{tabular}

\begin{tabular}{llll|l} 
P. comprossa, Linn. ... & $\ldots$ & $\ldots$ & 13
\end{tabular}

P. pratensis, Linn. ... $\ldots . \quad \ldots \quad|16|$

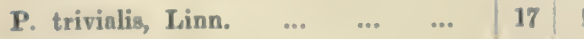

\begin{tabular}{llll|l} 
Glyceria fluitans, Br. & .. & $\ldots$ & $\ldots$ & 18
\end{tabular}

\begin{tabular}{lllll|l} 
G. plicata, Fr. .. & $\ldots$ & $\ldots$ & $\ldots$ & 19
\end{tabular}

\begin{tabular}{llll|l} 
G. aquatica, Wahlb. .. & $\ldots$ & $\ldots$ & 20
\end{tabular}

G. maritima, Mert. \& Koch. ... 21

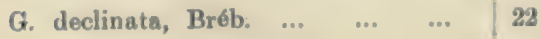

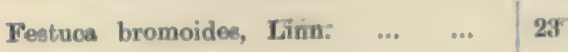

F. ovina, Linn. 


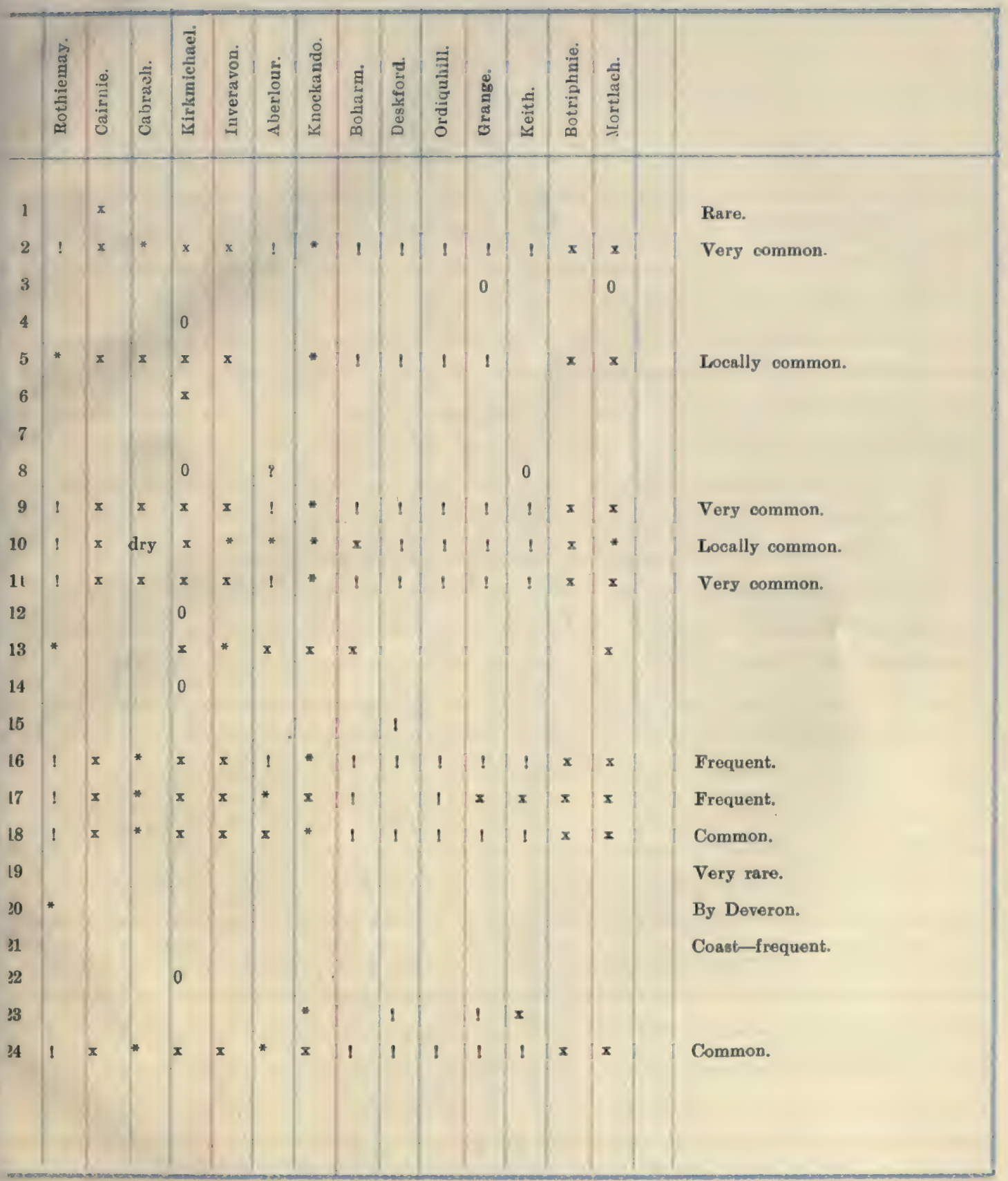




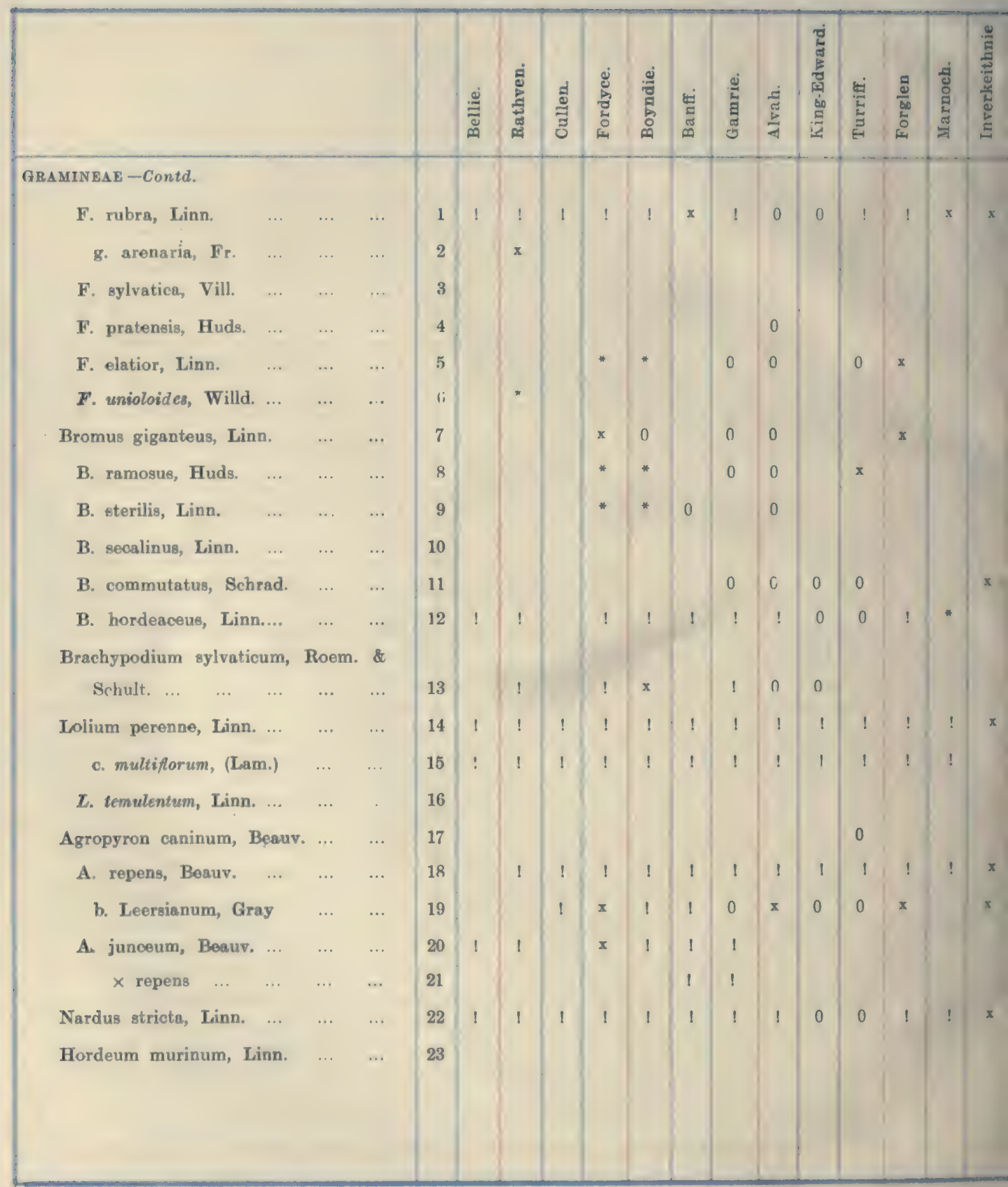




$$
\text { IIIIIIIIIIII }
$$

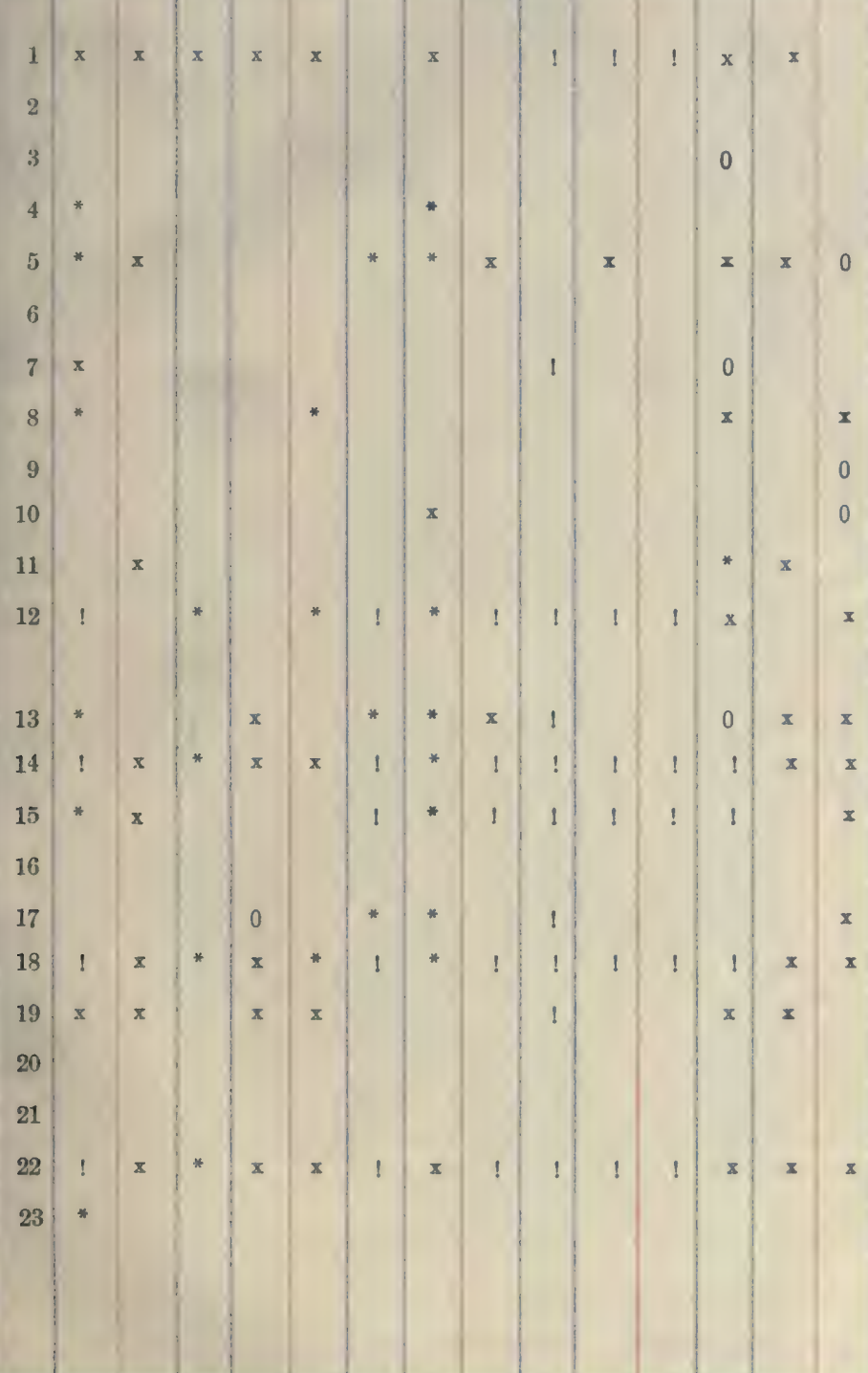

f. vivipera also occurs.

Rare.

Common-m. pumila also occurs.

Local.

Very common.

Common.

Rare.

Very common.

Common.

Coast.

Coast-rare.

"Only one specimen." 


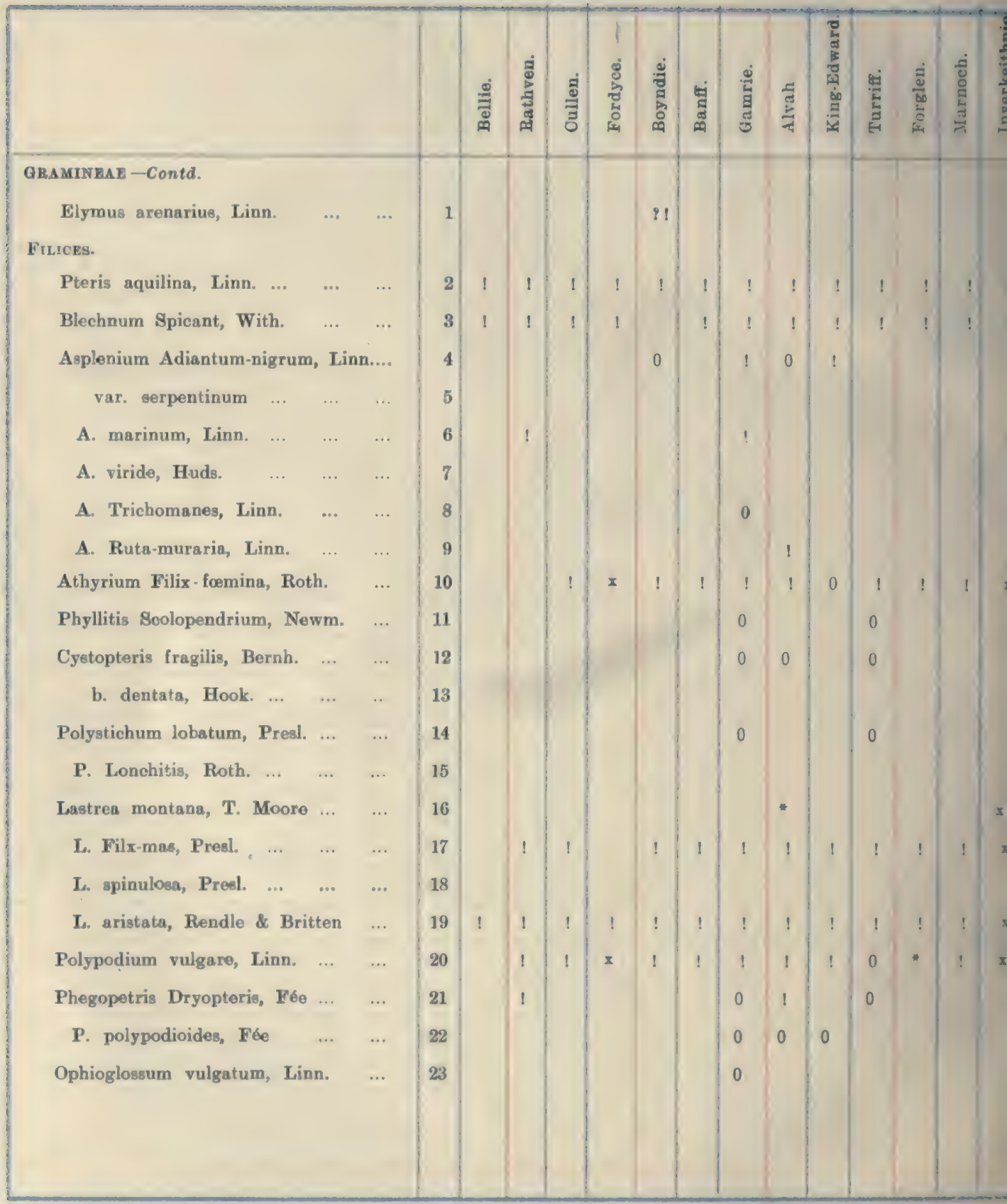




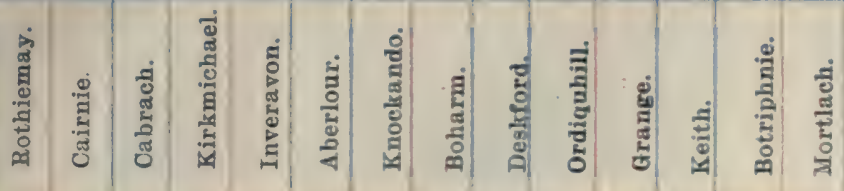

Doubtful-flowers not seen.

Common.

Common.

Rare.

On serpentine rocks.

Coast-local.

Rare.

Common.

Common.

Common.

Common.

Local-nowhere common.

Local-nowhere common. 


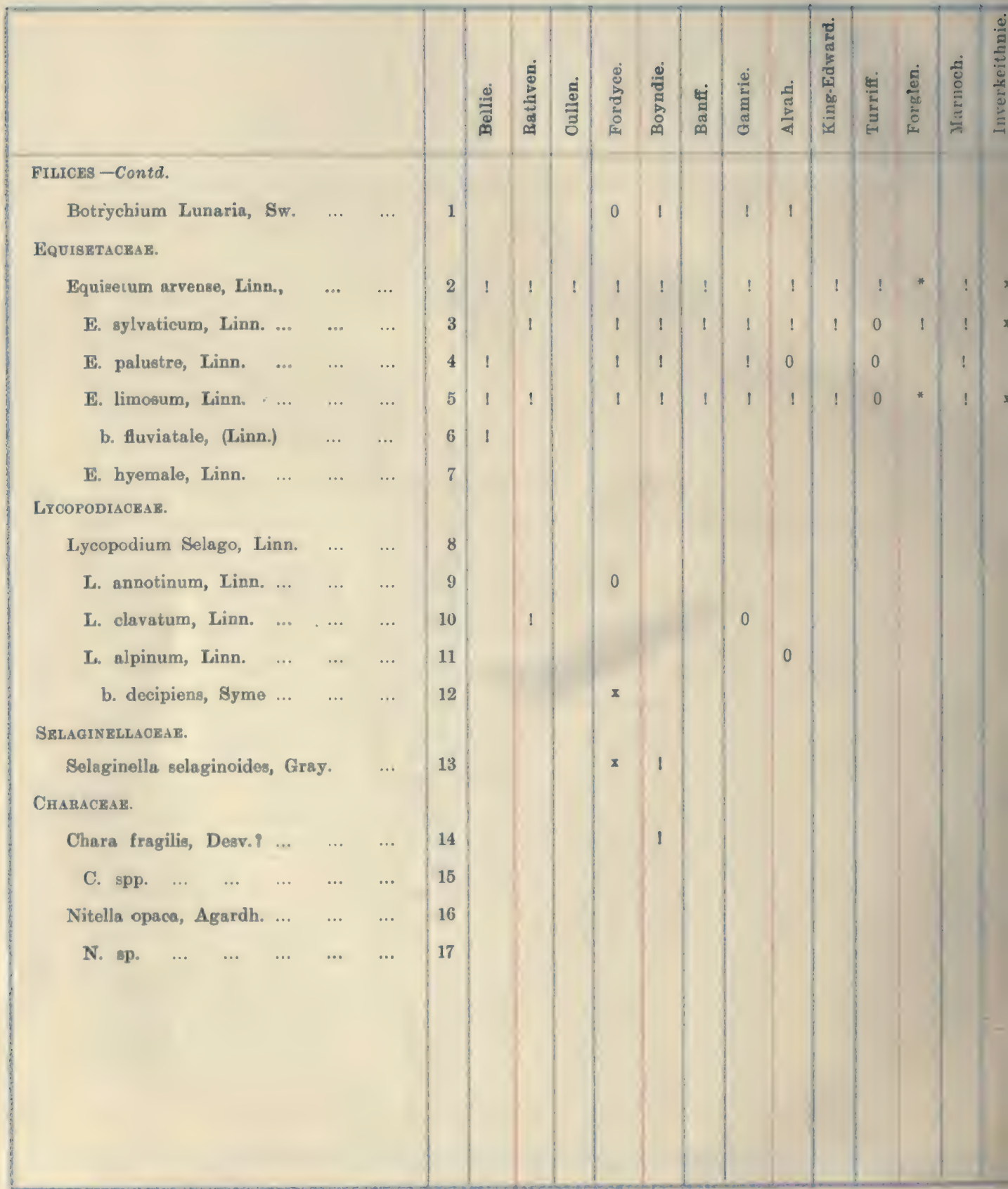




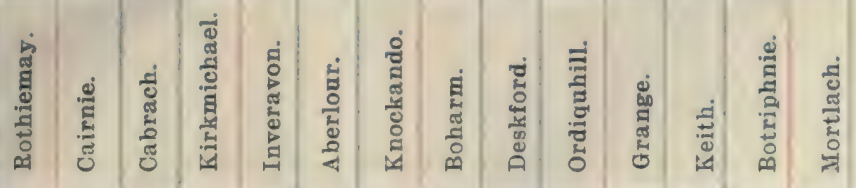

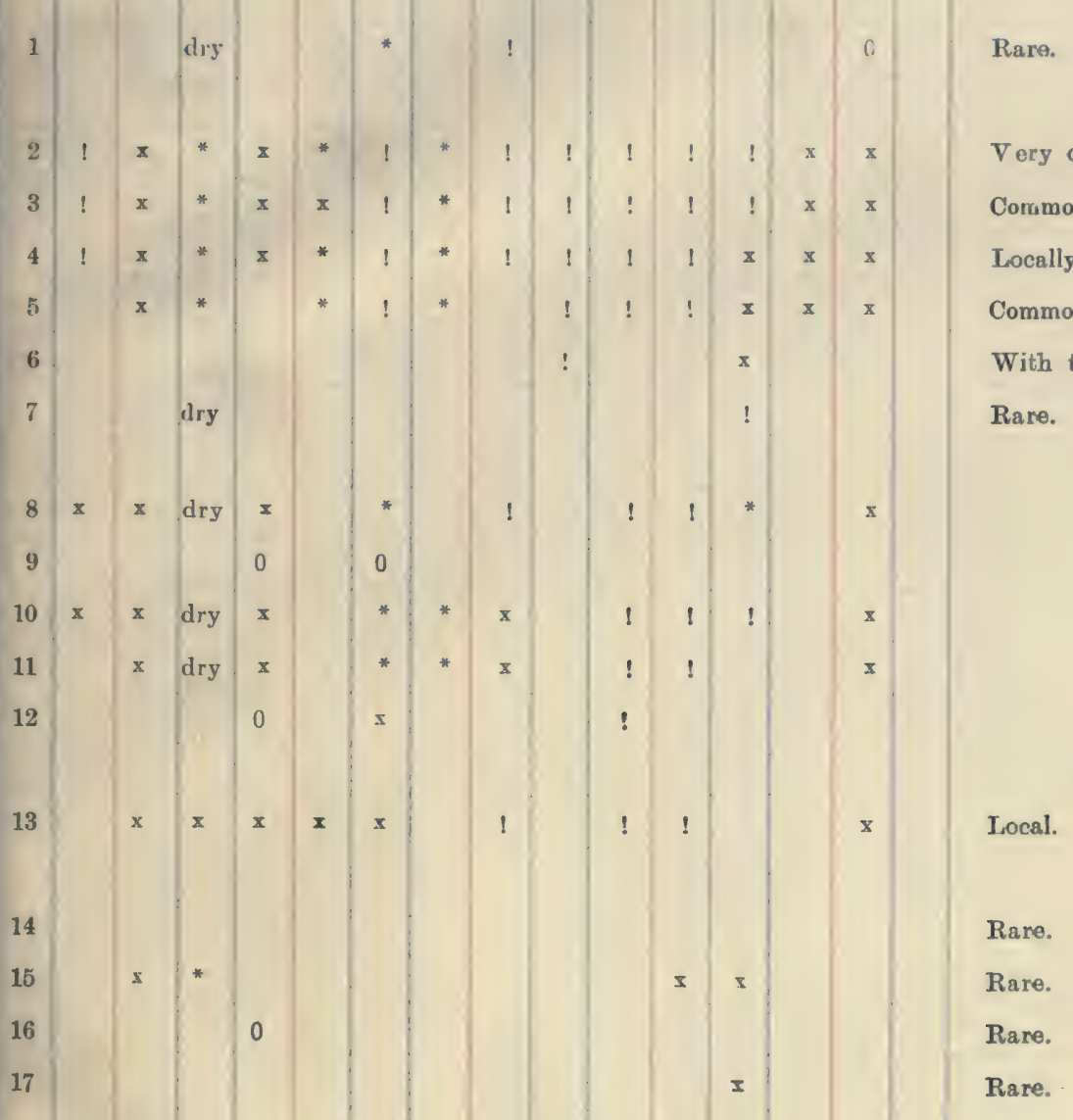







\section{PLEASE DO NOT REMOVE CARDS OR SLIPS FROM THIS POCKET}

\section{UNIVERSITY OF TORONTO LIBRARY}

QK Craib, Villiam Grant

308 * The flora of Banffshire

C8

Biological

\& Medical 
